\title{
Quantitative PET in the 2020s: A Roadmap
}

Steven R Meikle ${ }^{1,2}$, Vesna Sossi ${ }^{3}$, Emilie Roncali ${ }^{4}$, Simon R Cherry ${ }^{4,5}$, Richard Banati ${ }^{1,2,6}$, David Mankoff ${ }^{7}$, Terry Jones ${ }^{5}$, Michelle James ${ }^{8,9}$, Julie Sutcliffe ${ }^{4,10}$, Jinsong Ouyang ${ }^{11}$, Yoann Petibon ${ }^{11}$, Chao Ma $^{11}$, Georges El Fakhri ${ }^{11}$, Suleman Surti ${ }^{12}$, Joel S. Karp ${ }^{12}$, Ramsey D. Badawi ${ }^{4,5}$, Taiga Yamaya ${ }^{13}$, Go Akamatsu $^{13}$, Georg Schramm ${ }^{14}$, Ahmadreza Rezaei ${ }^{14}$, Johan Nuyts ${ }^{14}$, Roger Fulton ${ }^{2,15}$, André Kyme ${ }^{2,16}$, Cristina Lois ${ }^{11}$, Hasan Sari ${ }^{17,18}$, Julie Price ${ }^{17,18}$, Ronald Boellaard ${ }^{19}$, Robert Jeraj ${ }^{20,21}$, Dale L Bailey ${ }^{1,22,23}$, Enid Eslick ${ }^{22}$, Kathy P Willowson ${ }^{22,23}$, Joyita Dutta ${ }^{24}$

1 Sydney School of Health Sciences, Faculty of Medicine and Health, The University of Sydney 2 Brain and Mind Centre, The University of Sydney

3 Department of Physics and Astronomy, University of British Columbia

4 Department of Biomedical Engineering, University of California, Davis

5 Department of Radiology, University of California, Davis

6 Australian Nuclear Science and Technology Organisation

7.Department of Radiology, University of Pennsylvania

8 Department of Radiology, Molecular Imaging Program at Stanford (MIPS), CA, USA

9 Department of Neurology and Neurological Sciences, Stanford University, CA, USA

10 Department of Internal Medicine, University of California, Davis, CA, USA

11 Gordon Center for Medical Imaging, Massachusetts General Hospital and Harvard Medical School

12 Department of Radiology, University of Pennsylvania

13 National Institute of Radiological Sciences (NIRS), National Institutes for Quantum and

Radiological Science and Technology (QST), Chiba, Japan

14 Department of Imaging and Pathology, Nuclear Medicine \& Molecular imaging, KU Leuven

15 Department of Medical Physics, Westmead Hospital

16 School of Biomedical Engineering, Faculty of Engineering and IT, The University of Sydney

17 Department of Radiology, Massachusetts General Hospital \& Harvard Medical School, Boston, MA, USA

18 Athinoula A. Martinos Center, Massachusetts General Hospital \& Harvard Medical School, Boston, MA, USA

19 Radiology and Nuclear Medicine, Cancer Center Amsterdam, Amsterdam University Medical Center, location VUMC

20 Departments of Medical Physics, Human Oncology and Radiology, University of Wisconsin

21 Faculty of Mathematics and Physics, University of Ljubljana

22 Department of Nuclear Medicine, Royal North Shore Hospital

23 Faculty of Science, The University of Sydney

24 Department of Electrical and Computer Engineering, University of Massachusetts Lowell 


\section{Abstract}

Positron emission tomography (PET) plays an increasingly important role in research and clinical applications, catalysed by remarkable technical advances and a growing appreciation of the need for reliable, sensitive biomarkers of human function in health and disease. Over the last 30 years a large amount of the physics and engineering effort in PET has been motivated by the dominant clinical application during that period, oncology. This has led to important developments such as PET/CT, whole-body PET, 3D PET, accelerated statistical image reconstruction, and time-of-flight PET. Despite impressive improvements in image quality as a result of these advances, the emphasis on static, semiquantitative "hot spot" imaging for oncologic applications has meant that the capability of PET for quantifying biologically relevant parameters based on tracer kinetics has not been fully exploited. More recent advances, such as PET/MR and total body PET, have opened up the ability to address a vast range of new research questions from which a future expansion of applications and radiotracers appears highly likely. Many of these new applications and tracers will, at least initially, require quantitative analyses that more fully exploit the exquisite sensitivity of PET and the tracer principle on which it is based. It is also expected that they will require more sophisticated quantitative analysis methods than those that are currently available. At the same time, artificial intelligence is revolutionizing data analysis and impacting the relationship between the statistical quality of the acquired data and the information we can extract from the data. In this roadmap, leaders of the key sub-disciplines of the field identify the challenges and opportunities to be addressed over the next 10 years that will enable PET to realise its full quantitative potential, initially in research laboratories and, ultimately, in clinical practice. 


\section{Introduction: Current and future capabilities of quantitative PET}

Vesna Sossi ${ }^{1}$, Emilie Roncali ${ }^{2}$, Simon R Cherry ${ }^{2,3}$, Steven R Meikle ${ }^{4}$

1 Department of Physics and Astronomy, University of British Columbia

2 Department of Biomedical Engineering, University of California, Davis

3 Department of Radiology, University of California, Davis

4 Sydney School of Health Sciences, The University of Sydney

\section{Status}

Over the past two decades, most commercial PET systems have transitioned from using bismuth germanate (BGO) scintillators coupled to photomultiplier tubes (PMTs) to lutetium-based scintillators read out by silicon photomultipliers (SiPMs). Scintillators based on lutetium silicates are bright, dense and fast, which translates into high efficiency, good energy and timing resolution, and good spatial resolution. SiPMs have rapidly emerged as the optimal photodetector for PET. They are robust, compact, low-cost, have high gains and, importantly, are insensitive to magnetic fields; these characteristics are readily catalysing novel detector designs. The combination of these scintillators with new photodetectors and advances in the speed, flexibility, and density of electronics laid the foundation for the development of time-of-flight PET scanners with timing resolution now approaching 200 ps in the latest generation of commercial systems. This timing resolution allows for the localization of the positron annihilation along the line of response within $3 \mathrm{~cm}$, thus significantly improving the statistical quality of the images and related signal to noise ratio (SNR), which can be utilized to either improve the usable spatial resolution or decrease the injected dose or scanning time, depending on the need.

PET is almost always integrated with CT or, more recently, MRI to provide anatomic context required for correction of attenuation and scattered events. Combinations of PET with other modalities such as ultrasound, EEG and optical imaging (preclinically) are also being developed. Long axial field of view (1-2 metres) PET systems have recently been developed which provide exquisite detection sensitivity $[1,2]$. Such high detection sensitivity can be exploited in numerous ways; excellent quality images can be acquired with a fraction of the traditional injected dose or scanning time; the effective temporal resolution can be improved from minutes to seconds and all organs in the body can be imaged simultaneously, which provides new possibilities for the investigation of multi-organ tracer kinetics, signalling and pathologies.

While hardware development has contributed to a desired combination of improved image quality and dose/scanning time reduction, significant additional gains have been provided by software and algorithm development. 4D, MRI-aided and artificial intelligence (AI) based image reconstruction algorithms have resulted in further gains in SNR, leading to images that were hardly imaginable 10 15 years ago. Similarly, advances in post-reconstruction image analysis, including radiomics-, connectivity- and Al-based methods, enable more subtle biomarkers of human function and disease to be extracted from information rich datasets. Finally, the increased SNR and effective temporal resolution of PET systems is driving renewed interest in tracer kinetic modelling at the voxel level (parametric imaging) and greater recognition of the importance of motion correction [3].

\section{Current and Future Challenges}


There has been a clear contextual shift in our understanding of the complexity of the human body and an increasing appreciation of its many interacting systems in health and disease (see, for example, $[4,5]$ ). The imaging tools of the future must be able to capture this complexity, using multi-modal imaging systems, well-designed radiotracers, and sophisticated quantitative analysis tools that view the entire body as an integrated system.

Imaging and therapy will need to continue along their path of convergence. Theranostics is rapidly evolving from a concept to a real clinical paradigm, perhaps seen nowhere better than in the integration of radionuclide imaging and radionuclide therapy. There is enormous potential for increasing the use of internal targeted radionuclide therapy by expanding the repertoire of targeting molecules for delivering radiation to tumour sites. However, major challenges lie on our doorstep for developing the quantitative tools that are necessary for accurate, reproducible, personalized dosimetry [6]. These tools are at the core of the safe and effective use of external beam radiation therapy. Comparatively, radionuclide therapy planning is in the stone age. Specific challenges include the need for quantification at high spatial resolution to capture the heterogenous dose distribution frequently observed, which will be the foundation of multiscale dosimetry. Quantitative multiscale dosimetry will be essential to fully optimize emerging alpha-emitters (range $\sim 100 \mu \mathrm{m}$ ) for clinical use.

The quantitative methods we develop need to be harmonized and highly reproducible for translation to the clinic. Quantitative PET is an appealing concept but difficult to achieve accurately and reproducibly in practice [7]. Without great care, test-retest studies on the same subject on the same scanner can show large variations, and these are only exacerbated as one moves across different imaging platforms and centres, each with their own methods, protocols and algorithms [8]. What are the metrics beyond the standard uptake value that we can measure reliably and that can be used to implement consistent diagnostic criteria and biomarkers? How reproducible are these numbers, and how much more reproducible can we make them given the biological variability inherent in measuring function using a radiotracer whose distribution changes in time and space? Furthermore, despite impressive advances in quantitative analysis methods, several challenges remain stubbornly in place, especially those associated with non-rigid and non-periodic motion, and non-uniform resolution and noise properties that are not always easily characterized.

Other important issues of a more general nature require our attention as well. PET clearly has an access problem. It is an expensive technology, available only to a privileged minority of the world's population. Cost is a barrier, but not the only one. More effort needs to be devoted to developing the necessary research and clinical infrastructure, training environment and relevant clinical applications (for example infectious diseases) to address the needs of developing countries and remote communities. As well as training the next generation of imaging scientists, we also need to educate non-experts, including the general public, about the safe use of ionizing radiation. While we are making great strides to reduce the radiation dose from medical imaging examinations, it will never be zero. So, while we continue to work on techniques that further reduce dose while maintaining quantitative accuracy, in parallel we also need to focus on education, ensuring all of us in the field are able to explain the doses received from PET studies in the context of risks more familiar to the patient. This takes time and effort, but if we are to be successful in the future, we will need to inform and shape public perceptions about the risks associated with PET and CT examinations. 


\section{Advances in Science and Technology to Meet Challenges}

A number of advances in technology are required to address existing challenges and reach the ultimate performance potential of PET afforded by the underlying physics. Most notably, timing resolutions on the order of 10-20 ps would almost eliminate the need to perform traditional image reconstruction as each positron annihilation event would be recorded with 2-3 mm precision in 3 dimensions. However, current technologies cannot get us there [9]. Scintillators have too slow a rise time, and other mechanisms, such as Cerenkov luminescence, produce only a dozen or so photons. Photodetector technology that can reliably detect such small signals, across $\mathrm{mm}^{2}$ of sensor area, and have a sufficiently fast response time, do not yet exist. As new materials and photodetectors emerge, efficient integration of these detector components including fast and low-noise electronics will become more and more critical to approaching the limits of timing resolution. While 10-20 ps may not be a realistic goal in the near future for clinical systems, 100 ps time resolution may be achievable.

The improvements in SNR achieved with the first long axial field of view PET systems, that collect a much larger fraction of the available signal, have afforded a compelling glimpse of the future $[1,2]$. However, a major barrier to the widespread adoption of these systems is the cost inherent in the volume of detector material and large numbers of electronic channels. Thus, technological advances that can greatly reduce these costs, through new low-cost materials, sensors and electronics, are a key need.

On the computational side, the rapid adoption of deep-learning methods is opening new frontiers, from processing signals as they are collected on the scanner, through image reconstruction, data corrections and artefact removal, and post-processing of images including segmentation and registration [10]. What will be the role of these methods in the future? Where are they best applied? How do we validate them? And, how do we standardize our methods and protocols to ensure their reliable and effective use in clinical and research settings?

\section{Concluding Remarks}

Ultimately, PET should be a quantitative tool that fully exploits the tracer principle, makes optimal use of the administered radiation dose, and works seamlessly with a range of radiotracers to measure a spectrum of physiological processes, metabolic pathways and molecular targets, across the entire human body. It should produce reliable, reproducible metrics that inform accurate clinical decision making and support high quality research and clinical trials outcomes and it should easily integrate with other imaging modalities when additional, complementary, information is needed. It should also be globally accessible as a cost-effective tool in the management of important regional health problems. Despite impressive advances over the past two decades, we are still far from that ideal, yet there is nothing fundamental to prevent us from getting there. This roadmap aims to provide a useful snapshot of where we are now, where we want to be in the future and how we might get there, as well as being a catalyst for debate. 


\section{Applications of quantitative PET in neuroscience}

\section{Richard Banati ${ }^{1,2}$ and Steven R Meikle ${ }^{2}$}

1 Australian Nuclear Science and Technology Organisation

2 Sydney School of Health Sciences, The University of Sydney

\section{Status}

From the earliest studies in the 1970s, PET has been extensively applied in the basic and clinical neurosciences. The self-evident reason is that tracer/ligand-based molecular imaging with PET yielded the first biologically specific information about the neurochemistry of the untouched living brain. The initial focus of PET brain imaging was on the quantitative regional assessment of blood flow, glucose metabolism and oxygen extraction and metabolism, including task-directed activation of transient changes in these parameters - a paradigm that spawned the field of fMRI. Subsequently, investigators turned their attention to enzyme assays (e.g. Fluorodopa and MAO-B) and ligand binding to receptors and protein aggregates to describe the functioning of the brain under normal, activated, and pathological conditions (figure 1) [11].

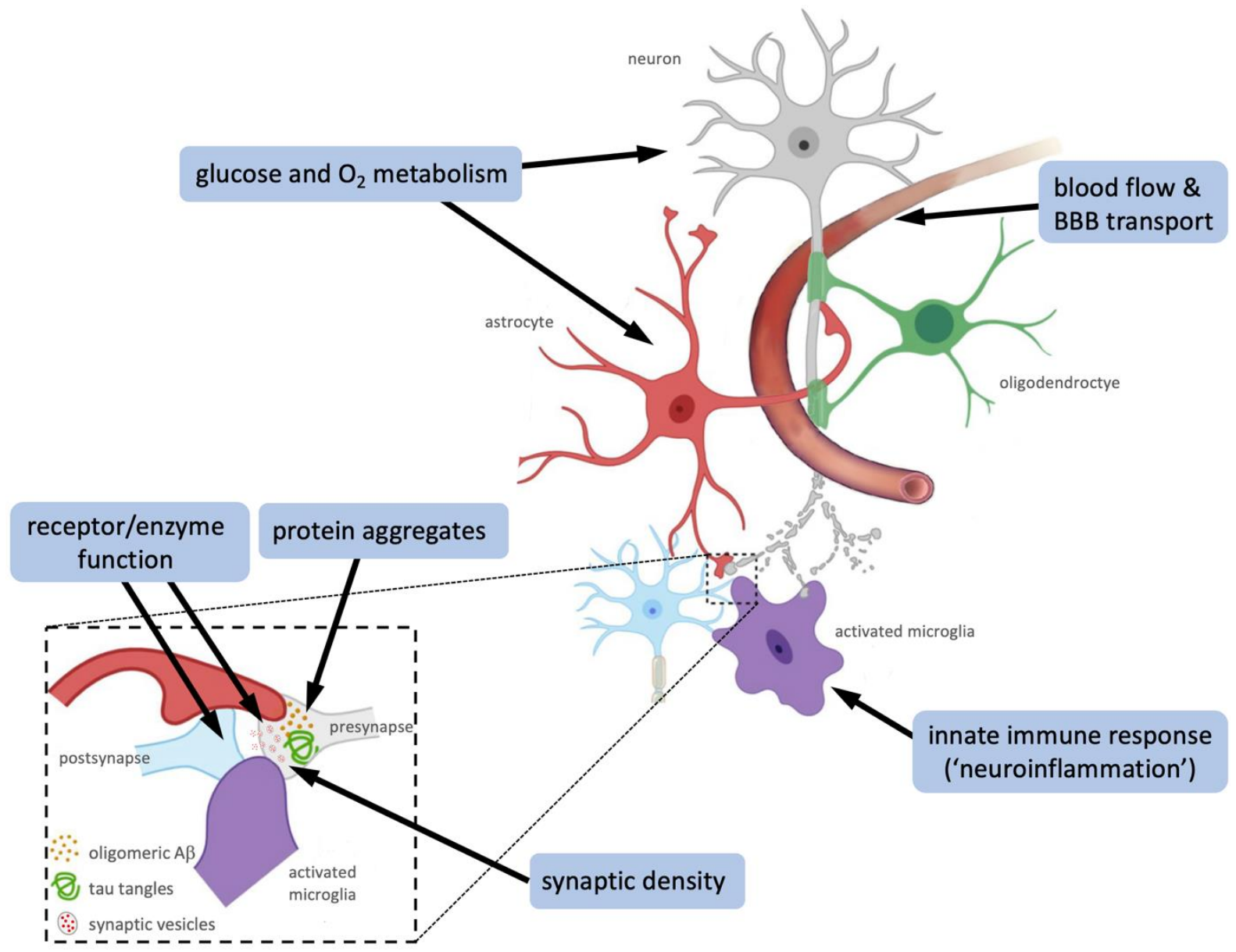

Figure 1. Pictorial overview of the major PET functional domains investigated over the past four decades and cellular sources for the corresponding targets in the brain (based on figure 2 from [12]).

Since the beginning of PET, there has been a steadily rising interest in the detection of active brain tissue pathology, i.e. the neuropathological imaging of acute or chronic diseases of the brain [13]. The paradigm of imaging and quantifying the brain's innate immune response to common pathologies was 
established with a selective ligand for the inducible mitochondrial $18 \mathrm{kDa}$ translocator protein (TSPO) [14]. The TSPO is expressed in low abundance in the normal brain but upregulated, predominantly in the mitochondria of microglia, the brain's immune effector cell, in the wake of progressive tissue pathology. It has subsequently popularised the concept of 'neuroinflammation', now in need of a more refined understanding of non-neuronal changes associated with pathology. This includes better characterisation of the specificity and selectivity of TSPO tracers under in vivo imaging conditions (see, for example [15]) as well as the development of ligands for other binding sites with a functional role within the cellular cross-talk between the nervous and immune systems.

Imaging the accumulations of beta amyloid $(A B)$ plaques and tau-activated neurofibrillary tangles, the hallmarks of Alzheimer's disease (AD), in the brains of dementia patients has been a significant recent focus. The development of PET ligands for $A \beta$ and tau is predicated on the assumption that quantifying the accumulation of these protein aggregations early in the course of the disease may help stratify patients by clinical phenotype, add prognostic value and aid in the monitoring of disease-modifying therapies [16]. While promising, the correlation between $A \beta$ plaques and clinical phenotype may be tenuous, as is the presence of tau pathology [17]. $A \beta$ and tau can be at different stages of aggregation, have differences in turnover rate and are compositionally heterogenous, giving rise to non-specific binding [18]. Whereas earlier ligands for $A \beta$ and tau acted like dyes that intercalate with periodic features of the aggregate, newer ligands appear to behave more like selective receptor ligands yielding the conventional affinity and competitive binding data on which kinetic modelling rests. The value of $A \beta$ and tau quantitative imaging for the neuropathology-based disease staging of dementia may be enhanced when combined with quantitative fluorodeoxyglucose (FDG) PET [19], receptor imaging, or regional blood vessel function [20].

\section{Current and Future Challenges}

With the radiopharmaceuticals, instrumentation and kinetic modelling approaches currently available, PET is well able to detect and quantify pathological processes in the brain with good targetto-background contrast, such as the examples highlighted above. However, many important pathologies and receptor systems have a sparse distribution, giving rise to low contrast and marginal signals. Most notably, receptors on migratory cells such as macrophages, that often critically determine the progression of pathology, tend to be present at low density except in focal lesions. Currently achievable signal-to-noise ratios (SNR) fall below the detection limit of PET in such cases or lead to bias in the estimation of marginal signals and do not yet allow reliable in vivo tracking of migratory cells. This is also likely to be the case for important peripherally expressed targets, such as dopamine receptors and immunocytes, that have so far only been studied with PET in the brain or in highly focal peripheral lesions. Possible solutions to enable quantitative imaging of these more challenging targets include radiopharmaceuticals with optimal imaging characteristics (see section 5), PET scanners with significantly higher sensitivity (section 8 ) and spatial resolution and more sophisticated data analysis methods (sections 10, 12 and 15).

An obvious current impediment to the progress of imaging neuropathology by PET is the severely restricted field of view of most current scanners. This precludes imaging of the entire central nervous system, i.e. brain and spinal cord, let alone the ability to capture the interactions of the peripheral nervous system with other organs of the body (figure 2). These limitations have meant that, until recently, imaging has had little to offer patients suffering from spinal trauma or any of the 
heterogenous group of neuromuscular conditions that includes spinal muscular atrophy (SMA) in children and amyotrophic lateral sclerosis (ALS) in adults. These rapidly progressive neuropathies are broadly characterised by impaired signalling between the CNS and the neuromuscular junction, resulting in profound motor dysfunction and shortened life expectancy [21]. The lack of reliable, sensitive imaging biomarkers to enable the study of so-called "embodied Neurology" [22] means that not only can we not offer these patients early assessment of treatment response, but clinical trials of promising new therapies take longer to achieve conclusive endpoints.
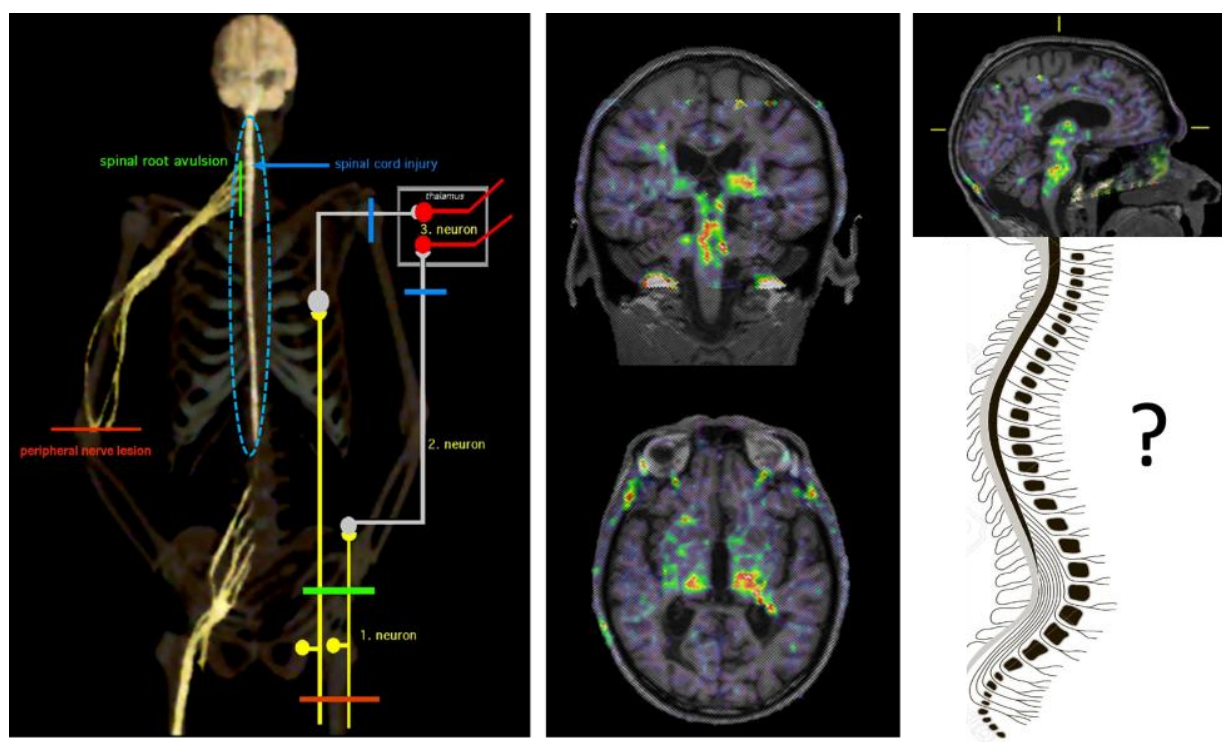

Figure 2. The schematic on the left illustrates how lesions in the spinal cord or peripheral nerves can cause remote expression and imaging signals, here exemplified by the expression of mitochondrial TPSO using $\left.{ }^{11} \mathrm{C}\right](\mathrm{R}) \mathrm{PK} 11195-\mathrm{PET}$. Signals can be expected along the entire tract of the injured first neuron, via the ascending pathways of the second neuron and trans-synaptically at the site of the input-receiving third neuron. $\left.{ }^{11} \mathrm{C}\right](\mathrm{R}) \mathrm{PK} 11195-\mathrm{PET}$ shows a patient with secondary progressive multiple sclerosis in whom all three levels of the neuronal connection between peripheral nerve, spinal cord and brain are expected to be altered by the disease [13]. Indeed, a small stretch of a larger neural pathway is discernible in the thalamus with contralateral continuation through the brainstem into the spinal cord which lies outside the field of view.

Apart from sensitivity constraints and the limited field of view, the spatial resolution of current PET scanners is inadequate to reliably detect and quantify sparse signals in the brain and spinal cord. This hinders a more systematic study of metabolic activity and axonal connectivity, both important for the understanding of spinal disease, injury and recovery [23] as well as the neuromuscular conditions discussed above.

\section{Advances in Science and Technology to Meet Challenges}

Fortunately, recent advances in PET instrumentation and data analysis methods are poised to address many of the significant challenges outlined above. Arguably, the most significant of these is the recent development of long ( $>1$ metre) axial field of view (FoV) PET scanners, that are able to simultaneously image all the major organs of the body with an order of magnitude increase in sensitivity compared with current technology [1]. Together with further gains in SNR due to continuous improvements in time-of-flight (TOF) resolution and spatial resolution better than $3 \mathrm{~mm}$, researchers and clinicians now have access to tools able to capture and quantify signals from diffuse and sparsely distributed pathology, which so far remains the least well understood contributor to clinical disease progression. Total-body PET systems will not only enable the entire CNS to be imaged with very high sensitivity, 
they will also enable non-invasive sampling of the arterial input function for kinetic modelling from the heart and major vessels within the FoV.

Artificial intelligence (AI) is already making a significant contribution to a wide variety of problems in medical imaging. This topic is dealt with in detail in section 15. Here we highlight two areas where Al may help address some of the pressing challenges in PET neuroimaging. One is resolution recovery, or more specifically super-resolution, where the goal is to convert resolution-limited PET images to images consistent with an intrinsically higher spatial resolution system [24]. This may be particularly useful, not only in the brain where resolving the individual cortical layers is beyond the limits of current PET scanners, but also for imaging the spine where the signals of interest challenge the detection and quantitative capabilities of current PET systems. Another useful role for Al is in denoising PET data in either the spatial or temporal domains or both. Even with the substantially higher SNR of long axial FoV PET systems with good TOF resolution, the noise levels in voxel-based time-activity curves remain relatively high, especially for signals arising from sparsely distributed targets such as peripheral receptors or transient neurotransmitter signals. Al-based denoising techniques can be expected to improve the accuracy and reliability of parameter estimates, including parametric images.

Finally, if PET is to realise its full potential for imaging the entire CNS and bi-directional signalling with other organs, methods to correct for not only head motion but also complex organ movements will be essential (see section 11). For example, the ability to image the gut-brain axis, including normal and abnormal gut barrier functions and interactions with the CNS, would open new avenues for studying neurodegenerative conditions, such as Parkinson's disease, where there is an increasing appreciation for the role of immune signalling from the intestines [4]. The ability to measure and account for nonrigid motion of the torso and internal motion due to gut motility will be critical to the success of PET in such studies.

\section{Concluding Remarks}

In summary, high resolution, high sensitivity imaging of the entire nervous system as an integral component of the complex human organism promises an exciting future for quantitative PET neuroimaging over the next decade. While further challenges remain in optimising the use of PET in the brain and especially in establishing its clinical role in the management of $A D$ and other forms of dementia, along with mood disorders, psychotic disorders and substance abuse, we see opportunities in focussing more on the distributed hormonal and immune systems to which the nervous system is so intimately connected and reciprocally regulated. Such a systems biology approach will help the brain and behavioural sciences overcome some of their 'disembodied' theorising about the brain and mind and enable a better understanding of how the plasticity of other organ systems intersects with the plasticity of the brain.

\section{Acknowledgements}

The authors acknowledge the continual support of the Australian Nuclear Science and Technology Organisation (ANSTO), as well as research funding from the Australian Institute of Nuclear Science and Engineering, the Australian Research Council, the National Health and Medical Research Council and the National Imaging Facility. 


\section{Applications of Quantitative PET in Cancer David Mankoff \\ Department of Radiology, University of Pennsylvania}

\section{Status}

For cancer, $\mathrm{PET/CT} \mathrm{has} \mathrm{been} \mathrm{used} \mathrm{largely} \mathrm{for} \mathrm{staging} \mathrm{and} \mathrm{detection,} \mathrm{relying} \mathrm{heavily} \mathrm{on} \mathrm{qualitative}$ interpretation. However, the emerging use of PET/CT to guide therapy has increased interest in quantitative PET cancer imaging. One important application of quantitative PET to cancer is as a molecular imaging cancer biomarker. Choosing individualized cancer therapy on the basis of the patient characteristics and tumour biologic features - often termed precision oncology - is increasingly the goal of care for cancer treatment [25]. Therapy choices in precision oncology are guided by cancer biomarkers that provide information on tumour biologic features that can predict clinical behaviour, guide treatment choices, and assess therapeutic response. (see table 1). Cancer biomarkers are usually measured by assay of tissue samples; however, PET molecular imaging is emerging as a potentially impactful and non-invasive cancer biomarker, including use as a surrogate endpoint in clinical trials for some diseases such as lymphoma [26]. Quantification of tracer uptake in the tumour has been an essential component of PET's use for cancer biomarkers [27].

Table 1 - Description of the types of cancer biomarkers used to direct cancer treatment.

\begin{tabular}{|c|l|}
\hline Biomarker & \multicolumn{1}{c|}{ Description } \\
\hline Prognosis & $\begin{array}{l}\text { Predicts likelihood of death or other adverse outcome; related to features of the } \\
\text { cancer independent of approach to treatment }\end{array}$ \\
\hline Prediction & Predicts likelihood of response to a specific treatment \\
\hline Response & $\begin{array}{l}\text { Assesses whether or not the patient has responded to the treatment, often described } \\
\text { in categories of progression, stable disease, partial response, complete response }\end{array}$ \\
\hline Surrogate Endpoint & $\begin{array}{l}\text { Response measure highly predictive of important downstream patient outcomes } \\
\text { such as overall survival or disease-free survival }\end{array}$ \\
\hline
\end{tabular}

A second need for quantitative PET for cancer relates to the use of PET imaging as a companion diagnostic for radionuclide therapy, often termed as theranostics, both for treatment selection and for estimation of cancer and normal tissue radiation dosimetry [28]. Several new theranostic agents have been approved in a number of countries worldwide in the past few years, and more are coming. While companion diagnostic imaging and dosimetry calculations have traditionally used SPECT, PET has gained increasing favour in this role due to its improved sensitivity and quantitative accuracy, as well as the more widespread availability of longer-lived positron-emitting isotopes for PET imaging (e.g., ${ }^{124}$ I and positron-emitting radiometals) that are suitable for dosimetry studies for longer-liver therapeutic nuclei (e.g., ${ }^{131}$ I and beta-emitting radiometals).

\section{Current and Future Challenges}

PET biomarkers are attractive tools for guiding cancer treatment in clinical trials and clinical practice [26]. PET cancer biomarkers require quantitative imaging approaches that are practical, clinically robust, well defined, reproducible, and validated as cancer biomarkers in rigorous clinical trials. Response biomarkers are the most common type of PET biomarker in current clinical practice, and some markers have also become surrogate endpoints. The most well-developed PET response measure is for lymphoma; however, this application uses mostly qualitative interpretation criteria. Other response applications, however - for example, serial FDG PET/CT to evaluate breast cancer bone 
metastasis response to systemic therapy (figure 1) - have used more quantitative approaches. For the bone metastasis example, quantitative changes in uptake predict key downstream clinical events such as disease progression and skeletal-related events [29]. Emerging criteria for quantitative response, akin to criteria used for size-based response measures (e.g., RECIST), are gaining favour. The PERCIST criteria [30], for example, performed well in application to bone metastasis response assessment and is an emerging standard. Beyond static imaging measures like SUV, parameters obtained from dynamic (4D) PET images may offer increased biologic insight and predictive value. For example, FDG delivery $\left(\mathrm{K}_{1}\right)$ and glycolytic flux $\left(\mathrm{K}_{\mathrm{i}}\right)$ constants significantly outperformed SUV in their ability to predict important breast cancer treatment outcomes such as pathologic response and disease-free survival [31].
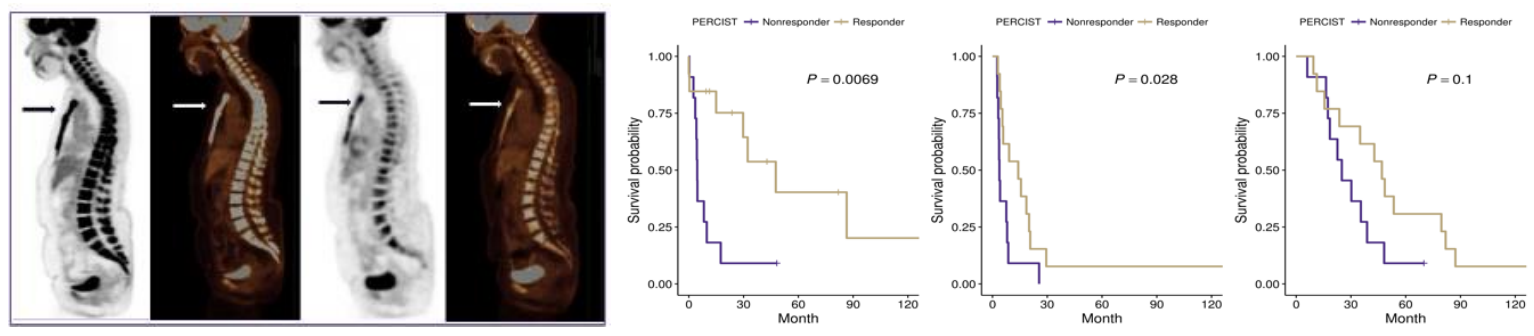

Figure 1. (Left panel) Serial FDG PET/CT in a patient with diffuse bone metastases shows a dramatic decrease in FDG uptake in response to endocrine therapy. (Right panel) Measures of the change in FDG uptake with therapy, quantified and categorized according to PERCIST criteria, predict Progression Free Survival (left) and Time to Skeletal Related Event (middle), with a trend for predicting Overall Survival (right) in a group of patients undergoing treatment for metastatic breast cancer. (Right panel taken from [26]).

The use of PET as a prognostic and/or predictive cancer biomarker relies on absolute quantitative uptake measures to define thresholds for predicting outcomes. For example, studies using $\left[{ }^{18} \mathrm{~F}\right]$ fluoroestradiol (FES) PET to image breast cancer have shown that SUV $=1.5$ provides a threshold for determining which tumours express sufficient estrogen-receptor (ER) for endocrine therapy, below which response is unlikely [32] (figure 2). This marker could be used to help choose treatment for patients with metastatic disease from ER+ breast cancer, where biopsy of multiple sites poses a challenge. Consistent, precise, and unbiased estimates of tracer uptake will be important for application to clinical practice for FES and other predictive PET imaging biomarkers.

Radiopharmaceutical radiation dosimetry is an important and widely used approach for diagnostic and therapeutic radiopharmaceutical testing and drug approval and is also used clinically to guide therapy in some selected uses such as $\mathrm{Na}^{131}$ | for metastatic thyroid cancer and [ ${ }^{131} \mid$ ] $\mathrm{mIBG}$ for neuroendocrine tumours. Thus far, radiopharmaceutical radiation dosimetry has been used largely to assess normal tissue radiation exposure to guide dosing limits. Current methods that rely heavily on single-photon planar imaging methods cannot accurately estimate retention of therapeutic radiopharmaceuticals at tumour sites, a critical component for assuring tumour response, akin to the approach used in external beam radiotherapy. The use of $\mathrm{Na}^{124}$ I PET/CT to estimate $\mathrm{Na}^{131}$ I radiation dosimetry, for example, has been shown to provide estimates of both normal tissue and tumour radiation dose, with the potential to guide more rational and effective treatment of metastatic thyroid cancer [33] and other cancers. Improvements in PET image quality and data accuracy for low levels of activity, a key component of accurate dosimetry, will increasingly support quantitative PET imaging as a tool for therapeutic radiopharmaceuticals. 


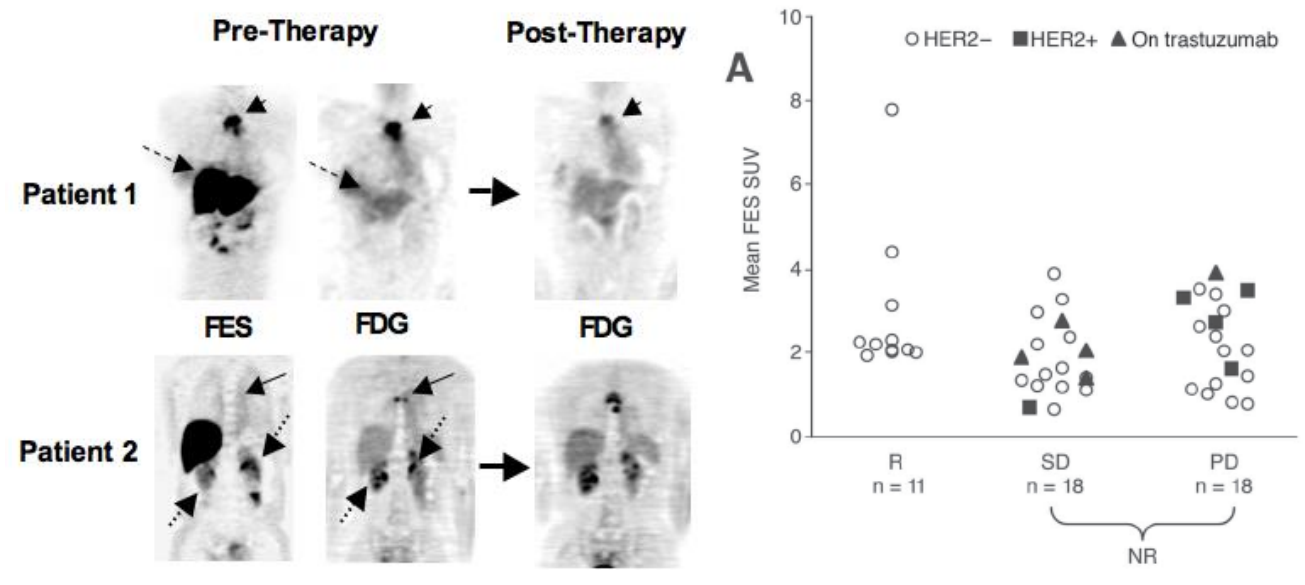

Figure 2. (Left panel) Coronal PET images of ${ }^{18}$ F-fluoroestradiol (FES) uptake (left) and FDG uptake before (middle) and after (right) endocrine therapy are shown for two patients with metastatic disease from estrogen receptor positive (ER+) breast cancers. Patient 1 showed metastases (solid arrow) that were metabolically active by FDG PET with matched uptake of FES, indicating preserved ER expression. Patient 2 showed a site of bone metastasis by FDG PET (solid arrow) but no corresponding uptake by FES, suggesting a loss of ER expression. Patient 1 had an excellent objective response while Patient 2 had disease progression, as indicated by changes in the post therapy FDG scans. Normal liver (dashed arrows) and kidney uptake (dotted arrows) is also seen in the images for both radiopharmaceuticals. (Right panel) Quantitative analysis of FES PET showed that no patient with tumour SUV $<1.5$, indicative of loss of ER expression, had an objective response $(R)$ to endocrine therapy. (adapted from [30]).

\section{Advances in Science and Technology to Meet Challenges}

Advances in the science and technology needed to meet the challenges of quantitative PET for cancer imaging applications fall into several categories:

(1) Advances in PET instrumentation - Improvements in imaging tomograph sensitivity, quantitative accuracy, and $4 \mathrm{D}$ imaging capabilities will provide the dynamic operating range needed for both biomarkers and dosimetry, including fast whole-body coverage, dynamic 4D PET imaging, and the ability to generate highly accurate imaging data from small amounts of tracer. More sensitive devices may permit imaging more than one PET tracer in a single session to better characterize tumour phenotype, as has been done in prior breast cancer studies $[29,34]$ by using the low-dose/high-dose paradigm employed in nuclear cardiology, for example. Detector technology advances that include "digital PET", improved time of flight resolution, and large axial field-of-view imaging devices are ideally suited to support quantitative PET cancer imaging [35].

(2) New diagnostic and therapeutic radiopharmaceuticals targeted to cancer-specific targets - The discovery of new molecular targets for cancer drives precision oncology - and the need for new molecular imaging biomarkers - as well as new radionuclide theranostics. The development of novel diagnostic and therapeutic radiopharmaceuticals matched to cancerspecific targets will fuel an expansion of both diagnostic and therapeutic nuclear oncology and drive the need for quantitative PET methods. Recent approvals of novel diagnostic and therapeutic radiopharmaceuticals provide a leading indicator of this trend. 
(3) Automated, reproducible analysis of PET images - From the viewpoint of consistency and clinical practicality, automated image analysis tools facilitate the routine use of quantitative PET imaging metrics in clinical practice. Much of the current quantitative work in the clinic is done manually, introducing operator variability and potential for errors in reporting. Tools to make the process more efficient and robust will increase use and reliability of quantitative PET for cancer, and enable the ability to measure tumour features of importance in application to directing cancer treatment - for example tumour heterogeneity.

(4) Novel 3D and $4 D$ approaches to PET image analysis, including radiomics and artificial intelligence methods - Advances in data science have given rise to the field of radiomics (see section 15), specifically image analysis to identify imaging features beyond what the human observer can usually see. Radiomic features can add diagnostic performance or predictive value to standard quantitative approaches and can also infer regional biologic features such as gene expression. This methodology has been most widely applied to structural images such as CT [36], but can also be applied to PET imaging. Improved PET image quality and quantitative accuracy will increase what can be gleaned from radiomics and other data science tools.

(5) Consensus guidelines for quantitative PET image acquisition, generation, and analysis Beyond technical advances, there is a need for consistent quantitative PET image generation and analysis essential for clinical implementation (see section 13). This requires consensus between scientists, clinicians (both imaging and cancer), manufacturers, and regulators on standard approaches for image acquisition and analysis. Progress in bringing these groups together is seen in Europe (European Imaging Biomarker Alliance, EIBALL)) and America (Quantitative Imaging Biomarkers Alliance, QIBA), along with quantitative imaging-focused research groups such as the US National Cancer Institute Quantitative Imaging Network (QIN).

\section{Concluding Remarks}

Precision oncology and increased use of therapeutic radiopharmaceuticals are rapidly emerging trends in oncology that will drive a need for quantitative PET cancer imaging. Improved instrumentation, new radiopharmaceuticals, novel image analysis methods can fuel these advances. Clinical implementation and impact on patient cancer will require collaboration and consensus on approaches between imaging scientists, imaging clinicians, and cancer physicians.

\section{Acknowledgements}

Dr. Mankoff's efforts in cancer imaging are supported in part by NIH Grants P30CA016520, R33CA225310, U01CA190254 and a Susan G Komen Leadership Grant (SAC130060). 


\section{Emerging applications and grand challenges}

\section{Terry Jones}

Department of Radiology, University of California, Davis

\section{Status}

\section{A brief history}

Advances have been made in PET scanner technology [37], image reconstruction and PET data analyses for forming quantitative functional images. There have been systematic advances in PET scanner sensitivity, principally through 3D data collection [37]. This has made it possible to record and reconstruct images with increased spatial resolution and hence with enhanced quality and quantification of smaller biological structures. These activities have been complimented by appreciable developments in the radiochemistry for radiolabelling tracer molecules along with preclinical programs to develop and characterize new imaging biomarkers for PET applications.

\section{The Status of emerging applications}

Applications of PET to date have been mainly in neurology, psychiatry, oncology, cardiology and to support drug development. Examples of emerging applications for brain disorders are imaging of amyloid and tau, synapses, neuro receptor systems, activated microglia in dementia, and early detection of Parkinson's Disease. In cardiovascular disease, the imaging of activated atheromatous plaques within the coronary arteries and peripheral circulation is attracting attention as is the observation of an association between myocardial ischemia/infarction and cerebral activation. In oncology, imaging biomarkers for early detection and therapeutic response assessment continue to be developed at the pre-clinical level. The use of the long lived 78.4minute half-life Zirconium-89 to label therapeutic antibodies enables their dosimetry to be optimally measured in metastases days after their administration. Specific PSMA imaging agents for metastatic prostate cancer provide the quantitative dosimetry for introducing theranostic agents using longer lived beta emitting radionuclides such as lutetium-177 to label the PSMA imaging agent. The use of "checkpoint inhibitors" for immunotherapy has attracted PET imaging of programmed cell death protein-1/ligand1 (PD-1/PD-L1) blockade and the presence of activated "killer Cell" T lymphocytes within the tumour (Figure 1). It is projected that imaging of activated T cells will find application in other areas of medicine particularly in inflammation and infection, e.g. COVID-19. Pharmaceutical companies continue to use PET to support the development of new pharmaceuticals through pharmacokinetic and pharmacodynamic studies during the early phases of a drug's development. The translation of clinical research findings to healthcare has principally been on diagnosis, staging and treatment assessment for cancer and dementia. There is much current enthusiastic expectation for the use of PET to support theranostics based treatment of cancer.

\section{Why is the field still important?}

From the emerging applications of PET within certain areas of mainstream clinical medicine, it is evident that this field is destined to become ever more important. They point to the future evolution of Total Body PET (TBP) imaging and beyond that of current applications.

\section{What will be gained with further advances?}

Further advances will reinforce the success of the emerging and future applications by improving the practicality, sensitivity, quantitative accuracy and image quality for recording distributions of positron 
emitting imaging biomarkers. This is predicted to produce transformative methodology and scientific impact that will translate into improvements for ongoing experimental medicine and healthcare.

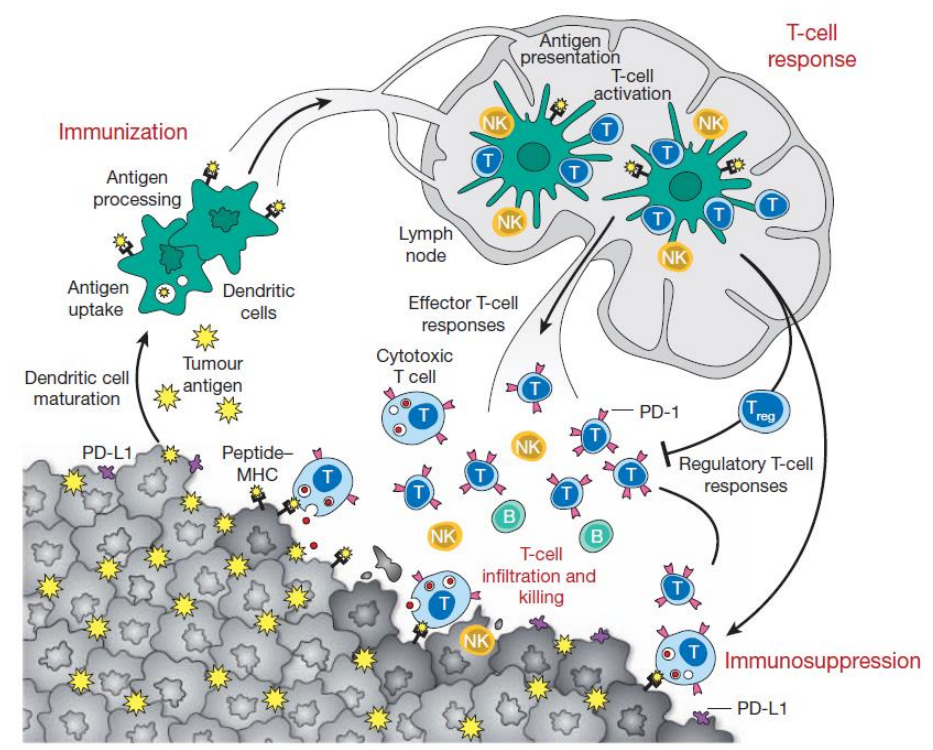

Figure 1: Generation and regulation of anti-tumour immunity-showing T-cell activation. From [38].

\section{Current and Future Challenges}

\section{Big research issues}

These are to:

- Enhance the statistical quality of derived total body quantitative, functional parametric images through 3D data collection, optimal time of flight recording, reconstructions, kinetic modelling and signal averaging.

- These parametric images need to be in the form of metric units of biological processes that are comprehendible to the scientific community including clinical researchers and healthcare practitioners. Such quantitative based forms of communication, as opposed to images of tracer uptake, offer a seminal currency for engaging communities, especially for those not expert in PET methodology. It provides for multidisciplinary appreciation and analysis of data, discussions within respective peer groups, leading to consensus acceptance of the data and formulating on-going research questions and paradigms.

- Make quantification of entities of regional tissue function more readily translatable to healthcare

- Capitalise on enhanced sensitivity to use lower levels of tracer as supplied from distant distribution centres

- Effect translation of the transformative clinical research procedures and findings to healthcare procedures

- Pioneer quantitative ultra-low radiation dose PET studies in radiation sensitive human subjects

- Develop the means to reduce the cost of total body and dedicated brain PET scanners

\section{Big challenges}

To reach the tipping point of demonstrating the unique applications and effectiveness of TBP clinical research and healthcare.

\section{Clinical Research:}

- To derive proofs of concept of the new applications that will be transformative with quantitative TBP scanning. These are destined to be based upon: 
- Detecting occult, low density, multi-system disease such as cancer metastases, inflammation, infection, atheromatous plaques and proteinopathies e.g. amyloid, tau, and alpha-synuclein

- Providing total body, kinetic model based, parametric images for drug delivery and retention, toxicology, theranostics dosimetry of tumours and normal tissues, along with the development of radio-labelled imaging biomarkers.

- Enabling low radiation dose procedures such as repeat, multi-parameter, normal subjects, young patients, maternal-foetal studies, and the use of low doses of long lived, low abundance positron emitting radionuclides.

- Studying the body's cardiovascular system, such as the distribution of tissue blood flow and challenges (activations), and total body distribution of vascular disease including atheromatous plaques.

- To study the "Systems Biology" of human beings. Such studies are destined to cover: pharmacokinetics, pharmacodynamics, oncology, cardiovascular, endocrinology, immunology, infectious diseases, maternal-foetal studies, and brain-body, "The Connectome" interactions. This will require pioneering TBP based simultaneous recording, e.g. of the Hypothalamus Pituitary Adrenal axis in anxiety and depression, brain and inflammatory lesions in the periphery, the serotonin system in the gut and brain, brain-peripheral body synaptic connections, the release of leptin-like molecules from adipose tissue and their targeting in the brain in metabolic syndrome, the passage of nutrients from the mother to the foetus and heart-brain interaction in ischemic heart disease.

- Developing quantitative statistical parametric mapping [39] for identifying across body focal changes in specific organs and tissues.

- To demonstrate the value of achieving increased spatial resolution in brain PET

Clinical Healthcare:

- To translate impacting high spatial resolution brain PET to healthcare

- To translate impacting TBP based clinical research and associated methodology to healthcare

- To develop the use of lower cost detector technology for TBP

- To demonstrate the cost effectiveness of TBP technology and procedures in healthcare:

- Significantly improved image quality

- Widening the healthcare applications of PET as translated from TBP based clinical research

- Scan times of minutes: more patient throughput per unit of time

- Scan times of minutes: less movement blurring

- Minimise the need for arterial blood sampling to generate quantitative parametric images

- Provide total body normal tissue and tumour dosimetry of theranostic agents

- Undertake the clinical load of 3-4 conventional PET scanners, saving on space and staff

- Prescribe PET scans with lower radiation absorbed dose to patients and for screening

- Longer shelf life of radiotracers from distribution centres - minimise in-house GMP costs

\section{Advances in Science and Technology to Meet Challenges}

\section{Technology}

The first two generations of Total Body PET scanners have been developed $[1,2,40]$ and initial normal human scans have been published [1]. The opportunities for improving the performance of a dedicated brain PET have been reviewed [41]. To further enhance the inherent detection sensitivity, efforts are focusing on developing improved coincidence timing to effect better time of flight resolution with the goal of some 10 picoseconds [42]. 
The creation of total body quantitative functional parametric images has been demonstrated from kinetic ${ }^{18} \mathrm{FDG}$ studies [43] using lower noise kernel based reconstructions [44]. Given the highly repetitive voxel time courses in kinetic data sets, the opportunity exists to cluster and through signal averaging increase signal to noise [45].

\section{Science}

The needed scientific advances rest on undertaking proofs of concept clinical research studies to demonstrate that the recently established TBP scanner technology can produce transformative research within the areas identified. This will require the multidisciplinary engagement with clinical scientists to formulate paradigms to undertake the exploratory pilot studies. Collectively this is needed to demonstrate the envisaged paradigm shift for quantitative molecular imaging in humans that Total Body PET is destined to produce.

The next generation of dedicated brain PET scanners will provide higher effective spatial resolution, hence offering quantification of smaller brain structures than is currently possible. Given the current trend for molecular imaging of global brain pathology, scientific questions need to be formulated that exploit the higher spatial precision of the new scanners for molecular imaging of the human brain.

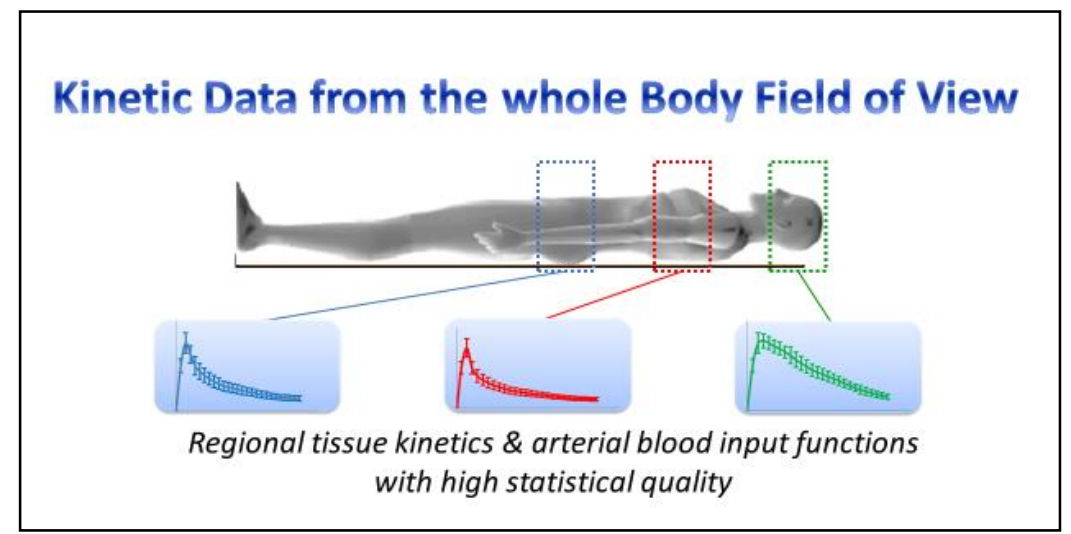

Figure 2: Illustrates the opportunity Total Body PET provides for simultaneously recording whole body regional kinetics of an administered tracer. This includes the means to non-invasively record high quality arterial input functions from within the aorta used to derive kinetic modelling based, whole body parametric functional images.

\section{Concluding Remarks}

The Grand Challenge is to establish the next generation of high spatial resolution brain PET and Total Body PET as the standard means for undertaking PET based molecular imaging in clinical research and healthcare. 


\section{Radiopharmaceuticals for quantitative PET}

Michelle James ${ }^{1,2}$, Julie Sutcliffe $\mathrm{e}^{3,4}$

1 Department of Radiology, Molecular Imaging program at Stanford (MIPS), CA, USA

2 Department of Neurology and Neurological Sciences, Stanford University, CA, USA

3 Department of Internal Medicine, University of California, Davis, CA, USA

4 Department of Biomedical Engineering, University of California, Davis, CA, USA

\section{Status}

Since the introduction of 2-[ $\left.{ }^{18} \mathrm{~F}\right]$ fluoro-2-deoxy-D-glucose ( $\left[{ }^{18} \mathrm{~F}\right] \mathrm{FDG}$ ) over forty-five years ago, numerous PET radiopharmaceuticals have been developed for various applications within the areas of oncology, cardiology, immunology, and neurology, as mentioned in previous sections of this roadmap. The design, synthesis, and successful translation of novel radiolabelled probes for biomarkers of interest is an important undertaking that not only enables new biology to be discovered, but also allows for more accurate detection and effective treatment of different diseases.

The most common radionuclides used for generating PET radiopharmaceuticals are summarized in Table 1. Small molecules are typically labelled with carbon-11 or fluorine-18. Whereas larger molecules, including peptides, antibodies, engineered fragments, adeno-associated viruses, and cells, are most often labelled with gallium-68, fluorine-18, copper-64, zirconium-89, or iodine-124, depending on the biological half-life of the probe of interest. Apart from $\left[{ }^{18} \mathrm{~F}\right] \mathrm{FDG}$, there are several PET radiotracers that are beginning to have a sizeable impact on the way we diagnose and manage various diseases, including prostate cancer [46], neuroendocrine tumours [47], and Alzheimer's disease (AD) [48].

Over the last two decades significant efforts have been made to develop imaging agents to target prostate cancer [46]. Both antibody and small molecules such as quinolone and pyridine have been leveraged - e.g., J591 and PSMA-617. Other PET tracers, including radiolabelled choline, fluciclovine $\left(\left[{ }^{18} \mathrm{~F}\right]-\mathrm{FACBC}\right)$, and probes for prostate-specific membrane antigen (PSMA) have been generated and are currently being evaluated in the clinical setting. Each of these radiotracers has differing mechanisms and excretion profiles which ultimately have an impact on their overall utility as diagnostic agents. In addition to radiotracers being used for detection and monitoring of disease and response to therapies, there has been an explosion of radiopharmaceuticals being developed as theranostic agents, mainly for treating prostate cancer at present [49]. Many of the key isotopes being used for these theranostic agents, such as Lutetium-177, are imaged with SPECT. However, the positron-emitting Copper-64 is often paired with Copper-67 for theranostic applications, while Yttrium-90 emissions can be exploited by both PET and SPECT. (Table 1).

With respect to neuroimaging, advances in the development of imaging biomarkers for $A D$ are changing the way clinical trials are designed and conducted. Specifically, amyloid PET radiotracers, such as $\left.{ }^{18} \mathrm{~F}\right]$ Florbetapir (Amyvid) and $\left[{ }^{18} \mathrm{~F}\right] \mathrm{Flutemetamol}$ (Vizymal), are being used to determine whether participants are eligible for anti-amyloid therapies, and also as a means to assess efficacy of novel treatments [50]. Prior to this type of approach, many participants were included in trials that did not contain the pathology being targeted by the therapy in question, thus wasting valuable resources and generating confusing findings. More recently, tau PET agents have been developed, including $\left[{ }^{18} \mathrm{~F}\right] A V 1451,\left[{ }^{18} \mathrm{~F}\right] \mathrm{PI} 2620$, and $\left[{ }^{18} \mathrm{~F}\right] \mathrm{MK} 6240$, which will hopefully lead to more accurate 
diagnosis and staging of $A D$ (in addition to prediction of treatment response) since tau pathology is more closely associated with cognitive decline [18].

Much growth is expected and greatly anticipated in the areas of theranostics and AD imaging, in addition to the development and translation of new PET radiopharmaceuticals for improving understanding and diagnosis of elusive mental illnesses (e.g., depression) and other neurological diseases for which there remain no accurate methods to confirm diagnosis until post-mortem (e.g., Parkinson's disease).

Table 1. Commonly used radionuclides for generating diagnostic and theranostic radiotracers.

\begin{tabular}{|l|l|l|l|l|l|}
\hline Radionuclide & Half-life & Decay type & $\begin{array}{l}\text { Diagnostic/ } \\
\text { Therapy }\end{array}$ & Production route & Type of Chemistry \\
\hline Carbon-11 & $\begin{array}{l}20.4 \\
\min \end{array}$ & $\beta^{+}(100 \%)$ & Diagnostic & Cyclotron & $\begin{array}{l}\text { Fast } \\
\text { chemistry }\end{array}$ \\
\hline Fluorine-18 & $\begin{array}{l}109.8 \\
\min \end{array}$ & $\begin{array}{l}\beta^{+}(97 \%) \\
\mathrm{EC}(3 \%)\end{array}$ & Diagnostic & Cyclotron & $\begin{array}{l}\text { Fast } \\
\text { chemistry }\end{array}$ \\
\hline Gallium-68 & $68 \mathrm{~min}$ & $\begin{array}{l}\beta^{+}(89 \%) \\
\mathrm{EC}(11 \%)\end{array}$ & Diagnostic & Cyclotron/Generator & Chelation chemistry \\
\hline Copper-64 & $12.7 \mathrm{~h}$ & $\beta^{+}(20 \%)$ & Diagnostic & Cyclotron & Chelation chemistry \\
\hline Zirconium-89 & $78.4 \mathrm{~h}$ & $\begin{array}{l}\beta^{+}(23 \%) \\
\mathrm{EC}(77 \%)\end{array}$ & Diagnostic & Cyclotron & Chelation chemistry \\
\hline Yttrium-90 & $64.6 \mathrm{~h}$ & $\begin{array}{l}\beta^{-}(99.98 \%) \\
\beta^{+}(0.0003 \%)\end{array}$ & Therapy & Cyclotron & Chelation chemistry \\
\hline lodine-124 & $100.2 \mathrm{~h}$ & $\begin{array}{l}\beta^{+}(23 \%) \\
\mathrm{EC}(77 \%)\end{array}$ & $\begin{array}{l}\text { Therapy } \\
\text { Diagnostic }\end{array}$ & $\begin{array}{l}\text { Cyclotron/nuclear } \\
\text { reactor }\end{array}$ & Organic chemistry \\
\hline $\begin{array}{l}\text { Lutetium- } \\
177\end{array}$ & $160.8 \mathrm{~h}$ & $\beta^{-}$ & Therapy & Nuclear reactor & Chelation chemistry \\
\hline Actinium-225 & $238.1 \mathrm{~h}$ & $\alpha$ & Therapy & $\begin{array}{l}\text { Cyclotron/accelerator/ } \\
\text { reactor }\end{array}$ & Chelation chemistry \\
\hline
\end{tabular}

\section{Current and Future Challenges}

Although many different types of molecules can now be successfully radiolabelled for use as PET imaging agents, many challenges remain. These include finding ways to better streamline the identification and validation of suitable biomarkers, increasing education efforts to ensure a consistent pipeline of fully trained radiochemists, and solving specific radiosynthetic issues for labelling small and large molecules. Additionally, there is a need to devise more rapid and costeffective approaches for assessing and translating radiotracers to the clinic for ultimate reimbursement from healthcare providers.

\section{Target discovery and validation}

- High throughput strategies are needed for rapidly identifying and validating promising biomarkers for PET imaging prior to expensive and time-consuming radiochemistry efforts.

- As the field becomes interested in more complex targets - i.e., those with lower concentration of available binding sites ( $B_{\max }$ value) such as alpha-synuclein, or targets with different mutations and/or isoforms (e.g., $3 R$ and $4 R$ tau) - we need to form closer collaborations with structural biologists and synthetic organic chemists to develop radiotracers that meet the required specifications (i.e., higher specificity and affinity).

- To assess suitability of a given target/biomarker of interest, increased access to human postmortem tissue is needed (both healthy control and relevant disease tissue at different stages of severity). 
Radiochemistry methods

- Synthetic approaches to label polyfluorinated groups (e.g. - $\mathrm{CF}_{3}$ ) with high molar activities and non-aromatics with ${ }^{18} \mathrm{~F}$-fluoride, in addition to reliable $\mathrm{C}-\mathrm{H}$ and $\mathrm{C}-\mathrm{OH}$ radiofluorination methods, remain challenging.

- Infrastructure required for carbon-11 labelling of basic functional groups (including ${ }^{11} \mathrm{C}$ labeled amides) continue to be costly and complicated.

- Peptides and larger engineered molecular probes, with ultra-high specificity for a target of interest, have not yet been fully explored for all imaging applications due to challenges regarding tissue (e.g., brain) penetration and clearance.

- Simplification of chemistries to expedite commercialization are needed - i.e., more shakeand-bake strategies and kit-based approaches for radiolabelling.

- Availability of radioisotopes needs to be increased on a global scale (e.g., more generators for diagnostic isotopes, in addition to increased accessibility to theranostic isotopes such as Zirconium-89 and Copper-64).

- The advent of completely automated quality control systems would help save space, decrease operator errors, and expedite the pre-release testing of radiopharmaceuticals.

\section{Streamlining tracer development and clinical translation}

- Strategies to increase the efficiency of discovering new radiotracers for high priority targets are needed.

- Enhanced in silico and in vitro models/methods are required to more accurately predict success of radiotracer candidates and shed light on the molecular basis of non-displaceable binding.

- Animal models that better capture the molecular underpinnings of various diseases are needed for more robust preclinical assessment of candidate radiopharmaceuticals.

- Higher throughput radiotracer development and evaluation in animal models is needed some of which has already begun [51].

- Streamlined methods for optimizing affinity, in vivo stability and pharmacokinetic properties of promising radiotracers is needed. For example, for peptide-based tracers, modifications include but are not limited to cyclization, addition of unnatural amino acids, multimerization, PEGylation and more recently the addition of albumen binders to increase circulation time and improve targeting [52].

- Effective clinical translation and utilization of antibody-based radiotracers that utilize pretargeted approaches and biorthogonal click chemistry.

- Growing costs associated with regulatory compliance are slowing clinical translation and decreasing the number of medical centres that can implement novel radiotracers.

- Successfully validated radiopharmaceuticals for a target of interest (e.g., PSMA) need to be prioritized, as opposed to having 20 probes for the same biomarker.

- Funding mechanisms for IND enabling toxicology studies is needed since these tests are very expensive.

- Regulatory filings need to be expedited without compromising care/quality.

- Reimbursement from healthcare providers is limited for PET tracers, which reduces widespread use/adoption.

\section{Educating the next generation of radiopharmaceutical scientists}

- We are training molecular imaging scientists but what about radiochemists? There is a clear shortage of people with expertise in carbon-11 and fluorine-18 radiotracer development in addition to those with expertise in routine clinical production of radiopharmaceuticals. 
- There are not enough people proficient in kinetic modelling to help determine the best way to quantify imaging data for new radiopharmaceuticals, especially those developed for neuroimaging applications.

\section{Advances in Science and Technology to Meet Challenges}

To address the aforementioned challenges there are a number of emerging technological advances that could make a dramatic impact on the way we approach radiopharmaceutical development and clinical translation. For example, innovations in imaging instrumentation, including time-of-flight PET/MRI and the arrival of total-body PET (TBP) scanners [53] will likely improve our ability to detect a larger range of biomarkers in the broader population. That is, PET/MRI is already enabling more diverse patient populations to be imaged, including paediatric subjects due to reduced radiation exposure (compared to $\mathrm{PET} / \mathrm{CT}$ ). Moreover, $\mathrm{PET} / \mathrm{MR}$ is enabling the precise localization of pain generators in peripheral nerves and/or other parts of the body due to its unparalleled sensitivity, soft tissue contrast, and truly simultaneous collection of molecular and anatomical data (avoiding coregistration issues seen with PET/CT where minor movements between PET and CT scans can introduce significant error when identifying uptake in small nerves) [54]. With respect to TBP, we will likely be able to detect targets that are more diffusively expressed or found at relatively low levels, with only subtle alterations in different disease states, due to the enhanced sensitivity of these systems. Similarly, due to the increased sensitivity of TBP, it will likely also improve accessibility of radioisotopes/radiopharmaceuticals since the required radioactive dose to obtain an adequate signal is significantly lower, meaning that radioisotopes such as carbon-11, fluorine-18 or gallium-68 could be potentially transported over greater distances to cites that do not have a cyclotron or generator.

In terms of streamlining discovery of potential new imaging biomarkers, we need to better leverage data from systems biology (e.g., transcriptomics, metabolomics, proteomics) by implementing artificial intelligence (AI) techniques to extract meaningful information from these large datasets [55]. Al could also be used for tracer discovery and optimization efforts. For example, platforms that use pattern recognition for targets that have a known binder could accelerate probe optimization and improve their success rate [56], especially in terms of predicting BBB permeability.

\section{Concluding Remarks}

PET radiopharmaceuticals will continue to play a critical role in improving the way we understand and treat disease by allowing unprecedented visualization and quantification of molecular processes and pathology in living subjects. To maintain healthy growth and increase innovation in this area of research, strategic training of future radiochemists at the undergraduate and graduate level is vital. Moreover, conversations with relevant funding bodies is needed to create avenues for financial support of expensive toxicity testing and translation of new promising radiotracers. Lastly, we need to bridge the gap between clinical research and clinical practice by having earlier and more frequent communication with regulatory agencies to increase reimbursement of well-validated radiopharmaceuticals that are changing management and improving outcomes for patients.

\section{Acknowledgements}

We would like to thank Dr. Peter Scott, Dr. Anthony Gee, Dr. Matthias Herth, and Dr. Neil Vasdev for their insightful discussions on the grand challenges of the field and possible solutions to enable continued growth and discoveries of new radiopharmaceuticals. 


\section{PET/MRI}

\section{Jinsong Ouyang, Yoann Petibon, Chao Ma, Georges El Fakhri}

Gordon Center for Medical Imaging, Massachusetts General Hospital and Harvard Medical School

\section{Status}

Simultaneous Positron Emission Tomography and Magnetic Resonance Imaging (PET/MR) is an emerging modality with great potential for both clinical and research applications. Since the first installation of clinical simultaneous PET/MR scanner in 2010, the number of PET/MR systems in use has grown rapidly, reaching 190 globally in 2019. Although PET/MR has been largely research based over the last two decades, it is starting to gain traction in clinical care in recent years.

The physical integration of the PET and MR components in a single machine was made possible by a series of engineering achievements that allowed minimizing interference between the two imaging systems [57]. Today's state-of-the-art simultaneous clinical PET/MR scanners include Siemens Biograph mMR, GE SIGNA TOF PET/MR, and United Imaging UPMR790. All systems use lutetium oxyorthosilicate (LSO) or lutetium-yttrium oxyothosilicate (LYSO) crystals coupled with conventional avalanche photodiodes or silicon photomultipliers and $60-\mathrm{cm}$ bore 3-T superconducting magnets. Figure 1 shows a Siemens Biograph mMR whole-body PET/MR, which was installed at Massachusetts General Hospital in 2011, together with PET-MR systems from GE and United Imaging.

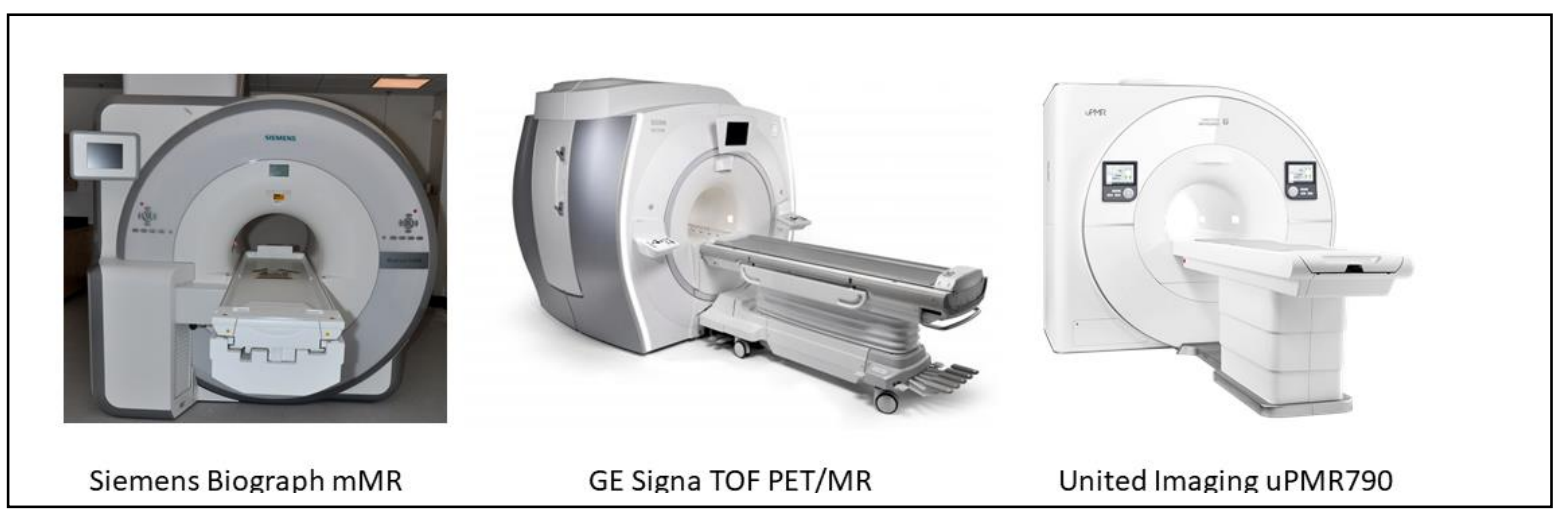

Figure 1. Simultaneous whole-body PET/MR systems from Siemens, GE, and United Imaging (The middle and right pictures were downloaded from GE and United Imaging's web sites).

At its most basic level, PET/MR combines the strengths of PET (e.g. biochemical information, sensitivity) and MR (e.g. excellent visualization of soft tissue, no ionizing radiation), yielding perfectly registered functional, morphologic and molecular information. As a result, clinical applications expected to benefit from PET/MR include oncology, neurology, and cardiology. Furthermore, the information measured with one modality can be used to improve information content of the other modality. For instance, MR-based PET motion correction and PET image reconstruction using MR anatomical prior information can lead to significantly improved PET image quality [58,59]; while simultaneous PET/functional MRI (fMRI) and joint pharmacokinetic modelling can explain the complex spatio-temporal evolution of neurotransmission and cerebral blood volume (CBV) signals that occur during $\mathrm{fMRI}$ studies with a pharmacologic challenge [60]. Finally, combining PET and MR in a single image session enables estimation of physiological processes that otherwise would be impossible with 
PET or MR alone, e.g., quantification of myocardial membrane potential using extracellular volume fraction measured by MR and ${ }^{18} \mathrm{~F}-\mathrm{TPP}+$ volume distribution measured by dynamic PET [61].

\subsection{Current and Future Challenges}

Despite extensive efforts by manufacturers and researchers, the estimation of accurate PET attenuation coefficients remains an unsolved challenge in PET/MR for many organs. The MR signal is a complex function of many variables (e.g., proton density and relaxation times) but not the electron density that determines the photon attenuation property of tissues. The standard MR-based attenuation coefficient estimation method is to segment an MR image volume into different tissue classes (e.g., air, lungs, fat, and other tissues) and then to assign a single attenuation coefficient to each class. However, this method does not account for the intra- and inter-subject variation of the attenuation coefficients, which are significant in lungs and bones and can lead to unacceptable biases in these tissues. Moreover, the smaller transverse field of view of MR compared to that of PET causes truncation of the MR-based attenuation maps in larger subjects, which is another technical challenge that has yet to be fully overcome.

Patient motion during PET/MR imaging is a major challenge for imaging not only in cardiac, hepatic, respiratory, and renal applications but also in brain and virtually any organ. Cardiac imaging is particularly affected by motion artefacts because motion of the heart occurs due to cardiac contraction, respiration, and body movement. For PET, motion contaminates emission data and causes spatial misalignments between the emission and attenuation maps, which in turn results in large quantitative biases in the reconstructed PET activity distributions. These challenges and potential solutions are discussed in detail in section 11. For MR, patient motion leads to artefacts and prolonged imaging time.

Many advanced imaging applications, such as imaging of small brain structures, small tumours, atherosclerotic plaques, or transmural cardiac defects, require a 1-2 mm spatial resolution for both imaging modalities. Although this is currently achievable for $M R$, it is beyond the reach of current commercial PET instrumentation.

In clinical practice, it is often found that a PET/MR scan takes much longer than a PET/CT scan due to the relatively long MR acquisition times. This results in reduced clinical throughput and makes the scan more prone to motion artefacts.

\subsection{Advances in Science and Technology to Meet Challenges}

Recently, it has been demonstrated that MR-based attenuation correction methods employing ultrashort echo time (UTE), zero echo time (ZTE), or combined UTE/multi-echo Dixon acquisitions yield reduced biases in lung and bone regions [62]. Also, it has been shown that atlas-based attenuation correction yielded $<8 \%$ bias in any brain region [63]. When TOF PET data are available, we may be able to jointly estimate emission and attenuation maps (see section 10 ). More recently, deep-learning based methods have been proposed to generate pseudo-CT images directly from MR images for PET attenuation correction, showing encouraging results in generating subject-specific continuous attenuation coefficient maps [64]. 
PET/MR offers a unique opportunity to perform MR-based motion correction of PET data (see section 11). For brain imaging, rigid motion of the head can be measured and tracked using MR navigators or micro-coils [65]. For body imaging, non-rigid motion caused by heart beating and/or respiration can be divided into a number of motion phases. MR images for each phase can be acquired with various techniques to estimate motion fields, which are in turn used to perform motion correction of PET emission and attenuation data. Motion phases can be tracked using ECG, bellows, MR-navigators and/or PET self-navigating signals. Many studies have shown that MR-based PET motion correction results in improved PET image quality. Figure 2 presents results obtained for a dynamic cardiac PET imaging study, with parametric images computed with and without MR-based cardiac and respiratory motion correction [66].

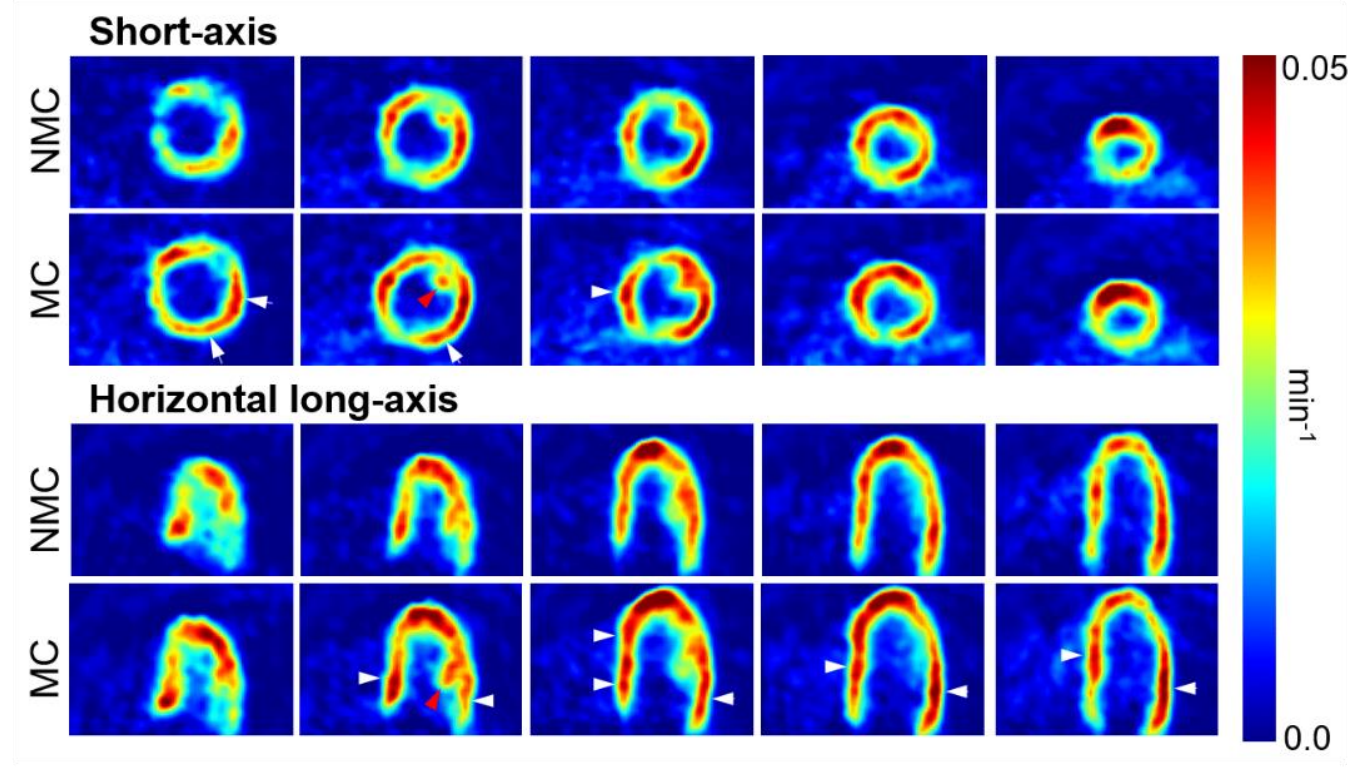

Figure 2. Short-axis and horizontal long-axis ${ }^{18} \mathrm{~F}-\mathrm{FDG}$ consumption rates $\left(\mathrm{K}_{\mathrm{i}}\right)$ slices obtained with $(\mathrm{MC})$ and without (NMC) MR-based PET motion correction for a human ${ }^{18} \mathrm{~F}-\mathrm{FDG}-\mathrm{PET} / \mathrm{MR}$ study. MC yielded higher $\mathrm{K}_{\mathrm{i}}$ values than NMC, especially in regions indicated by the white arrows. Structures such as papillary muscles are also easier to delineate in MC Ki maps (see red arrows).

The spatial resolution of state-of-the-art clinical whole-body PET scanners (e.g., Siemens Biograph Vision digital PET/CT) is $\sim 3.5 \mathrm{~mm}$ (FWHM) near the centre of the field of view. There are various ways to further improve the spatial resolution. The most effective method is to use smaller detector size and depth of interaction encoding although this will likely result in significant cost increase. Also, it is helpful to incorporate accurate point spread function modelling, positron range correction, and high-resolution MR as a prior into the image reconstruction. Finally, if the accuracy of motion fields measured by MR or some external optical tracking device is much better than the PET intrinsic spatial resolution, we may be able to achieve super-resolution for PET by incorporating measured motion fields in the reconstruction [67]. Technically, it is certainly possible for future clinical PET scanners to have a spatial resolution close to that of current small animal PET scanners, i.e., $<2 \mathrm{~mm}$ (FWHM). Such high-resolution scanners will result in more accurate quantitation of small structures in clinical PET images. 
It is essential to minimize MR acquisition time in order to reduce the overall imaging time of a PET/MR examination. MR sequences performed during a PET/MR scan include PET-related ones, i.e., those used for PET attenuation and motion corrections, as well as other clinical ones. Several techniques can be used to accelerate the acquisition of these sequences, including: (a) parallel imaging methods, e.g., sensitivity encoding (SENSE) and generalized auto-calibrating partially parallel acquisitions (GRAPPA), which utilize the distinct spatial distribution of the sensitivity maps offered by phased-array coils for spatial encoding; (b) compressed sensing methods, which leverage the sparsity of an MR image in certain transform domains; and, (c) low-rank based methods, which exploit the spatiotemporal correlations of dynamic MR images. These methods are complimentary to each other and can be combined to further accelerate data acquisition.

Another area of active and promising research lies in radiochemistry with the dual labelling of PET/MR probes that provide complementary information. One good example is the radiolabelling of ferumoxytol (Feraheme), a superparamagnetic iron oxides (SPIO) MR contrast agent, with Zr-89 that allows long term follow up of the tracer and helps map monocyte trafficking in the body, which opens up very promising avenues for monitoring response to immunotherapy with PET and which is impossible with Feraheme alone [68].

\section{Concluding Remarks}

In summary, PET and MR have been successfully integrated with minimal interference between the two systems. Significant progress has been made to solve the issue of MR-based PET attenuation correction. The ability to measure PET and MR data concurrently provide new opportunities for measuring biological processes that would otherwise be impossible to measure with each modality alone. Although it is unlikely that PET/CT will ever be replaced by PET/MR in the future, PET/MRI is gaining traction due to the convenience of a combined exam, reduced radiation dose, and improved PET motion correction. We expect simultaneous PET/MR to offer many opportunities in clinical applications in the future.

\section{Acknowledgements}

We would like to acknowledge grants P41-EB022544, R01-HL118261, R01-CA165221, and R21$\mathrm{MH} 121812$ from the National Institutes of Health. 


\section{Time-of-Flight PET}

Suleman Surti and Joel S. Karp

Department of Radiology, University of Pennsylvania

\section{Status}

Time-of-flight (TOF) PET imaging technique was considered from the very early days of PET and in the 1980s TOF PET systems were developed using $\mathrm{CsF}$ and $\mathrm{BaF}_{2}$ scintillators and photomultiplier tubes (PMTs) with applications primarily in brain and cardiac studies with short-lived isotopes. Despite achieving 400-600ps coincidence timing resolution (CTR), low stopping power and low light output of these scintillators limited the performance of these systems, and led to the adoption of non-TOF systems with other scintillators, primarily BGO due to its higher stopping power and better overall performance for ${ }^{18}$ F-FDG imaging [69]. Early to mid-1990s saw the widespread adoption of ${ }^{18} \mathrm{~F}$-FDG PET as a radiotracer in oncology for cancer detection and staging, ultimately leading to the large growth of PET as a clinical modality, particularly in combination with CT (PET/CT). Development of lutetium-based scintillators in the late 1990s provided a detector material which has stopping power close to that of BGO, but also has a very fast signal and much higher light output, thereby enabling fully-3D PET (without septa) and leading to the development of a new generation of TOF PET scanners from the mid 2000's onwards with system CTR of around 600ps that gradually improved to as low as 450ps with improved detectors and calibration techniques [70]. In recent years there have also been significant advancements in the area of photo-sensor technology and in particular the evolution of silicon-based photomultipliers (SiPMs) that have quickly replaced traditional PMTs in the latest PET systems. Since these detectors are based on semiconductor technology, they can be fabricated in arrays comprised of individual channels that are smaller in size than a traditional PMT and have a much smaller physical footprint. Consequently, the latest PET detector designs have improved light collection with close to, or fully 1-1 coupling schemes where a single small scintillator is read-out by a single SiPM channel. Such a design with high light collection efficiency has improved timing resolution over the light sharing designs that had been used with traditional PMT based detectors. The latest SiPM-based PET systems have system CTR in the range of 210-390ps, as well as excellent spatial resolution $(<4 \mathrm{~mm})$ and high sensitivity due to longer scanner axial length (25 cm or greater) [71-73]. The CTR range of these latest systems represents limitations in detector design due to less than ideal light collection and device noise for those at the higher end, versus those with better light collection and noise suppression (either electronically or via cooling).

The re-introduction of TOF PET in mid-2000s saw significant activity in investigating the advantages of improved CTR for clinical purposes. It had been demonstrated in the 1980s that image signal-to-noise ratio (SNR) was inversely proportional to the square root of the CTR and directly proportional to the square root of the object diameter. New work done after 2000 with more modern reconstruction tools and more realistic activity distributions showed that as the CTR improves, lesion uptake converges faster with iterative reconstruction algorithms leading to significantly reduced noise in the image, in general agreement with the earlier predictions. In practice, TOF PET with improving CTR leads to [74]: (i) better lesion detectability, (ii) shorter scan times, (iii) larger gains in lesion detectability for bigger objects, (iv) more uniform lesion detectability performance over all patient sizes, and (v) reduced variability of lesion uptake measurement over different replicates, different organs, and different patients. Also, TOF provides information that is not necessary for fully tomographic reconstruction (if all data projections are collected) but provides additional consistency requirements in the image 
reconstruction process. Hence, it has been shown that TOF PET images are more robust and less sensitive to errors in collected data leading to applications such as simultaneous estimation of emission and attenuation images from emission data (MLAA) and calculation of attenuation correction factors from emission data (MLACF), both of which will allow generation of quantitative PET images without any CT imaging, and development of PET scanners with more flexible geometry. So, with impressive gains in imaging performance and demonstrated clinical impact from current TOF PET instruments, there continues to be a technical drive towards further reductions (improvements) of the system CTR.

\section{Current and Future Challenges}

Based on recent technological advances, the primary challenge currently is to develop techniques that achieve system wide CTR closer to 100ps with detectors that do not compromise other design characteristics that are important for clinical PET, particularly spatial resolution and sensitivity. The CTR achieved in a PET scintillation detector is affected by:

- the number of scintillation photons detected

- the scintillator signal rise and decay times

- the timing jitter introduced in the detector due to variable depth-of-interaction

- the timing jitter introduced due to multiple reflections of scintillation photons in the crystal

- the timing jitter introduced due to effective SiPM single photon timing resolution (SPTR)

- the uncertainty of the time-pickoff from the electronics signal

Lutetium-based scintillators currently represent the best general combination of properties for use in a PET detector while also providing some of the best CTR results. The best measurements of lutetium based crystals coupled to a single SiPM indicate a CTR of 58-80ps, but this is achieved with small crystals ( $2 \mathrm{~mm}$ thick), whereas a CTR of 98-122ps is achieved with longer crystals ( $2 \mathrm{~cm}$ thick) with higher stopping power appropriate for the needs of a clinical PET system $[9,75]$. These bench-top measurements utilized a SiPM with a very high photon detection efficiency (PDE) and low SPTR values together with high frequency signal readout and/or digital waveform sampling. While these results demonstrate potential for superb CTR, extending these lab measurements to practical PET detectors remains challenging for several reasons - scaling the growth of these new scintillators to high volumes with consistent performance, development of larger SiPM arrays with equivalent PDE and SPTR, and challenges in implementing the complex signal readout and waveform sampling techniques from single detectors into a complete PET system.

Long-term goals of achieving CTR of $<100$ ps will require significant developments in cost-effective techniques to reduce the effects of timing jitter due to the detector, SiPM, and front-end electronics. However, it is clear that 100 ps CTR with lutetium-based scintillators is possible and sets a realistic target for TOF with detectors utilizing the scintillation process in lutetium-based scintillators.

\section{Advances in Science and Technology to Meet Challenges}

Achieving the near future goal of developing a PET system with CTR close to 100ps will require advances in SiPM technology, which combine individual SiPM channels with high PDE into a larger array with a high fill-factor (active sensor area/total sensor area). Early SiPM arrays were fabricated on a common printed circuit board using bond wires to connect individual SiPM devices, leading to low fill-factor and low effective PDE. Recently, SiPM arrays have been produced using Through-Silicon 
Via (TSV) technology to interconnect the individual SiPM channels and significantly improve the fillfactor (> 90\%). Digital SiPMs with integrated time-to-digital converters on the chip provide the capability to perform fast timing by triggering off the first detected photons, thereby eliminating the need for high frequency analogue signal readout and/or waveform sampling. Currently, the Philips digital photon counter (PDPC) provides this capability and has shown direct scalability from bench-top measurements to a full system $[73,76]$. Due to the original fabrication technique, the fill-factor of these devices is low, leading to a lower PDE ( 25\%) - a technical limitation that will be overcome in the near future.

To achieve < 100ps CTR with lutetium-based scintillators will, however, require development of practical methods that measure depth-of-interaction (DOI) of the gammas within the scintillator to minimize the uncertainties of the time pick-off and also reduce the effect of multiple photon reflections on the timing jitter. While methods to measure DOI in a pixelated detector exist, removing the impact of multiple reflections of scintillation photons within a small narrow crystal is challenging. Alternatively, position-sensitive monolithic detectors with much wider cross-section (e.g., $4-25 \mathrm{~cm}^{2}$ ) intrinsically have fewer reflections from the walls and have been shown to provide spatial and timing resolution that is as good or better than pixelated detectors while also enabling the measurement of DOI. Since scintillation light from events will now be spread over multiple SiPM channels some form of timing skew correction is required. In fact, it has been shown that a spatial resolution of $<1.5 \mathrm{~mm}$ (FWHM) and CTR of $<150$ ps can be achieved in a $2 \mathrm{~cm}$ thick LYSO crystal coupled to a PDPC array [77]. It is conceivable that a digital SiPM array with higher PDE will lead to further gains in the CTR achieved in a monolithic detector. Another possible approach will be to build a multi-layered detector with thin slices of scintillator, each with independent read-out. In principle this type of detector would enable DOI and have excellent timing performance due to elimination of depth effects. However, a practical implementation with side read-out becomes challenging. With all these developments it will still be challenging to achieve system CTR values better than the $58-80 \mathrm{ps}$ currently measured on the benchtop with small crystals on a highly optimized readout scheme $[9,75]$.

Any further reduction in CTR values will therefore require development of either a new high light output scintillator with a much faster scintillation signal rise time or measuring event timing using a property other than the scintillation mechanism. One avenue could be the signal from very fast Cerenkov photons (few ps time scale that is an order of magnitude or faster than the scintillation process in lutetium-based scintillators) that are produced due to the passage of charged electrons produced by the annihilation photons within a scintillator. The number of Cerenkov photons produced is very low and so much higher SiPM PDE values may be needed to achieve high signal-to-noise properties. In addition, the SPTR of current SiPM devices will be a limiting factor in maintaining the fast timing characteristics of the Cerenkov signal. As shown in Figure 1, the average Cerenkov photon production of 10-20 in LSO and best measured SPTR in current SiPM devices (100-200ps FWHM) will not lead to a large gain in CTR over the scintillation mechanism. However, SPTR of $\sim 20 p$ s has the potential to reach a CTR of 30ps in a small LSO crystal. With careful electronics design and signal processing the SPTR of some existing devices can be reduced, but achieving SPTR value of $\sim 20$ ps will require a significant re-design of the SiPM devices [78]. Long term, there are efforts also underway to meet the challenge of achieving 10ps TOF resolution that are likely to introduce new quantitative imaging capabilities [79]. 


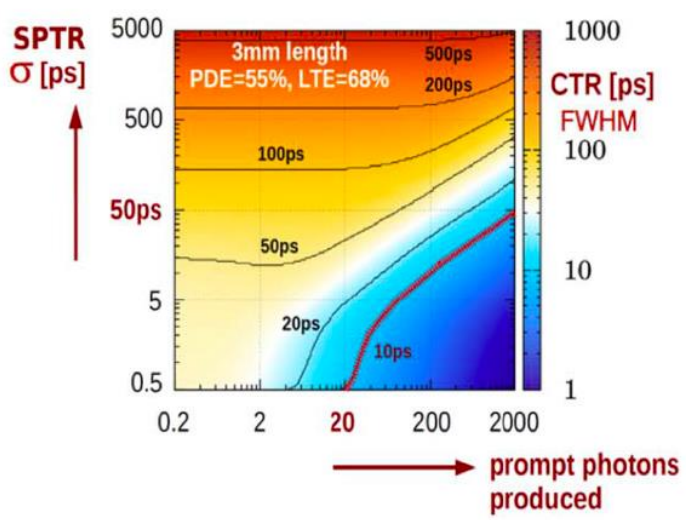

Figure 1. Cramér-Rao Lower Bound [80] calculations for CTR in a $2 \times 2 \times 3 \mathrm{~mm} 3 \mathrm{LSO}: \mathrm{Ce}, \mathrm{Ca}(0.4 \%)$ crystal for varying SiPM SPTR and the number of 'prompt' Cerenkov photons produced in the crystal [81].

\section{Concluding Remarks}

In a relatively short time period TOF PET has established itself as the gold standard and fast timing detectors have become an integral component of today's state-of-the-art PET/CT (and PET/MR) systems. Although the TOF PET/CT systems introduced 15 years ago had far superior imaging performance compared to the first TOF systems from the early 1980's they did not have improved timing resolution. Due to availability of fast solid-state photo-sensors (SiPMs) and motivated by the demonstrated clinical benefit of TOF PET, we now have systems with close to 200 ps TOF resolution. It is remarkable that the rate of improvement is not slowing down and an improvement to $100 \mathrm{ps}$ TOF resolution seems imminent. Perhaps even more significant is that the technology required to achieve this level of timing performance will not require trade-offs in other crucial factors contributing to image quality, notably high sensitivity and spatial resolution. The combination of fast scintillators with high light output and high stopping power together with fast solid-state photo-sensors with high photo-detection efficiency and low noise has made this possible. Future improvements in fast timing detectors and TOF resolution will not only lead to higher signal-to-noise and better image quality, but also improved quantitative accuracy of PET.

\section{Acknowledgements}

We would like to acknowledge funding support from NIH grant numbers R01-CA113941 and R01CA196528. 


\title{
8. Total-Body PET
}

\author{
Ramsey D. Badawi ${ }^{1,2}$, Simon R. Cherry ${ }^{2,1}$ \\ 1 Department of Radiology, University of California, Davis \\ 2 Department of Biomedical Engineering, University of California, Davis
}

\section{Status}

Positron emission tomography (PET) images, whether produced for medical research or clinical diagnostic purposes, have always been limited by counting statistics, leading to relatively high noise levels in the reconstructed images. Image noise is frequently mitigated by spatial smoothing, resulting in increased partial volume effects which degrade quantitative accuracy. For parametric images derived from kinetic models, noise in the data can result in bias as well as imprecision. Thus, a major focus for PET instrumentation and methodology research has always been on approaches to improve the detection efficiency (usually known as the sensitivity) of PET scanners to increase the number of recorded events per unit activity. PET scanner designs have evolved in a logical fashion, and in concert with advances in technology and image reconstruction, from the earliest single-slice scanners, to multi-slice scanners, and finally to multi-slice scanners that utilize the coincidence data acquired not just within each slice ("two-dimensional acquisition") but also between each of the slices ("threedimensional acquisition"). Nonetheless, the detection efficiency for a single organ is still only on the order of 2-3\%, and for protocols that involve translating the subject to cover most or all of the body, the overall detection efficiency is less than $1 \%$.

Total-body (TB) PET seeks to make the next step change in detection efficiency, by extending the detectors from coverage of $15-30 \mathrm{~cm}$ axially along the body, to coverage of the entire human body ( 2 meters) (Figure 1). By dramatically improving the geometric coverage and collecting more of the isotropically-emitted radiation, simulations predicted [82] that the detection efficiency for imaging single organs could be improved by a factor of up to 4-5, and for applications involving imaging the entire human body (e.g. in staging and response to therapy for melanoma), the increase in detection efficiency could be a factor of 25-40, compared with conventional PET scanners. Such large increases in sensitivity may be used to reduce noise levels in the images (improving precision, and by facilitating improvements in spatial resolution, also accuracy), or to acquire data much more quickly or at lower injected doses. In addition to allowing current PET protocols to be performed better, faster or with less dose, TB PET opens up new research opportunities through its ability, for the first time ever, to image radiotracer kinetics in every single organ and tissue of the human body simultaneously [35].

The first TB PET/CT scanners, as well as other scanners with large axial fields of view (70 $\mathrm{cm}$ or more), have recently been developed, commercialized, and are beginning to be applied both in research and clinical settings $[1,2]$. The challenges and opportunities provided by total-body and large axial field of view scanners has led to a rapidly growing scientific effort across all areas of instrumentation and methodology related to PET. 

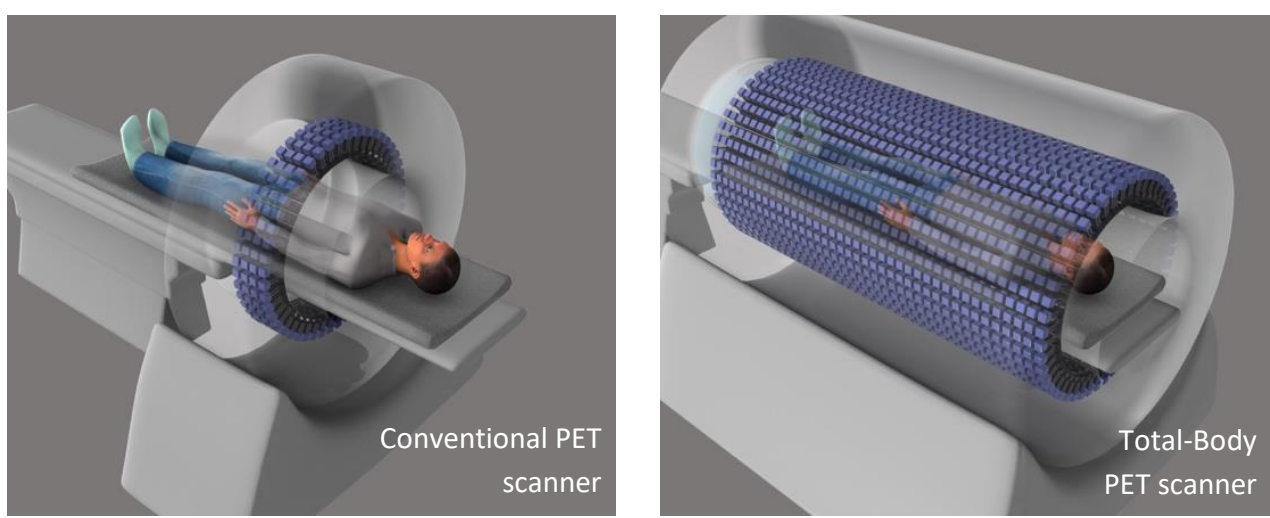

Figure 1. Concept of total-body PET with complete coverage of the human body approaching maximum geometric collection efficiency in comparison to conventional PET scanners which have an axial coverage of between 20 and $30 \mathrm{~cm}$. (Reproduced with permission from [40]).

\section{Current and Future Challenges}

Whilst the performance of the first TB PET/CT scanner has already produced data consistent with the computer simulations, considerable challenges remain with regards to the practical application of quantitative TB PET across the gamut of potential applications. Examples include:

\section{Motion}

Motion impacts virtually all scanning tasks in PET and is exacerbated in TB PET as lines of response passing through regions with motion can affect (primarily through attenuation mismatch with the CT) other regions some distance away due to the large axial acceptance angle. Since the whole body is in the field of view for the entire scan duration, all motion that occurs during the scan is captured. Motion can cause artefacts in the images and increase the partial volume effect in non-isotropic ways - degrading lesion detectability and contrast, impacting quantitative accuracy and causing errors when generating parametric images. Accurate total-body parametric imaging, in particular, will require a comprehensive approach to motion correction. Motion falls into several categories:

- Gross motion of subject's limbs, torso and head

- Regular or semi-regular physiological motions due to the cardiac cycle and respiration

- Gastrointestinal (GI) motions, e.g. stomach emptying and peristalsis

- Genitourinary (GU) motion - bladder filling

Correction methods for some of these motion types are quite mature (e.g., brain motion), but most have yet to be fully addressed (see section 11). For example, while both cardiac and respiratory motion have been carefully studied, the problem of combined cardiac and respiratory motion in the context of dynamic imaging has barely been explored. Similarly, there is little work on correction for GI motion or bladder filling.

\section{Data corrections}

Another challenge is in quantitative imaging at low radiation doses. While TB PET offers the potential for a reduction in injected activity of 20-fold or more, this is of limited value if a high-dose CT scan must be performed for attenuation correction. While simultaneous reconstruction of emission and attenuation maps is possible as discussed in Section 10, this becomes more challenging with the sparse data expected with very low-dose scans. 
TB PET also offers the possibility of acquiring data across multiple organs at very short frame durations (1 second or less) (Figure 2) [83]. This presents a huge opportunity for investigation of fast kinetics, physiological motion and other questions yet to be developed. However, this rapidly changing and data-sparse environment presents challenges in terms of (a) reconstructing accurate images and (b) generating accurate scatter correction estimates. A further challenge is the increased computational burden that arises for such highly temporally sampled image sets.

Other areas that remain to be explored include validation of randoms corrections for studies using short-lived radionuclides (where singles rates, dead-time and multiple coincidences may be very high and rapidly changing), and, increasingly of importance, accurate randoms and scatter corrections for "dirty", yet useful, radionuclides that have a significant fraction of emissions that are not positrons.

\section{Total-Body Parametric Imaging}

For accurate parametric imaging with TB PET, it is necessary to determine radiotracer delivery (the "input function") to all tissues of interest. Clearly, the time delay between radiotracer bolus injection and delivery, and the dispersion of the bolus, are dependent on the position within the body. Delay and dispersion must be estimated if kinetic models are to give unbiased results. Some organs - e.g., the lung and the liver, have both venous and arterial supply, and need more complex models. Other organs may also require specific models - for example, the standard three-compartment model for fluorodeoxyglucose is not applicable to the kidney. Finally, while TB PET offers the promise of always being able to obtain an image-derived input function, since there is always a major blood vessel in the field of view, this ability may be confounded by the presence of metabolites in the blood.

\section{Advances in Science and Technology to Meet Challenges Motion}

Gross motion outside of the brain is a non-trivial problem, however we can build on existing work in deformable image registration [84]. Data-driven respiratory (and cardiac) gating research is also mature, with a number of vendors offering "motion-frozen" image generation options even for conventional scanners. However, these methods typically involve averaging of data across gates, which is not directly helpful for capturing radiotracer kinetics rather than a single static image. Both of these motion types could be addressed by the development of a new class of spatio-temporal reconstruction algorithms [85] that aim to determine motion fields and incorporate these directly into the reconstruction process, so that a motion-corrected image series (or a static parametric or activity concentration image) may be generated directly. This approach could conceivably also help with motion due to bladder filling. These and other potential solutions are discussed further in sections 10 and 11.

GI motion is a tougher proposition. While images may be impacted by GI motion, the Gl tract may not be readily apparent in the data. It may be necessary to use data from radiotracers that do show increased GI uptake (e.g., fluciclovine) to develop and validate the methods.

\section{Data corrections}

A common issue for a number of the data correction problems described above is noise. There are a variety of promising de-noising techniques currently being investigated, including the use of deep learning approaches, either separate from, or incorporated into, the reconstruction algorithm [86]. 
For attenuation correction, the background radiation from Lu-176 present in lutetium-based detectors could be used as an additional data source [87]. Both motion correction, and low-dose attenuation correction could be facilitated by development of total-body PET/MR systems, however there are significant technological challenges and cost implications for an MR system to achieve a homogeneous field over such a large volume if the intent is to match the imaging field of view of a TB PET system. Computational challenges must also be addressed in terms of speed, data transfer bandwidth and data storage. Computational problems actually cut across all aspects of TB PET practice and will need to be addressed if TB PET is to fulfil its promise.

\section{Total-Body Parametric Imaging}

Comprehensive models that account for delay and dispersion of the radiotracer delivery, as well as the different physiology of different organs need to be developed and validated to make accurate TB PET parametric imaging a reality. Metabolites remain a problem to be addressed, although in many cases this can be accomplished with a small number of venous blood samples rather than arterial sampling. The possibility of using data derived from the liver (which is always in the field of view) to model metabolite generation directly from the data has also been suggested. Early results from very rapid temporal sampling during tracer delivery suggest the presence of additional physiologic information, however this requires detailed investigation and explanation. Clearly, much more work is required.

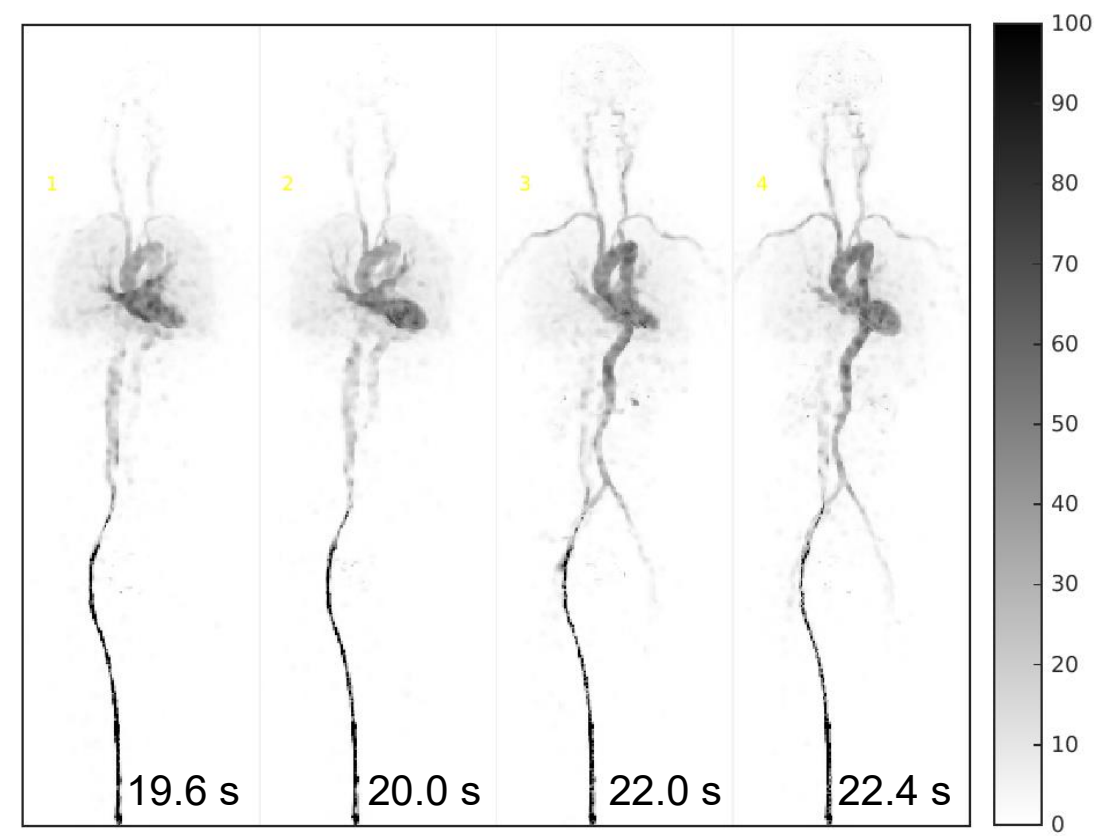

Figure 2. Dynamic total-body PET images, each collected over just 100 milliseconds, showing the distribution of ${ }^{18} \mathrm{~F}-$ fluorodeoxyglucose through the vasculature shortly after injection. Changes between systole and diastole are apparent. Times indicated on the bottom are the time after initiation of bolus injection.

\section{Concluding Remarks}

Total-body PET is now a reality, with first systems installed, and a number of companies and academic labs designing very large axial field-of-view PET scanners. Initial results are tremendously encouraging. The unprecedented sensitivity of these systems opens new opportunities for the use of PET clinically, as well as in biomedical research, and present both challenges, and new frontiers, for PET physics and methodology experts to explore. 


\section{Acknowledgements}

The authors thank all members of the EXPLORER Consortium past and present, as well as our many colleagues who have made valuable contributions and influenced our thinking. This work was supported in part by NIH grants R01 CA 170874, R01 CA206187 and R01 CA249422.

\section{Disclosure}

UC Davis has a research agreement and a revenue sharing agreement with United Imaging Healthcare. 


\section{Application-specific PET systems}

\section{Taiga Yamaya and Go Akamatsu}

National Institute of Radiological Sciences (NIRS), National Institutes for Quantum and Radiological Science and Technology (QST), Chiba, Japan

\section{Status}

High spatial resolution can contribute to minimization of the partial volume effect in PET, especially when imaging small structures. High sensitivity is also required to obtain low-noise images, especially for dynamic imaging with short time frames. To maximize the quantitative accuracy of PET, both characteristics of spatial resolution and sensitivity should be further improved even though they are basically in a trade-off relationship. In addition, production cost should be addressed to make PET diagnoses more available.

Organ-dedicated PET systems have high potential to realize high spatial resolution and high sensitivity without increasing manufacturing cost compared with general-purpose whole-body PET systems. Placing the detectors closer to the subject can increase sensitivity because of the enlarged solid-angle coverage for the field-of-view. Better spatial resolution will also result due to the reduced photon noncollinearity effect. A good example can be seen in small animal PET, which provides accurate preclinical quantitative images with high resolution detectors [88]. The research and development for organ-dedicated PET systems has been motivated by unmet clinical needs for imaging small tissues and lesions. Many PET systems dedicated for specific organs, such as the breast, brain, heart and prostate, have been developed $[41,89]$. Following the commercialization of some cylindrical systems such as HRRT and MAMMI [89], novel systems with non-cylindrical detector arrangements have been prototyped (Figure $1(\mathrm{a})$ ).

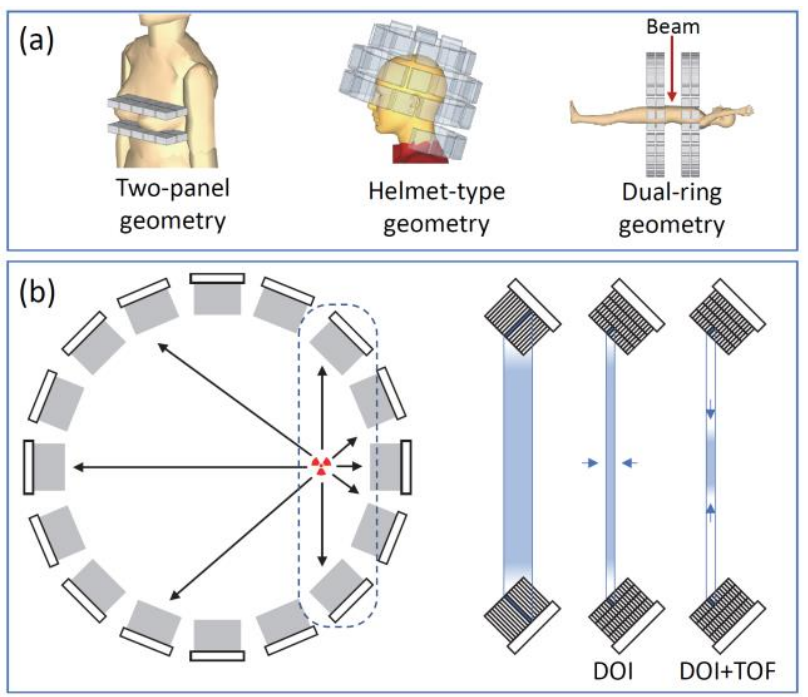

Figure 1. Representative application-specific systems and their detector arrangements (a), and schematic illustrations of effect of depth-of-interaction (DOI) measurement and time-of-flight (TOF) measurement on localization accuracy (b).

Another role of application-specific PET systems is in image guidance for therapy. A good example is in-beam PET, which is a PET application for in-situ range verification in particle therapy such as using a proton or carbon ion beam. While technologies for ion therapy with proton and carbon ion beams have been remarkably advanced in the last few decades, an in-situ monitoring method is still not wellestablished. Uncertainty in the range can be confirmed by imaging the distribution of positron 
emitters produced through fragmentation reactions along the beam path. Various system designs such as dual-head, partial-ring, dual-ring and shifted-ring geometries, have been studied [90]. Using PET to monitor tumours in motion (e.g. due to respiration) during therapy is also of interest. For example, a combined Linac-PET system has been developed for PET-based biology-guided irradiation [91]. Other examples that were developed for image guidance in general surgery are a PETlaparoscope system [92] and a PET-endoscopy system [93].

\section{Current and Future Challenges}

One of the big challenges in developing application-specific PET systems is in radiation detectors. In order to maintain sufficient detection efficiency for $511 \mathrm{keV}$ photons, scintillators should be not only dense enough but also long enough, $2-3 \mathrm{~cm}$ long. The thickness of the scintillation crystals causes parallax error; spatial resolution is degraded when radiations are incoming obliquely. The effect of parallax error is enhanced in compact PET systems with small ring diameter, which is often seen in application-specific PET systems. Depth-of-interaction (DOI) measurement will be a key technique to minimize the parallax error while maintaining sufficient efficiency. A lot of DOI coding methods have been investigated, and [94] reviews them. Among them, a two-layer DOI detector with pulse-shape discrimination has been used in a commercial brain PET system, and a four-layer DOI with reflector control has been used in a commercial breast-dedicated PET system.

Another key technology for application-specific PET systems is time-of-flight (TOF) measurement [95]. TOF is usually employed to improve image signal-to-noise ratio. TOF is also used to reduce image artefacts in application-specific systems with a limited angle tomograph geometry, such as a dualhead geometry and a partial-ring geometry. The benefits of TOF information are effective to overcome the limitations of such non-full-ring designs.

It should be noted that the big future challenge is to make it possible to visualize in-vivo small structures quickly and quantitatively. This challenge is not limited to application-specific systems. Continuing efforts to explore better spatial resolution and higher sensitivity are needed to address this big challenge.

\section{Advances in Science and Technology to Meet Challenges}

As mentioned above, DOI and TOF would be key technologies to improve the performance of dedicated PET systems. However, PET detectors with a combination of DOI and TOF capabilities have not been studied well. A novel detector design that can achieve both DOI and TOF capabilities is expected as a practical detector for next-generation PET systems (Figure 1 (b)).

An innovative imaging scheme beyond that of current PET systems is warranted to meet the big challenge mentioned above. For current typical PET systems, the sensitivity of coincidence detection does not exceed $10 \%$. This means over $90 \%$ of the decays do not contribute to imaging. In other words, there is plenty of room for sensitivity improvement in current PET systems. Application-specific systems (including small-animal systems) offer a practical method to improve the sensitivity with a limited number of detectors.

Further sensitivity gain can be expected by applying a Compton camera technology for PET. In a Compton camera, the activity source position can be localized on the surface of a cone by measuring 
an event which causes Compton scattering in a scatterer detector and photoelectric absorption in an absorber detector. As the singles count rate is usually 10 times higher than the coincidence count rate in typical PET scans, there are a lot of single gamma events which do not make coincidence pairs, and these events can be used for imaging by the Compton imaging method. Compton cameras have been developed by many groups, and one of them was clinically applied recently [96]. However, current Compton camera technologies suffer from limited quality of images, which is generally much lower than that of PET images due to limited energy resolution, sensitivity and projection angles.

One realization of the combined Compton-PET system has been a multi-cylinder detector geometry, where the inner detector ring works as a scatterer and the outer detector ring works as an absorber in Compton imaging [97]. PET measurement is also possible by taking the outer-outer coincidence as well as the inner-inner coincidence and the inner-outer coincidence. This concept is known as whole gamma imaging (WGI), and the first prototype successfully showed a $909 \mathrm{keV}$ Compton image of a ${ }^{89} \mathrm{Zr}$-injected mouse, which was almost equivalent to the PET image obtained from the same ${ }^{89} \mathrm{Zr}$ distribution (Figure 2). Although data correction methods for Compton imaging (e.g. attenuation correction and scatter correction) are yet to be developed for quantitative imaging, combined Compton and PET imaging is expected to extend systems for clinical use once this has been achieved.
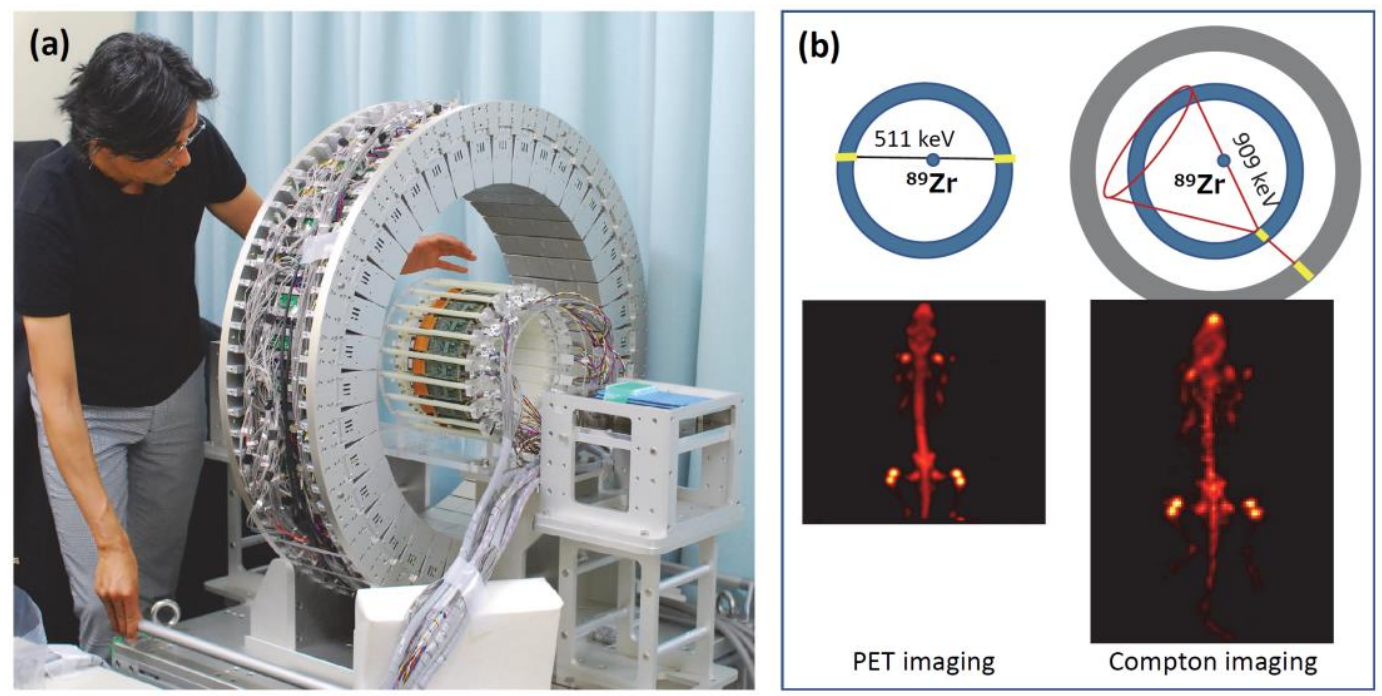

Figure 2. A whole gamma imaging (WGI) prototype, which is a PET system combined with a Compton camera (a). In a mouse imaging demonstration (1-hour measurement started 22 hours after $9.8 \mathrm{MBq}{ }^{89} \mathrm{Zr}$ injection), the Compton image of $909 \mathrm{keV}$ gamma rays was comparative to that of a PET image (b).

Another potential use of WGI is triple-gamma imaging or $\beta^{+}-\gamma$ coincidence imaging. Scandium- $44\left({ }^{44} \mathrm{Sc}\right)$, which emits a positron (i.e., a pair of $511 \mathrm{keV}$ photons) and a $1157 \mathrm{keV}$-ray almost at the same time, is a good example of such a source. The source position can in principle be localized at the intersection points between a line-of-response and the surface of the Compton cone. This novel direct imaging method may realize in-situ real-time tracking of tiny activity source such as a single cell in a regenerative treatment, although further improvement is required in detector performance parameters such as energy resolution, timing resolution and efficiency to realize such a novel concept.

Given that such application-specific PET systems are in wide use already, there are new potential opportunities for clinical research and practice. In the field of particle therapy, a new quantitative biomarker might be found by using PET-guided monitoring. For example, the washout rate of 
radioactivity produced through fragmentation reactions may reflect the biological condition of tumours [98]. New potential PET applications such as PET-guided surgery and biopsy as well as dosimetry for targeted radioisotope therapy and boron neutron capture therapy are also drawing the attention of researchers.

\section{Concluding Remarks}

Recent remarkable advances in PET detectors have facilitated realization of flexible system designs. PET systems optimized for specific organs or applications have the potential to meet presently unmet clinical needs and to create new opportunities for biomedical research. In addition to continuous work to develop novel PET systems, researchers need to explore alternative imaging concepts beyond those of current PET systems.

\section{Acknowledgements}

The authors were supported in part by the QST President Fund, the QST-IRI Program, the JSPS KAKENHI grants (18K19949, 19KK0280 and 20H00671) and the AMED program (JP2Ohe2202004). 


\section{Image reconstruction and joint estimation \\ Georg Schramm, Ahmadreza Rezaei, Johan Nuyts \\ Department of Imaging and Pathology, Nuclear Medicine \& Molecular imaging, KU Leuven}

\section{Status}

In recent years, the size of the PET detectors has been decreasing, the time-of-flight (TOF) resolution has been rapidly improving and the axial extent of the PET systems has been increasing continuously. As a result, the total number of TOF bins and lines of response (LOR) is increasing spectacularly, making the size of conventional sinograms inconveniently large. In addition, sinogram storage is becoming less efficient, because in many clinical procedures, most PET sinogram TOF bins will not acquire any count at all, and list mode reconstruction is more and more used as an alternative.

The improving TOF resolution offered by current PET systems increases their effective sensitivity (i.e. the information provided by each event). It also enables to estimate not only the activity distribution in the patient body from the patient PET-scan, but also the attenuation and/or the detector pair sensitivities. These estimates can, in turn, be used to refine or even replace the information obtained from transmission measurements and/or normalization procedures. It has been found that with TOF, conventional PET imaging is more robust against system matrix errors such as errors in the attenuation map [99].

Since PET data are noisy and tomographic reconstruction is an ill-posed problem, regularization of PET image reconstruction is necessary. This is typically done by stopping the iterations early, by smoothing the reconstructed image, or by using maximum a posteriori algorithms, i.e. by combining the likelihood (data fidelity term) with a function (known as a prior or a penalty) that encourages desired features, such as smoothness or similarity to other available images of the same patient. Regularization with priors has been an active research area for many years, and recently it has finally found its way into commercial reconstruction software. Noise suppression with deep learning methods has been successful in many fields and is currently being investigated by many colleagues for regularization during or after image reconstruction.

\section{Current and Future Challenges}

With current TOF resolution (approx. 200 ps) and axial FOVs of approximately $25 \mathrm{~cm}$, storing a complete TOF sinogram uses tens of Gb of memory. This size, but also the sparsity of binned raw data, will substantially increase with longer axial FOV, improved TOF resolution, smaller detector crystals, depth of interaction detection and the potential use of the photon energy. The two main approaches to remedy this are working with list mode data and reducing the sinogram size by decreasing the sampling accuracy.

In list mode acquisition, the emission data are stored as a chronological list of detected events. It is not only becoming more efficient than the conventional sinogram data representation, it also facilitates storage of additional information, in particular the energy of both photons. In combination with refined scatter estimation models, these energies could be used to reduce the influence of scatter from outside the field of view, and to improve the signal to noise ratio by giving more weight to photon pairs that are more likely to be true coincidences. Many list mode reconstruction algorithms have been developed and validated, but there are still some novel ones to be developed, such as a 
convergent list mode algorithm using non-differentiable priors, methods for efficient position dependent resolution modelling, and adaptations for list mode to methods for joint estimation of the activity and attenuation from TOF-PET data. List mode reconstruction requires access to the sensitivity and scatter contribution for each LOR, which are typically still stored as non-sparse sinograms. Fortunately, the sensitivity sinogram is non-TOF, and the scatter contribution can be well represented at low resolution, because it is (in most cases) spatially smooth.

As the TOF resolution increases, the angular sampling requirements become less demanding, enabling data size reduction by using very aggressive sinogram rebinning techniques [100]. However, these methods create new challenges. Sinogram rebinning involves combining TOF-events that have seen different attenuation and detector sensitivities, which can be solved by binning the conventional nonTOF attenuation and sensitivity sinograms into a TOF sinogram of effective LOR and TOF-bin dependent sensitivities. It is not clear if joint estimation techniques can be successfully applied to such rebinned sinograms. The problem can also be avoided by precorrecting the sinogram before rebinning; this deviation from the Poisson model is expected to become less harmful as TOF-resolution improves.

Analytical techniques such as reconstruction with filtered backprojection or the use of consistency conditions for joint estimation or calibration purposes usually require access to high resolution TOF sinograms. If it is found that (some of) these methods cannot be applied to rebinned sinograms and/or list mode files, then alternative lossless sinogram compression techniques may be required, such that parts of the full sinogram can be expanded on the fly as needed.

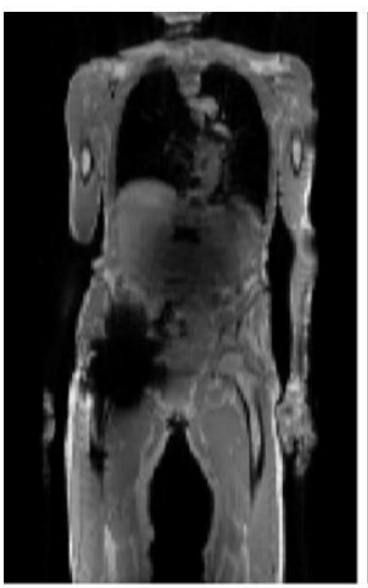

(a)

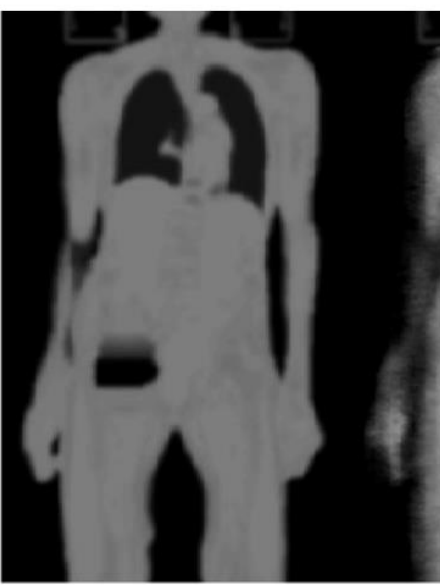

(b)

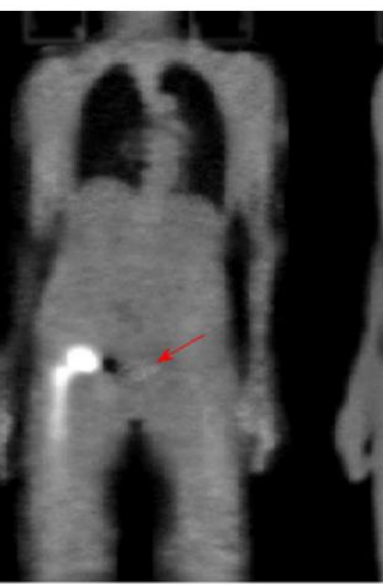

(c)

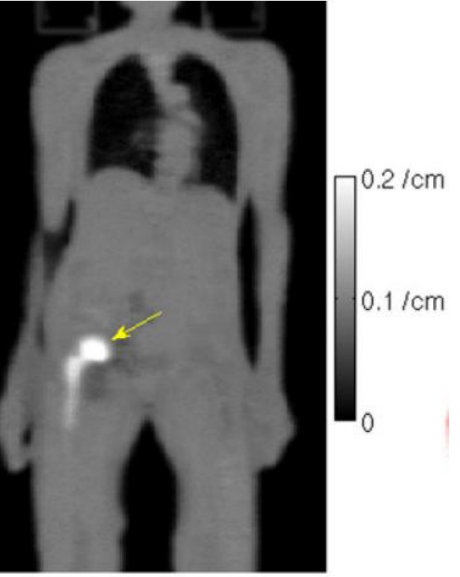

(d)

Figure 1. Coronal slices of In-phase Dixon MR (a), MR-based attenuation image using standard tissue segmentation (b), and attenuation images from TOF joint estimation without and with MR-based prior (c) and (d), respectively. Note that a metal hip implant caused a huge signal void in (a) that translated into (b). The shape and higher attenuation of that implant are nicely recovered in (c) and (d) leading to more correct local attenuation. Reprinted (part of Fig. 2) from [101].

Further important challenges for PET image reconstruction are more accurate modelling of photon attenuation and scattering, improving the resolution of the reconstructions at a given noise level (bias vs noise trade-off), and correction for patient motion. Correction for photon attenuation using external information from CT or MRI poses in many cases no problems. However, there are cases where the information provided by CT or MRI is incorrect or where no such information is available at all. These include regions affected by (respiratory and cardiac) motion in PET/CT and PET/MR, regions affected by metal implants in PET/CT and PET/MR, all regions where reliable MR-based attenuation 
correction is challenging (e.g. the lung, or flexible MR coils), and organ-specific "stand-alone" PET systems (e.g. helmet brain PETs, see section 9). Respiratory and cardiac motion often create mismatches between the CT-derived attenuation and the activity distribution, because CT typically captures only a single phase of the cyclic motion, while the MR image may suffer from motion artefacts. In PET/MR, many MR sequences are usually applied, and as a result, most of the PET data are not acquired simultaneously with the MR image for attenuation correction. In PET/CT, the attenuation and activity images are never acquired simultaneously. As a result, any patient motion after the CT or MR acquisition corrupts the attenuation image. In all those cases, joint estimation of activity and attenuation (MLAA) from the PET raw data themselves, can help to improve the accuracy of the reconstructed PET images as shown in Figure 1. However, it is known that the time-of-flight PET data determine the attenuation sinogram only up to a constant [102]. A reliable, robust and general method to estimate that constant in different applications (e.g. via the introduction of prior knowledge) is one of the unsolved questions and a subject of active research. With improvements in the TOF resolution of future scanners, accurate TOF-based calibrations become increasingly important. It has been shown that compared to conventional MLEM reconstructions, joint estimation techniques are more sensitive to timing calibration errors (timing offsets and time resolution [103]).

A quantitatively accurate reconstruction of the tracer distribution requires correction for the additive randoms and scatter contributions of the measurements. Estimation of the randoms component (which is independent of TOF) will possibly remain unchanged, and will be (as it currently is) corrected for by either directly measuring a crystal map of the singles rates or by indirectly estimating them from measurements of a delayed window. An accurate estimation of the amount and distribution of scattered coincidences still remains a challenge in most available PET scanners. Extra attention has been paid over the years to accurately simulate and correct for double-scattered in addition to singlescattered events [104]. Nevertheless, the "tail-fitting" process to account for multiple scatter and outof-FOV scatter (and at times multiple scatter) remains a source of uncertainty which affects quantification of both standard reconstruction techniques and joint reconstruction methods. With improving TOF resolution, special attention also needs to be paid to the TOF distribution of the simulated scatter. An alternative to simulating the scatter could be using separate energy windows for scatter and prompts, assuming that better energy resolution of future detectors will enable this.

As the achievable reconstructed resolution continues to improve due to advances in PET detector hardware and system design, correction for even small amounts of motion, that did not have a clinically relevant impact on the image quality in current systems due to their limited resolution, will become more and more important. This is especially true for long dynamic brain acquisitions and regions affected by respiratory and cardiac motion. During the last decade, a lot of progress has been made in the field of motion correction. Unfortunately, application of motion correction techniques in clinical routine is still very limited, which might be due to a possible overhead of required external hardware and patient preparation. Fortunately, it has been shown that at least for some tracers (in particular $\left.{ }^{18} \mathrm{~F}-\mathrm{FDG}\right)$, data-driven motion correction techniques work well, especially in the compensation of rigid brain motion [105] and non-rigid periodic motions [106]. An important open problem is the development of a robust purely data driven approach to reconstruct a single PET image from all acquired raw data, which estimates and compensates for non-rigid respiratory and cardiac motion. More details on motion correction are given in section 11 . 
Another persistent challenge is, first, how to improve the prior information that is used in maximum a posteriori PET reconstructions, and second, how to efficiently tune the hyper-parameter, i.e. the weight that balances the influence of the data fidelity term and the prior. As the number of proposed priors and reconstruction methods strongly increases, the ways those methods are tuned and evaluated for a given clinical task have to be improved. This usually involves observer studies by clinical experts. Since their availability is limited, the efficiency of these studies should be maximized. It remains to be seen if mathematical observers or learned observers can help in this challenge.

\section{Advances in Science and Technology to Meet Challenges}

As argued in section 7, it is likely that TOF resolution of around 100ps will be achieved in the next generation of PET scanners. Improved TOF resolution will be beneficial for PET image reconstruction in several ways. First of all, it will increase the signal-to-noise ratio of the images and the convergence speed of MLEM. Second, back-projections of (pre-corrected) data will come closer and closer to the desired final reconstruction such that real-time PET reconstruction might be possible. Third, better TOF resolution will lead to an even better performance of joint estimation of activity and attenuation (see e.g. the variance analysis in [102]). And finally, as the TOF resolution improves, the angular sampling requirements become less restrictive, and they will completely vanish when the TOF resolution goes below about $30 \mathrm{ps}$, enabling high quality PET imaging using unconventional detector configurations. As mentioned above, full exploitation of the TOF information requires very accurate timing calibration, but as the PET data become richer, "self-calibration" based on the patient PET data themselves [103] will become more accurate and robust too.

Advances in detector technology will lead to more precise spatial and temporal detection of the photons and the use of new crystal materials such as $\mathrm{LaBr}$ could improve the energy resolution. These developments will not only increase the achievable reconstructed resolution, they may also provide new information about the trajectory history of the photons in the detector, that can be used during reconstruction [107]. Together with the ever increasing axial FOV (with as an extreme example the total body PET systems $[1,108])$, this will create new challenges (e.g. data size) but also many opportunities (e.g. whole-body dynamic reconstruction, correction for out-of-FOV scatter).

As mentioned above, PET images must be reconstructed at finer spatial resolution from PET scans that grow quickly in size, creating a computational challenge. Fortunately, when the TOF resolution improves, the iterative algorithms converge in fewer iterations and the angular sampling can be reduced without adverse effects on the final image quality. In addition, the computing power continues to increase as well. Forward and backprojection and most of the regularization methods are amenable to parallel processing, and impressive accelerations of the reconstruction are being obtained by implementing these tasks on GPU.

Several methods have been proposed to estimate the regularization hyper-parameters, including obtaining them by imposing a preset spatial resolution, variance, cross-validation error or lesion detection metric, or by using ML estimation (i.e. marginalization over the space of all possible images, which can only be done by introducing approximations). So far, none of these approaches has been widely adopted, probably because they tend to be complicated, they are based on assumptions that are hard to validate and because they do not always produce a degree of regularization that agrees with the preferences of medical experts. In addition, images are often used for multiple tasks (lesion 
detection, activity quantification, kinetic modelling etc.) and task-based optimization will produce a different hyper-parameter value for each task, while for practical reasons, clinicians strongly prefer to work with a single PET image only. This is still an open problem.

Finally, advances in the field of deep learning will certainly also impact PET image reconstruction. One of the current questions is how and where to integrate deep learning methods into the reconstruction process. Possible options include: in the data correction and acquisition part, trying to learn "prior" information applicable in iterative reconstruction from large data sets, or applying deep learning post reconstruction which is further discussed in section 15.

\section{Concluding Remarks}

Because of improvements in the intrinsic and time-of-flight detection resolution and an increasing axial extent, the amount of information acquired by PET systems for the same administered activity and scan time is increasing rapidly. As a result, new approaches to efficiently store and process all these data are needed. The effects caused by inaccuracies in the system model (detector response, geometry, Compton scatter contribution etc.) and by patient motion are having a relatively larger effect on visual quality and quantification accuracy of the reconstructed images. Therefore, there is a need for reconstruction algorithms based on more accurate system models, obtained from efficient calibration procedures or self-calibration, and capable of data driven motion correction. Many regularization techniques for image reconstruction exist and recently new and very effective ones based on deep learning approaches have been proposed, but regularization parameter tuning is still an open problem. 


\title{
11. Motion estimation and correction
}

\author{
Roger Fulton ${ }^{1,2}$, André Kyme ${ }^{3}$
}

1 Department of Medical Physics, Westmead Hospital

2 Sydney School of Health Sciences, The University of Sydney

3 School of Biomedical Engineering, The University of Sydney

\section{Status}

The quantitative accuracy of PET images relies on corrections for physical factors such as photon attenuation, scatter, the partial volume effect and subject motion. Subject motion is arguably the most intractable source of image artefacts and quantitative error in clinical and preclinical imaging. Motion mitigation methods, such as the use of physical supports or restraints, are rarely completely effective. Sedation is used in some cases (e.g. paediatric imaging) but is associated with higher cost and higher risk of complications.

PET motion artefacts typically manifest as blurring of lesions and focal uptake, increased noise, apparent hyper/hypo perfusion defects, and mispositioning, deformation and spill-over or dispersion of activity sources. Thoracic and abdominal scans are mainly impacted by respiratory-induced motion of internal organs and tissues which may prevent detection and accurate characterisation of nodules in the lung, liver, spleen and pancreas. In brain imaging, contrast loss and ghost artefacts are common, especially in paediatric patients and patients with movement disorders and dementia. In preclinical imaging, the motion of unanaesthetised (and unrestrained) laboratory animals precludes the acquisition of usable PET data.

Motion can be classified as non-rigid - exemplified by respiratory and cardiac-induced motion within the chest and abdominal cavities - or rigid - such as gross motion of the head. Motion can be further classified as periodic (e.g. respiratory-related) or random. Periodic motion is counteracted in part using physiological gating in which data are synchronously binned into short frames according to the specific phase of the cardiac or respiratory cycle. Regardless of whether motion is rigid or non-rigid, a key requirement for motion correction is knowing the time course of motion during a scan. Thus, methods to 'track' motion and estimate motion fields have been developed in parallel with correction strategies that utilise these motion data.

\section{Registration-Based Correction}

If PET data have been acquired dynamically or by using gating and then reconstructed as a series of 3D frames, motion can be compensated by choosing one of the frames as a reference and registering all other frames to it, preserving all counts. In this case, the rigid or deformable registration algorithm determines the motion necessary to bring each frame into alignment with the common reference frame. Rigid motion is represented by six degrees of freedom (DoF) comprising the three rotations $R_{x}$, $R_{y}$ and $R_{z}$ about the $x, y$ and $z$ axes, respectively, and the translations $T_{x}, T_{y}$ and $T_{z}$. Non-rigid motion models range from affine transformations (12 parameters) to complex elastic deformations parameterised by many variables. From the co-registered frames, one can obtain more accurate voxelbased time-activity curves for functional images or kinetic modelling (e.g. [109]) (figure 1). Registered frames can also be summed to yield a single motion-corrected frame, thereby reducing noise. As an alternative to conventional 'pairwise' frame-by-frame registration, the registration may also be performed groupwise, where all frame-to-frame transformations are optimised simultaneously 
without the need to explicitly define a reference template. This method has been shown to achieve better motion correction results than conventional frame-by-frame registration in dynamic PET receptor occupancy studies [110]. Despite their flexibility, an important limitation of registrationbased approaches is the inability to correct for intra-frame motion.
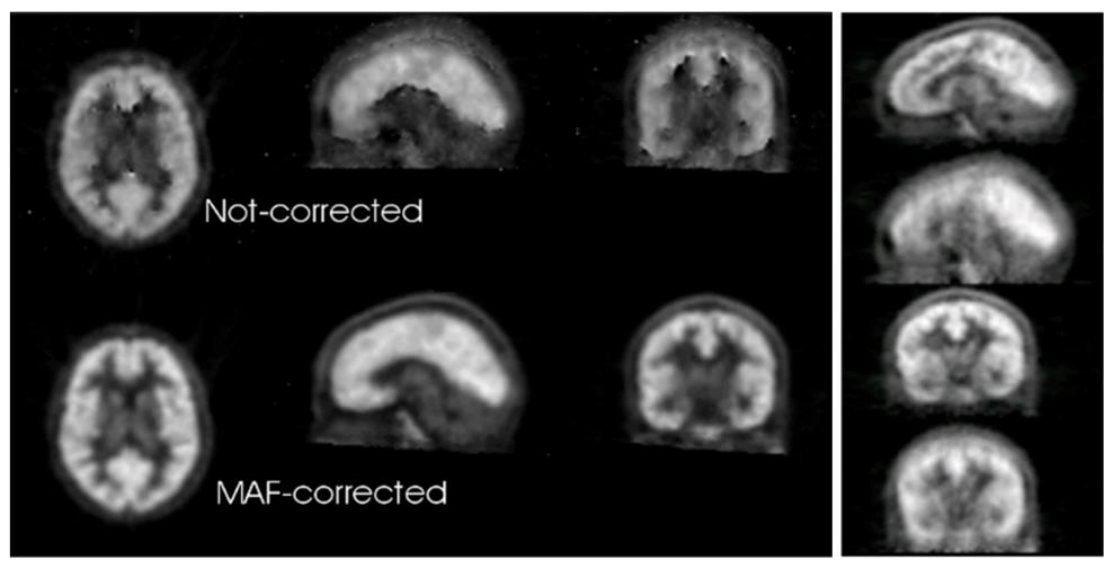

Figure 1. Left panel: Parametric images of F18-altanserin binding potential derived from dynamic PET data [101]. Top row: Without frame-to-frame motion correction. Lower row: With frame-to-frame motion correction showing better correspondence to the expected parameter distribution. Right panel: The same scan motion-corrected with LoR rebinning, shown in sagittal and coronal planes. In each pair the upper image is motioncorrected and the lower one is not.

\section{Line-of-Response Rebinning}

The correction of intra-frame motion requires a more sophisticated approach, line-of-response (LoR) rebinning. Conventionally, list mode coincidence events would be sorted into sinogram projection bins based upon the pair of detectors in coincidence. However, in LoR rebinning, the LoRs are spatially transformed to compensate for motion before being recorded in the sinogram. Time marks in the list mode stream enable coincidence events to be transformed according to contemporaneous pose measurements, typically obtained using an external (usually optical) tracking system. The required transformation for a given LoR is calculated as the change in pose between a reference pose (usually the initial pose) and the pose at the time the event was detected. The sinogram of transformed and correctly normalised coincidence events is then reconstructed to produce a motion-corrected image [111].

One limitation is that some events cannot be assigned to sinogram bins after transformation because they exceed the allowed maximum ring difference, end up outside the field of view, or no longer intersect with a detector pair. Such events are discarded, increasing image noise. This problem is overcome by using list-mode reconstruction which allows all LoRs to contribute to the reconstruction after transformation [112]. LoR rebinning with list mode reconstruction is only suitable for rigid motion and therefore has been applied exclusively to brain PET studies, including in awake small animals [113].

Motion estimation methods for LoR rebinning are typically based on stereo-optical principles (e.g. $[111,114])$. Some of these methods require attached markers or specialised clothing for tracking, others use marker-free techniques to track either sparse features or dense surface meshes using structured light or depth cameras [115] (figure 2). Optical motion tracking cannot directly measure internal deformations and is therefore of limited use for physiological motion, except for detecting respiratory phase using a marker attached to the chest, or indirectly inferring internal motion fields given a suitable external-to-internal motion model [116]. A sufficient motion sampling rate (e.g. $\geq 30$ $\mathrm{Hz}$ ) is particularly important in faster moving subjects and awake rodents. 
In PET/MR hybrid scanners, the use of MRI navigators or tagging pulse sequences, interleaved with normal acquisition, can provide the rigid or non-rigid motion fields needed for motion correction of the PET data [117]. These and other MR-based methods are discussed in section 6 of this roadmap article.

\section{Data-Driven Motion Correction}

Detecting the respiratory phase from raw PET projection data has been reported [118]. However, while it has been shown that data-driven methods can accurately estimate 6 DoF rigid motion parameters in CT head scanning, the much higher noise in raw PET data may, in many cases, be a barrier to obtaining rigid or non-rigid motion parameters with the necessary accuracy and frequency for successful motion-correction. Rather than treating motion estimation and motion correction as independent tasks, joint reconstruction approaches in which the motion and the motion corrected image are simultaneously optimised in an iterative fashion have shown promise in gated PET myocardial perfusion studies [119].
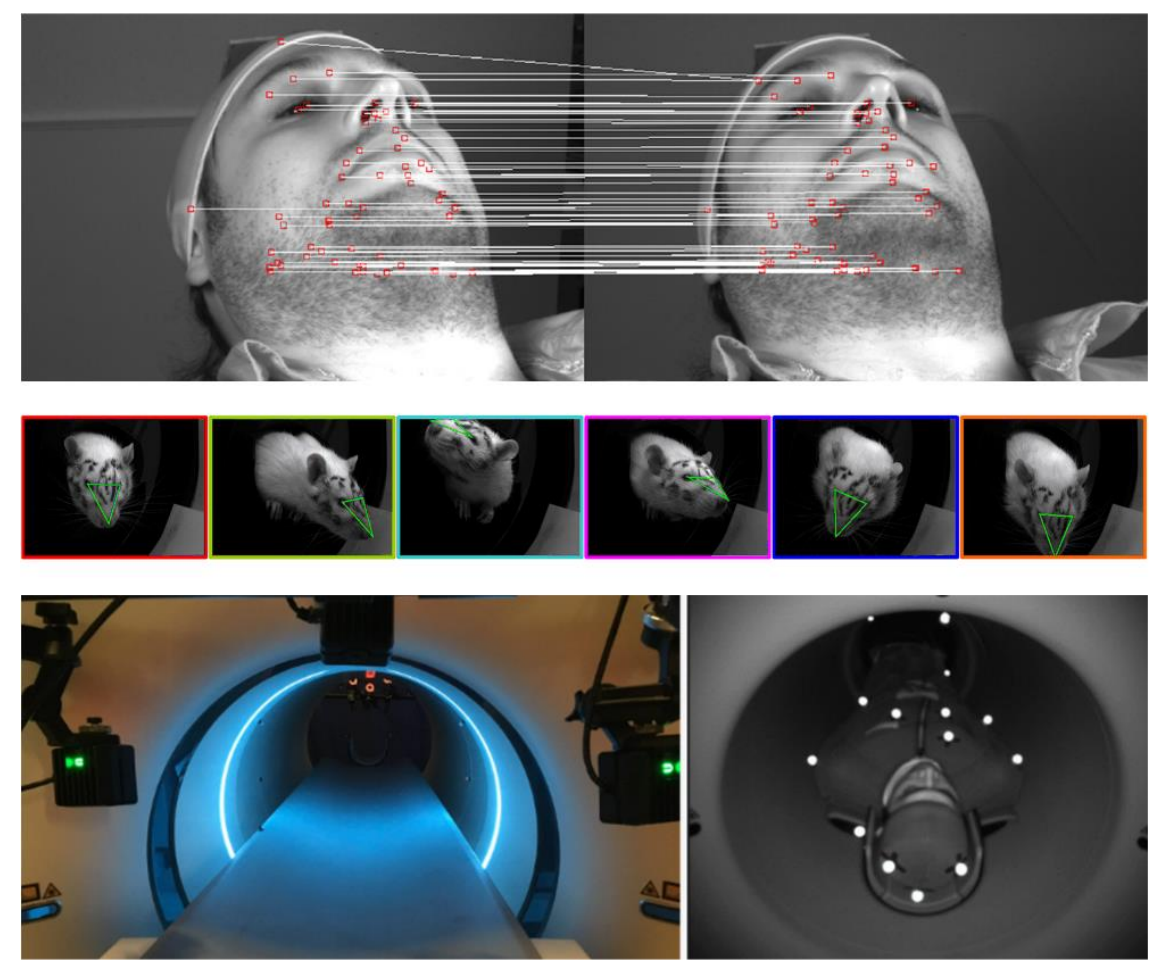

Figure 2. Examples of stereo-optical tracking techniques applied to PET for humans (top row), rats (middle row) and long-bore clinical scanners (bottom row).

\section{Current and Future Challenges}

Despite advances in PET motion correction technology over the past 3 decades, translation to the clinical domain has been slow. Some PET scanner manufacturers offer automated data-driven gating methods for respiratory motion, and methods to correct for head motion in simultaneous PET/MR, but there are no commercially available solutions for rigid head motion correction in PET/CT despite effective in-house methods being the routine in some research centres. Head motion correction appears unlikely to become a routine clinical procedure until it can be performed in a completely automated and reliable fashion without the need for additional patient setup or additional processing time. 
The current trend towards developing long axial-FoV PET scanners [1,2] has important implications for motion correction. For example, these high-sensitivity systems can dramatically reduce scan times and hence the likelihood and impact of involuntary motion. Motion correction in such systems is largely unexplored. It may be necessary, for instance, to track multiple parts of the body at once, overcoming line-of-sight challenges. Motion-corrected time-activity curves obtained from all organs in the body to facilitate new studies of brain-gut signalling and gut biota will also be a completely new motion correction challenge.

Advances in PET instrumentation (e.g. higher resolution, faster detectors and electronics with better ToF capability) and image reconstruction are making PET increasingly susceptible to small amounts of motion. To fully benefit from these advances, the motion estimates supporting motion correction should have an accuracy roughly an order of magnitude better than the target spatial resolution. This is a challenging motion estimation problem, especially for awake rodent brain imaging where the accuracy requirement is $\leq 100 \mu \mathrm{m}$.

\section{Advances in Science and Technology to Meet Challenges}

\section{Non-Rigid Motion Correction}

Further development is needed to improve the accuracy with which gated images can be registered to provide a low-noise motion-corrected image. Phase-matched attenuation maps will be needed for accurate quantification, and these may be generated in the future using MLAA (maximum likelihood activity and attenuation) reconstruction [120] which can generate both activity and attenuation maps from ToF PET data. Improved ToF capability will enable better registration-based correction of nonrigid motion since individual frames can be shorter without degrading SNR. Moreover, a tantalising implication of 10 ps timing resolution [79] will be the ability to perform non-rigid motion correction by shifting the location of individual annihilation events (obtained from direct ToF reconstruction) according to measured motion fields.

In cardiac PET/MR, there is evidence that ECG-based cardiac gating and MR navigator-based respiratory gating together provide better visualization and quantification of tracer uptake in the myocardium than respiratory gating alone [121]. Indeed, PET/MR currently offers the most promising opportunities for applying non-rigid motion correction to PET images because of its ability to elucidate internal motion from simultaneously acquired MRI data.

\section{Rigid Motion Correction}

The lack of commercially available solutions for motion-corrected PET imaging of the head and brain is an indication that the successful implementation of effective techniques and algorithms in research environments does not guarantee their translation to the clinic. Translation may be encouraged by the development of optical head tracking technologies that rely only on natural surface features without attached markers, are reliable, completely automated, and fully integrated into the scanner. This degree of integration and automation can ultimately only be realised through collaborative development with scanner manufacturers.

In all of these future developments we foresee that deep neural network-based models could play an important role [122]. Neural networks could augment or replace the feature detection and/or pose 
estimation steps in traditional stereo-vision pipelines; neural network-based image registration may allow improved alignment of noisy gated images; and image-based data-driven correction of motion artefacts may be feasible with supervised neural networks given sufficiently large labelled training sets sampling the multi-dimensional motion space. Models that are generalisable across different subjects, scanners and protocols will be vital for both practicality and acceptance.

\section{Concluding Remarks}

In clinical PET imaging, effective motion correction methods are essential for accurate image quantification and diagnosis. They are also essential for imaging awake, unrestrained laboratory animals. Enormous advances have been made in the development of such methods over the last thirty years. Nevertheless, commercially available solutions are rare and yet to enter the mainstream. Achieving accuracy, automation, speed and reliability will be key to the future routine deployment of motion estimation and correction methods in clinical PET imaging. 


\section{Kinetic Modelling and Parametric Imaging}

\section{Cristina Lois ${ }^{1}$, Hasan Sari ${ }^{2,3}$, Julie Price ${ }^{2,3}$}

1 Gordon Center for Medical Imaging, Massachusetts General Hospital \& Harvard Medical School, Boston, MA, USA

2 Department of Radiology, Massachusetts General Hospital \& Harvard Medical School, Boston, MA, USA

3 Athinoula A. Martinos Center, Massachusetts General Hospital \& Harvard Medical School, Boston, MA, USA

\section{Status}

Kinetic modelling and parametric imaging have renewed potential in view of recent PET imaging advances that include rational radiotracer and radioligand design, instrumentation technology, and computational hardware and software developments.

The evaluation of a novel radiotracer is best accomplished using arterial input function (IF) based compartmental modelling methods (e.g., 1- or 2-tissue, 1T or 2T) that enable careful evaluation of the radiotracer kinetics in blood and tissue. For brain imaging, these methods also enable verification of a reference region ( $\mathrm{ROI}_{\mathrm{REF}}$ ) that lacks specific uptake/binding. The $\mathrm{ROI}_{\mathrm{REF}}$ is representative of nondisplaceable (ND) tissue uptake and used to quantify specific binding, i.e., $V_{T} / V_{N D}=D V R$ (distribution volume ratio) and $B P_{N D}=D V R-1$ (binding potential), where $V_{T}$ and $V_{N D}$ are the volumes of distribution of total radiotracer in a target region and of non-displaceable uptake, respectively. For cases when imaging targets are distributed throughout brain, such that $\mathrm{ROI}_{\mathrm{REF}}$ cannot be readily identified (e.g., TSPO or NET), compartmental modelling may be desirable for a subset of subjects to validate a simplified pseudo-reference region approach. A data-driven hybrid deconvolution approach (HYDECA) was recently proposed for the global determination of $\mathrm{V}_{\mathrm{ND}}$ using singular value decomposition to estimate the impulse response function for several regional time-activity curves (TAC), but knowledge of the metabolite-corrected arterial IF was still required [123]. Accordingly, image-derived input functions (IDIFs) remain long sought-after alternatives, as discussed below.

Non-compartmental methods include spectral analysis and linear graphical methods such as Patlak and Logan. Linear analysis alternatives are strongly leveraged for direct parametric image reconstruction, assuming steady-state conditions are established. For brain imaging, reference tissue based alternatives (model-based and non-model based) are widely applied, assuming a robust ROI REF can be identified. There are several well-known considerations for these simplified methods that relate to data variability and noise. The Simplified Reference Tissue Model (SRTM) is based on 1T model kinetics but is often applied to data that can be better described with additional model parameters. The Logan analysis is particularly vulnerable to noise-induced bias. Several alternate methodologies were developed to mitigate this bias for regional and voxel level analyses, including the Multilinear Reference Tissue Models (e.g., MRTM, MRTM2) and approaches that invoke data smoothing and/or spatial-temporal constraints, as recently reviewed [3,124] (Figure 1). These methods have been modified and expanded to enable in vivo assessment of endogenous neurotransmitter levels (time-invariant models) and to assess alterations in neurotransmitter levels induced by pharmacological or behavioural challenges (models with time-varying terms, e.g., IpntPET) [125]. The latter methods have been applied in simultaneous PET/MR imaging to quantify dynamic receptor occupancies with PET and dynamic neurovascular changes with $\mathrm{FMRI}$. 
Kinetic modelling and parametric imaging are vulnerable to noise and bias, particularly when PET kinetics are inconsistent with methodological assumptions. The optimal kinetic method is based on knowledge and trade-offs between chemical and physiological characteristics of the radiotracer and process-of-interest over time and overall model performance [3,124]. Parametric images are commonly generated from reconstructed dynamic data (indirect) and often necessitate a denoising step prior to kinetic analysis to improve the accuracy and reliability of parameter estimates. Several denoising techniques have been proposed for dynamic PET, including non-local means (NLM), which exploits self-similarities in images by comparing intensity differences in local neighbourhoods, and HighlY constrained backPRojection (HYPR), which capitalizes on the spatiotemporal correlation existing in a dynamic acquisition [126]. Powerful direct 4D parametric reconstructions at the sinogram level, where noise is more accurately modelled, are increasingly feasible. Computational advances enable approaches that decouple image reconstruction and kinetic modelling and allow use of nonlinear models $[3,127]$. Coupling of greater sensitivity and spatial resolution improves quantification and mapping of temporal and spatial dynamics and simultaneous parameter estimation.

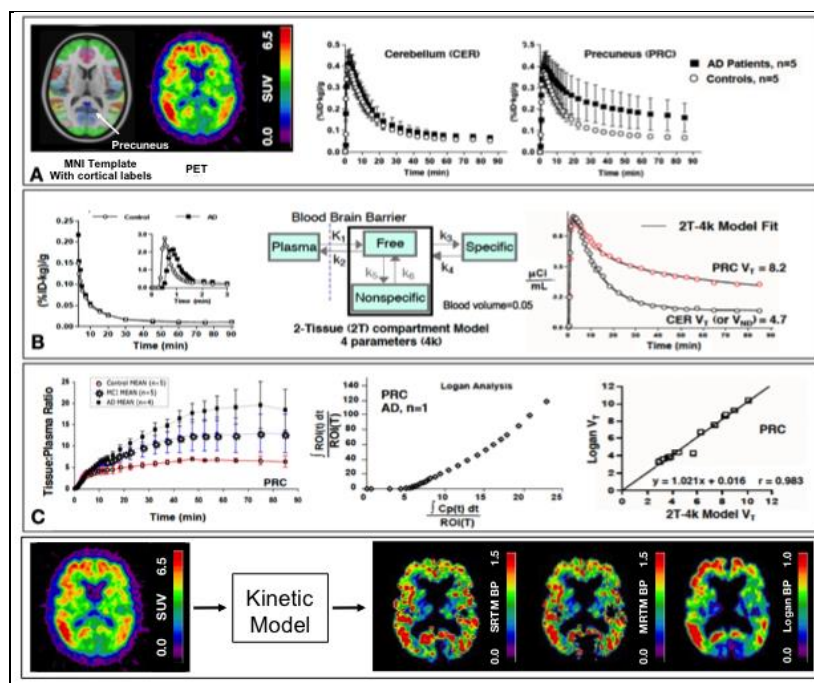

Figure 1. Kinetic analysis of dynamic ${ }^{11} \mathrm{C}-\mathrm{PiB}$ PET radioligandprotein binding studies in controls and patients with mild cognitive impairment $(\mathrm{MCl})$ and Alzheimer's Disease (AD).

(A) Left: MRI template and Harvard-Oxford atlas labels with coregistered PET SUV $40-70$ (MCl: 74 years); Right: Average TACs consistent with reversible binding (target: precuneus, PRC) and non-displaceable uptake (reference: cerbellum, CER).
(B) Left: Metabolite-corrected arterial plasma IF (unmetabolized percentage: $86 \%, 12 \%$, and $6 \%$ at 2, 30, and $90 \mathrm{~min}$, respectively); Middle: $2 \mathrm{~T}-4 \mathrm{k}$ compartmental model used to assess BBB transport and kinetics of free+nonspecific (ND) and specific radioligand binding; Right: 2T-4k model fits and distribution volume outcomes for target $\left(\mathrm{V}_{\mathrm{T}}\right)$ and reference $\left(\mathrm{V}_{\mathrm{ND}}\right)$.

(C) Left: Tissue:plasma ratios reveal eventual plateau during study, across groups consistent with transient equilibrium and eventual linearity of the Logan graphical plot (Middle); Right: Agreement between nonlinear compartmental and linear graphical $V_{T}$ values was verified. (D) Left: PET SUV image; Right: Parametric binding potential $\left(\mathrm{BP}_{\mathrm{ND}}\right)$ images determined by reference-tissue modelling methods show good correspondence for ${ }^{11} \mathrm{C}$ $\mathrm{PiB}$, despite voxel noise and some violation of assumptions (i.e., SRTM based on $1 \mathrm{~T}$ model but ${ }^{11} \mathrm{C}-\mathrm{PiB}$ data fit by $2 \mathrm{~T}$ model, Logan violations of least-squares assumptions, and methods may become unstable when kinetics in reference and target regions are similar). Data processing algorithms and advanced methodologies can help mitigate unwanted bias. See text and references $[3,124]$ for definition of abbreviations and detail.

\section{Current and Future Challenges}

Fully dynamic PET generally requires $\geq 60$ min data acquisition divided into short time frames for image reconstruction. Frame number and duration should be optimized as a trade-off between sufficient TAC temporal resolution and counting statistics, although scatter estimation can be challenging for short frames. Individual frames are generally independently reconstructed and insufficient statistics yield noisy images that could result from insufficient noise regularization, inadequate modelling of the noise distribution, or noise-induced bias. Subject motion during imaging is also a great challenge and results in image blurring and mismatch between the attenuation map and PET emission data. The accuracy of motion correction depends on the method used, the radiotracer 
spatiotemporal distribution, and the organ under study. Simple post-reconstruction frame-by-frame alignment is often used in brain imaging but cannot correct intra-frame motion nor quantitative inaccuracies created by attenuation mismatch. Respiratory, cardiac, or bowel motion require more advanced corrections (see Section 11). Partial volume corrections, also with well-known complexities, can be difficult to apply well across health and disease. See topical reviews $[3,127]$.

Input function determination traditionally requires arterial catheterization, blood sampling and plasma assays, and correction for radiolabelled metabolites for many radiotracers. This is labour intensive and complicates subject participation, data acquisition and analysis. Image-derived input functions (IDIFs) are long sought-after alternatives based on TACs derived from blood vessels. IDIFs have worked successfully in cardiac and whole-body imaging where large blood pools are available but are more challenging in brain because of small vessel sizes and partial volume effects. There is also potential to miss the early arterial IF peak if PET sampling frequency is insufficient. Population-based IFs and metabolite curves may be an alternative but may not fully capture individual differences across subjects. Alternative methods offer simultaneous estimation of analytical IF forms with kinetic parameters when fitting several regional TACs (e.g., simultaneous estimation or SIME approach), but often require at least one blood sample for scaling, and require validation for each tracer [3,128]. IDIF methods are also vulnerable to subject motion.

Desirable radiotracer characteristics include high selectivity and specificity, high bioavailability and low plasma protein binding, limited or measurable metabolism, low but measurable nonspecific uptake, and appropriate lipophilicity for sufficient BBB penetration (brain imaging) $[129,130]$. Associated challenges include: difficulty to adequately capture radiotracer dynamics, transient equilibrium and/or steady-state during the imaging period; difficulty to reliably determine a metabolite-corrected IF when in vivo radiotracer metabolism is very rapid; difficult identification of an optimal model or non-compartmental approach if controls and patients exhibit different kinetics during the study (e.g., reversible vs. irreversible). Reliable quantification is also challenging if nondisplaceable, off-target, or background uptake interferes with target quantification or if such measures are low, difficult to obtain, variable, or ill-suited for image normalization or reference-tissue modelling. Tissue heterogeneity can also be challenging (e.g., mixture of differing grey and white matter kinetics, non-uniform sub-voxel protein localization, and tumour heterogeneity).

Evaluation of model performance on regional- or voxel-basis can be complicated. Accordingly, latescan standardized uptake values (SUV, normalized to injected dose and body mass) and tissue ratios ( $S U V R=S U V_{\text {target }} / S U V_{\text {REF }}$ or SUR=SUV Tumour $/ S U V_{\text {Blood-pool }}$ ) are widely used in clinical research because of short acquisitions, computational ease, and reduced variability of image-based ratios that can yield greater statistical power for the detection of group differences and longitudinal change. The SUVR, however, is a surrogate measure of radiotracer $V_{T}$ (the tissue:blood concentration ratio at equilibrium) that is vulnerable to significant bias, particularly after bolus injection of reversibly-binding radiotracers when equilibrium assumptions are violated $[3,124]$. A major challenge is further demonstrating the importance of quantitative PET in the context of what is lost and gained by simplified alternatives and the usefulness of regional and parametric alternatives [131]. As we continue to struggle with variable radiotracer kinetics, signal-to-noise, and reference region performance, while reducing imaging times to lessen participant study burden, we look forward to further advances over the next decade that will provide a new quantitative landscape for in vivo imaging. 


\section{Advances in Science and Technology to Meet Challenges}

Recent PET instrumentation advances improve the quantitative accuracy of PET. Developments in digital PET technology and detector design have significantly improved temporal and spatial resolution capabilities and sensitivity. These advancements, coupled with longer axial field-of-views (aFOV), innovative bed-motion technology, and cumulative progress in image reconstruction techniques (see section 10), have demonstrated the feasibility of indirect and direct whole-body parametric imaging and clear improvements realized by the latter [127] (figure 2). The Explorer consortium combined state-of-the-art technologies with 140-cm (PennPET) and 194-cm (uEXPLORER) aFOVs allowing singleacquisition body imaging at ultra-high sensitivity and ultra-short frame durations for pharmacokinetic analysis (as short as $1 \mathrm{~s}$ [43]). These innovations greatly advance dynamic imaging capabilities (see Section 8) beyond traditional model configurations by allowing for more accurate modelling and quantification of the vascular dynamics of radiotracer delivery, of radiotracer heterogeneity in a given tumour or tissue type, and of varying kinetics in different tissues throughout the body (fast and slow, early and late). Total-body scanners will also allow use of an IDIF from aorta or left ventricle in neuroimaging studies. From a modelling perspective, these advancements will improve microparameter identifiability, macro-parameter reliability, and model selection.
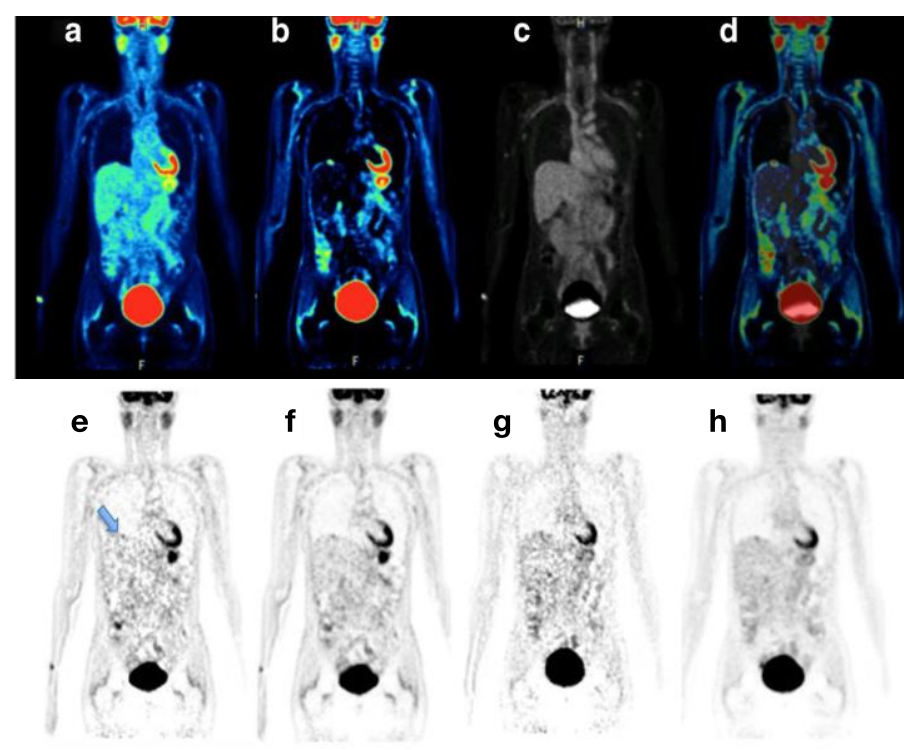

Figure 2. Dynamic whole-body PET imaging (adapted from [127]). TOP: Comparison between (a) SUV images (70-90 min post FDG injection), (b) parametric images (0-90 $\mathrm{min}$ ) of $K_{i}$ and (c) $V_{E}$ generated from a FDG scan using the Patlak method with an image-derived input function and a linear regression with spatial constraints. (d) Fusion of $\mathrm{K}_{\mathrm{i}}$ and $\mathrm{V}_{E}$ images. BOTTOM: Parametric images for $30 \min (6 \times$ $5 \mathrm{~min} /$ pass) time windows. (e) Indirect $K_{i}$ and (f) direct $K_{i}$ images for frames spanning $\sim 10-40$ min post-injection. (g) Indirect $\mathrm{K}_{\mathrm{i}}$ and (h) direct $\mathrm{K}_{\mathrm{i}}$ images for frames spanning $\sim 60-90 \mathrm{~min}$ post-injection. Three iterations (21 subsets) were used, and $6 \mathrm{~mm}$ Gaussian filter postsmoothing applied. The small tumour (shown by arrow) at the dome of the liver is seen in early imaging $(e, f)$ but only on direct $\mathrm{K}_{\mathrm{i}}$ image for later imaging (h). [Ki: net FDG influx; VE: exchangeable volume of distribution]

Radiotracer development efforts (see section 5) also address challenges using sophisticated methods during design to optimize in vivo radiotracer performance with feedback from kinetic modelling. Academic, industry and academic/industry partnership efforts are aimed to provide improved radiotracers for existing targets-of-interest and/or new radiotracers for imaging of innovative targets and drug discovery $[124,129,130]$.

Robust imaging of early bolus-injection IF dynamics in carotid arteries less impacted by partial volume or use of left ventricle data in total-body PET would reduce burden of IF determination dramatically. New data sharing plans to enable availability of large PET datasets including processed blood (and metabolite) data could strengthen population-based IDIF approaches [132]. Automated motion correction methods that fully address attenuation mismatch issues are essential to improve quantitative accuracy for robust modelling measures and parametric images. 
Artificial intelligence (see section 15) is also addressing challenges related to kinetic modelling such as data corrections (e.g., motion correction, attenuation correction in PET/MR), image reconstruction (e.g., noise regularization, image deblurring), and post-processing. Furthermore, Al applied to kinetic modelling has shown promise [133].

Overall these advancements enable more accurate quantification with better mapping of tissue and/or tumour heterogeneity, use of lower injected doses that enable repeated imaging at shorter intervals and imaging of multiple targets for more comprehensive patient evaluations. Using Alzheimer's disease as an example, advanced quantification and parametric imaging could provide future in vivo imaging results that are more comparable to neuropathological evaluations and improve assessment of patient status. This includes the detection of very low levels of tau deposition in a small area of entorhinal cortex that is currently complicated by off-target and extra-cerebral uptake, or disentanglement of the spatiotemporal characteristics of multimodal intra-subject images of neuropathological protein accumulation (e.g., specific forms of tau/tangles, amyloid-beta, and alphasynuclein). These advances can impact basic science and clinical research, drug discovery and development, and conduct of clinical trials. All leading to improved detection of early disease and/or therapeutic efficacy.

\section{Concluding Remarks}

Recent advances in tomograph technology, radiotracer development, and computational methods are meeting the challenges that limit the usability of quantitative PET in clinical applications. It is essential that we achieve the capacity for sensitive detection of earliest disease and therapeutic responses. A major challenge now is recognizing the importance of quantitative PET imaging and understanding how best to leverage these advances to better serve the community.

\section{Acknowledgements}

The authors acknowledge funding support from R01AG052414, R01AG050436, and R01DK112700. We also thank Profs. S. Meikle and T. Jones for their helpful comments. 


\section{Statistical reliability, reproducibility and standardisation of quantitative PET}

Ronald Boellaard ${ }^{1}$, Robert Jeraj ${ }^{2,3}$

1 Radiology and Nuclear Medicine, Cancer Center Amsterdam, Amsterdam University Medical Center, location VUMC

2 Departments of Medical Physics, Human Oncology and Radiology, University of Wisconsin

3 Faculty of Mathematics and Physics, University of Ljubljana

\section{Status}

Positron emission tomography (PET) plays an important role in the diagnosis, prognosis, treatment prediction and treatment response assessment of patients suffering from cancer, cardiac, neurological and other diseases. PET allows quantitative assessment of radiotracer distribution in vivo and quantitative reads are being more frequently used in clinical trials and in the clinic. However, like many other types of medical measurements, PET also suffers from various sources of uncertainty causing both bias and variability in radiotracer uptake quantification [7]. Sources of uncertainty can be classified as technical and biological factors as well as factors related to acquisition and image reconstruction settings [7]. Moreover, choices made for image analysis, such as tumour or region of interest delineation methods and extracted quantitative metrics, affect the accuracy and precision of quantitative PET reads. An example is given in Figure 1 showing that PET images reconstructed using different settings can have a large impact on several quantitative metrics derived from these images. Quantitative assessment in oncology FDG PET/CT studies can easily differ by a factor of 2 depending on the imaging procedures, settings applied and type of image analytics. To reduce variability of quantitative PET, guidelines have been published providing recommendations for various steps involved in the imaging procedures, such as: (1) patient preparation; (2) PET acquisition; (3) image reconstruction; (4) image analysis and quantitative reads and; (5) interpretation of images and quantitative results [134]. Apart from recommendations on how to perform the PET/CT examination, a proper harmonisation of the PET system performance is essential to obtain quantitative reads that are reproducible, i.e. comparable between different scanners and imaging sites. To this end accreditation programs, such as the ones by the Society of Nuclear Medicine (SNM) Clinical Trials Network(CTN) and European Association of Nuclear Medicine (EANM)/EARL, have been initiated allowing sites to align the performance of their PET/CT systems with common standards. The progress of these programs were recently reported $[135,136]$ showing the feasibility of running these programs successfully and sustainably. 


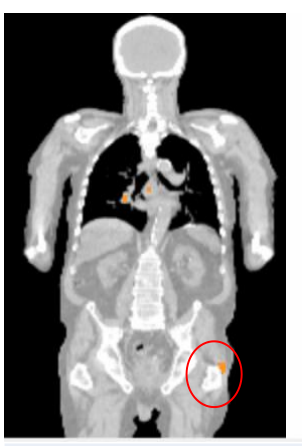

Parameter/Reconstruction SUVmax

Metabolic active tumor volume

Total lesion glycolysis

Entropy (heterogeneity)

Dissimilarity (heterogeneity)

Compactness (morphology)

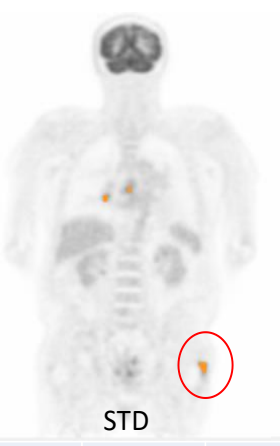

Standard High resolution Difference (\%)

\begin{tabular}{|l|l|r|}
\hline 6.81 & 10.34 & 51.73 \\
\hline
\end{tabular}

\begin{tabular}{|l|l|r|}
\hline 9.15 & 7.66 & -16.35 \\
\hline
\end{tabular}

\begin{tabular}{l|l|l|l|}
\hline 40.12 & 44.75 & 11.53
\end{tabular}

\begin{tabular}{|l|l|l|}
\hline 5.41 & 5.49 & 1.38 \\
\hline
\end{tabular}

\begin{tabular}{|l|l|r|}
\hline 17.95 & 13.93 & -22.39 \\
\hline
\end{tabular}

\begin{tabular}{l|l|l|l|l}
0.59 & 0.49 & -18.14
\end{tabular}
Figure 1. Impact of variation in PET image quality on several quantitative standard and radiomic parameters. Metrics were extracted using a $50 \%$ of SUV $_{\text {peak }}$ isocontour. Images and data are presented for a standardised (STD=EARL compliant) and a high resolution reconstruction.

\section{Current and Future Challenges}

Many of the technical and biological sources of uncertainty do not depend on the PET technology used to perform the PET examination. For example, synchronisation of clocks of all devices measuring radioactivity or activity concentrations, including the PET systems and dose calibrators (technical factor) should always be properly taken care of. Similarly, tracer uptake time (biological factor) should be chosen and standardised based on tracer kinetics, e.g. for FDG typically set at 60 min post injection. On the other hand, there are advances in PET technology that affect image quality and quantification. For example, improved time of flight performance by using digital PET technology, use of smaller detectors/scintillation crystals and introduction of resolution modelling during image reconstruction, so called point spread function (PSF) reconstruction, led to PET images with higher spatial resolution and increased contrast-to-noise ratio (CNR). These technological improvements enhance the diagnostic quality of the images and allow reduction of the amount of radioactivity administered. At the same time, the use of these new technologies also affects quantitative performance of PET systems. Readers observed that after installation of a new PET/CT system standardised uptake values (SUV) could change by a factor of 2 and/or they observed a change in visual scoring of FDG uptake [137]. Consequently, these large quantitative changes have a detrimental effect on the reproducibility of quantitative results either with historical cohorts or between PET systems operating at different levels of technology, particularly a challenge in multicentre studies.

A possible solution is simply to keep on using current PET/CT performance standards. This implies that each PET system is equipped with an acquisition and reconstruction protocol that complies with a standard based on somewhat outdated technology. Indeed, many imaging sites opt to perform two different PET image reconstructions, one that complies with harmonizing performance standards and a second one optimised for clinical use. More recently, efforts have been undertaken to adapt existing standards to new ones that better fit state of the art PET technology, thereby attempting to bridge the gap between quantitative performance and visual attractiveness of the images. Another possible approach that combines unbiased quantitative accuracy for SUV measurements, harmonizing performance and visual attractiveness of the images, is based on adaptive denoising [138]. This approach only requires reconstructing one dataset that can be used for quantitation and visual interpretation at the same time. 
The main challenge of moving from an existing standard to a new (improved) standard is that both quantitative and diagnostic visual reads will change substantially with possible effects on patient management [137]. It is therefore of utmost importance that not only the standards are updated, but that quantitative and visual interpretation criteria are adapted at the same time.

Other interesting developments are the use of many new radiotracers and isotopes, such as radiotracers labelled with ${ }^{89} \mathrm{Zr}$ and ${ }^{68} \mathrm{Ga}$. For these radiotracers and/or isotopes, imaging guidelines and PET standards need to be developed. Recent studies suggest that current PET/CT system performance standards, implemented for FDG, can also accommodate ${ }^{89} \mathrm{Zr}$ and ${ }^{68} \mathrm{Ga}$ labelled tracers by only verification of the PET system calibration (for these isotopes). Use of harmonizing standards for FDG seems to allow harmonisation for other isotopes as well [135]. The main advantage for sites is that they only need to implement a single harmonised image reconstruction protocol for all their studies.

Finally, most standards to date focus on the use of PET in oncology. In neurology, many brain PET studies are performed while a PET system performance standard is not yet widely implemented. Efforts are being undertaken by the Quantitative Imaging Biomarker Alliance (QIBA) and EARL and those initiatives will likely improve the reproducibility of quantitative brain PET in multicentre studies. Many other new programs for specific clinical applications of PET, such as in cardiology, will likely arise in the future as well.

\section{Advances in Science and Technology to Meet Challenges}

Apart from harmonizing image quality and quantification to enhance (multicentre) reproducibility, precision of PET reads are equally important. Both early and late response assessment using FDG PET in oncology is an important clinical tool for treatment response prediction and/or to assess drug efficacy in trials. The variability of FDG uptake shows a test-retest variability of 10 to $15 \%$ leading to confidence intervals of about $30 \%$ for tumours having a maximum SUV $>4$. The confidence intervals vary by the type of quantitative metrics extracted from images (e.g., different SUV metrics), and can dramatically increase in radiomics analyses [139]. The variability in SUV is caused by e.g. uncertainties in injected activities, biological variabilities and by statistical noise. To mitigate some of these effects the maximum SUV (SUV $\max$ ) can be replaced by a so-called $S U V_{\text {peak. }}$. The latter represents the mean SUV in a $1 \mathrm{~mL}$ spherical volume of interest positioned such to yield the highest value across all locations within the tumour, although one should be aware that different definitions of SUV $V_{\text {peak }}$ can lead to significantly different quantitative results. The variability related to statistical noise is inversely proportional to the number of detected counts. To keep the noise level as similar as possible among patients, the injected activity and the acquisition time per bed position can be adjusted as a function of body weight [134] or considering patient-specific attenuation for different anatomical regions, the injected activity and the detection sensitivity of the PET scanner [138].

Of particular importance to increase quantitative accuracy of PET imaging is consistent and, particularly in the multicentral clinical trial setting, centralized image analytics, which has been shown to lead to significantly lower variability of the quantitative PET evaluation [140]. Another significant source of quantitative uncertainty, particularly in oncology, is definition of target volumes. In general, auto-segmentation algorithms are more reproducible than manual segmentation, and advanced 
image analysis paradigms provide generally more accurate segmentation than approaches based on PET activity thresholds, which are still most commonly used [141].

PET systems with an extended axial field of view have gained recent interest, as discussed in section 8. These so-called total body PET/CT systems are designed to have an axial coverage of $70 \mathrm{~cm}$ or more. In fact, the first total body PET system of almost $2 \mathrm{~m}$ axial length has been installed and first clinical results have been published [1]. These new systems show an enormous increase in sensitivity compared to state of the art (digital) PET/CT systems. The excellent sensitivity enhances CNR allowing to reduce scan duration and/or administered radiotracer activity or perform the examination at very prolonged uptake times. Imaging guidelines will need to be further developed and adapted to these new systems. Moreover, covering the entire body allows verification of the nett administered activity corrected for renal excretion and the use of an image derived total body activity to normalize SUV may mitigate some of the uncertainties associated with tracer administration and scanner calibration.

Finally, artificial intelligence (Al) offers several new opportunities, such as improvement of image quality to reduce radiation dose and/or to increase CNR to enhance visual reads, but possibly also to harmonize quantitative reads. For example, when the Al model is trained to recognize or characterize image quality and its quantitative performance, it may also be able to harmonize the quantitative reads, i.e. the Al model will provide a harmonised quantitative read regardless of underlying image quality. Future research will inform to what extent such an approach is feasible and accurate. Moreover, Al may be applied to directly interpret images and provide information on diagnosis, prognosis or prediction [10].

\section{Concluding Remarks}

To use PET as a quantitative imaging biomarker, it is essential that quantitative reads are repeatable and reproducible. To this end harmonisation of PET/CT imaging procedures and system performances, together with the harmonized and centralized image analytics in multicentre clinical trial settings, are of utmost importance in order to mitigate the effects of various sources of uncertainty. Moreover, new technological developments, such as digital detectors, new reconstruction methods and systems with long axial fields of view and artificial intelligence approaches increase sensitivity and image quality, thereby improving statistical reliability.

\section{Acknowledgements}

Ronald Boellaard is an unpaid member of the EARL steering board as scientific advisor and chair of the accreditation program. 


\section{Section 14. Dosimetry, Treatment Planning \& Monitoring Response}

Dale L Bailey ${ }^{1,2,3}$, Enid Eslick ${ }^{1,2}$ Kathy P Willowson ${ }^{1,3}$

1 Department of Nuclear Medicine, Royal North Shore Hospital

2 Faculty of Medicine and Health, The University of Sydney

3 Faculty of Science, The University of Sydney

\section{Status}

One of the strengths of functional imaging is its ability to make quantitative measurements of important biological parameters, such as glucose metabolism. Positron Emission Tomography (PET) has always had this quantitative capability, notwithstanding the limitations of finite spatial and temporal resolution. Recently, Single Photon Emission Computed Tomography (SPECT) has become similarly quantitative [142]. Together, these two modalities are important in the field of diagnosis and therapy using radiopharmaceuticals as both have roles in demonstrating radiopharmaceutical targeting for treatment planning, in assessing the radiation dose delivered to diseased and normal tissues, and in the longitudinal monitoring of response to therapy (figure 1).

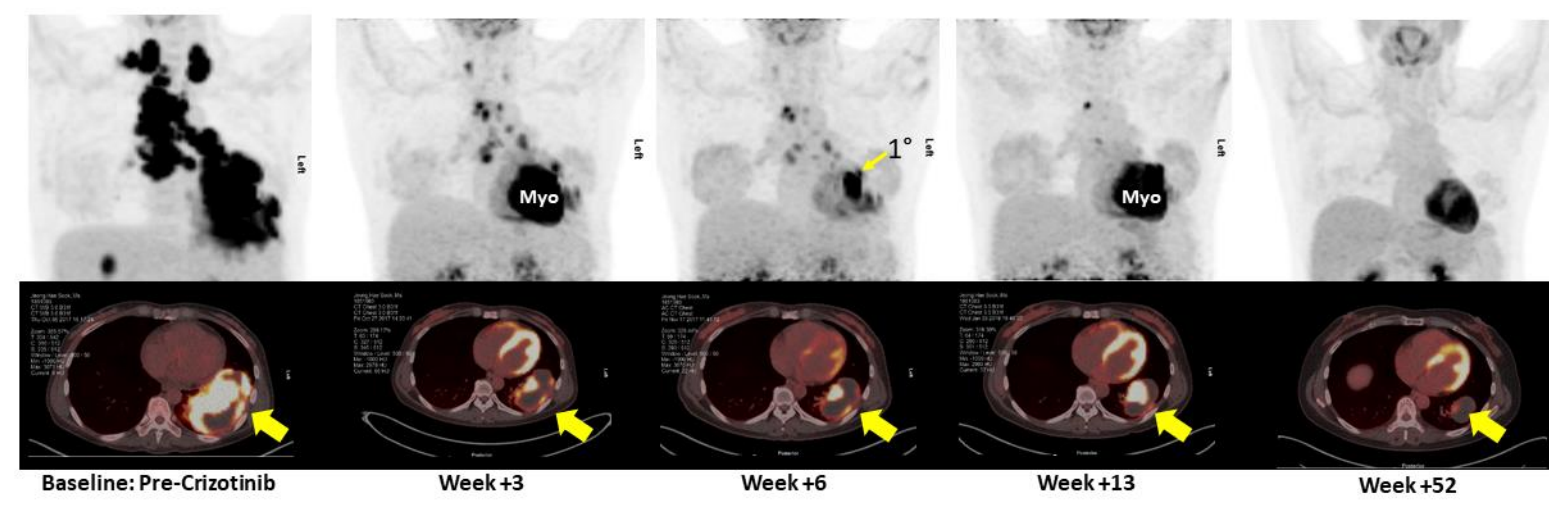

Figure 1. An example of the use of metabolic functional imaging to monitor response to adjuvant pharmacotherapy in a subject with Non-Small Cell Lung Cancer (NSCLC) expressing the anaplastic lymphoma kinase (ALK) gene, and thus suitable for treatment with a protein kinase inhibitor. The post-baseline FDG PET/CT scans were performed regularly using a "lowdose" protocol after commencing therapy. A rapid response is seen on the PET images by 3 weeks, which endures to the one year time point. The subject remains alive and virtually disease-free at the time of writing, some 2.5 years after diagnosis of a very aggressive and advanced Stage IV cancer.

The emergent field of theranostics, which uses the same (or very similar) molecule to interrogate cancerous tissues for therapy planning and then to deliver the therapy loaded up with a radioactive $\alpha$ or $\beta$ - emitter, increasingly relies on quantitative PET and SPECT in an attempt to develop a personalised treatment approach for each individual patient. Table 1 lists the characteristics of the main radionuclides of relevance today in theranostics and dosimetry, and in which role they contribute.

\section{Current and Future Challenges}

Quantitative PET has become an indispensable tool in the management of many diseases, but particularly in cancer management. The major strengths that it has for this area are its whole body as opposed to limited regional - evaluation (i.e., nodal and metastases staging), high contrast for target:normal tissue, and quantitative capability to initially stage disease and measure functional response to treatment or progression, rather than relying on change in size of a lesion. 
The role of PET in treatment planning and dosimetry is reasonably limited at present, in general, due to the radionuclides most commonly used in PET. The half-lives of ${ }^{18} \mathrm{~F}$ and ${ }^{68} \mathrm{Ga}$ (table 1 ) are simply too short to match the biological processes involved when it comes to using long-lived therapeutic radionuclides. Copper-64, ${ }^{89} \mathrm{Zr}$ and ${ }^{124} \mathrm{I}$ are PET tracers with longer half-lives that do allow PET imaging over an extended period of time, although in the case of ${ }^{64} \mathrm{Cu}\left(\mathrm{t}_{/ 2}=12.7 \mathrm{~h}\right)$ it is probably limited to a maximum of $24 \mathrm{hrs}$ post-injection on current state-of-the-art equipment.

Table 1 - Radionuclides of interest in quantitative imaging related to oncology

\begin{tabular}{|c|c|c|c|c|}
\hline Radionuclide & Half-life & SPECT or PET & Emission & Main Role in Management \\
\hline Fluorine-18 & $109.5 \mathrm{~m}$ & PET & $\beta^{+}$ & Initial staging and response assessment \\
\hline Phosphorous-32 & $14.2 \mathrm{~d}$ & SPECT $($ Brem*) & $\beta^{-}$ & Therapy \\
\hline Copper-64 & $12.7 \mathrm{~h}$ & PET & $\beta^{+}$ & Initial staging and response assessment \\
\hline Copper-67 & $61.8 \mathrm{~h}$ & SPECT & $\beta^{-}$and $\gamma$ & Therapy \& imaging for dosimetry \\
\hline Gallium-67 & $78 \mathrm{~h}$ & SPECT & $\gamma$ & Treatment planning \\
\hline Gallium-68 & $68 \mathrm{~m}$ & PET & $\beta^{+}$ & Initial staging and response assessment \\
\hline Zirconium-89 & $3.3 \mathrm{~d}$ & PET & $\beta^{+}$ & Treatment planning \\
\hline Yttrium-90 & $64 \mathrm{~h}$ & PET or SPECT $($ Brem*) & $\beta^{-}$and $\beta^{+}$ & Therapy \& imaging for dosimetry \\
\hline Technetium-99m & $6 \mathrm{~h}$ & SPECT & $\gamma$ & Initial staging and response assessment \\
\hline Indium-111 & $67.4 \mathrm{~h}$ & SPECT & $\gamma$ & Treatment planning \\
\hline lodine-123 & $13 \mathrm{~h}$ & SPECT & $\gamma$ & Initial staging and response assessment \\
\hline lodine-124 & $4.2 \mathrm{~d}$ & PET & $\beta^{+}$ & $\begin{array}{l}\text { Initial staging, response assessment, } \\
\text { therapy \& imaging for dosimetry }\end{array}$ \\
\hline lodine-131 & $8 d$ & SPECT & $\beta^{-}$and $\gamma$ & $\begin{array}{l}\text { Initial staging, response assessment, } \\
\text { therapy \& imaging for dosimetry }\end{array}$ \\
\hline Holmium-166 & $26.8 \mathrm{~h}$ & SPECT & $\beta^{-}$and $\gamma$ & Therapy \& imaging for dosimetry \\
\hline Lutetium-177 & $6.7 \mathrm{~d}$ & SPECT & $\beta^{-}$and $\gamma$ & Therapy \& imaging for dosimetry \\
\hline Rhenium-188 & $17 \mathrm{~h}$ & SPECT & $\beta^{-}$and $\gamma$ & Therapy \& imaging for dosimetry \\
\hline Bismuth-213 & $45.6 \mathrm{~m}$ & - & $\alpha$ & Therapy \\
\hline Radium-223 & $11.4 \mathrm{~d}$ & - & $\alpha$ & Therapy \\
\hline Actinium-225 & $10 \mathrm{~d}$ & - & $\alpha$ & Therapy \\
\hline
\end{tabular}

*Brem - Bremsstrahlung imaging using SPECT

In the areas of dosimetry, treatment planning and monitoring response (figure 2), some other issues today remain the limitations in spatial and temporal resolution of PET and the radiation dose associated with the diagnostic procedure, in particular, the radiation dose from the X-ray CT component of a PET/CT investigation. The latter issue can make studies in normal volunteers challenging whilst remaining within the internationally accepted guidelines for radiation exposure. 


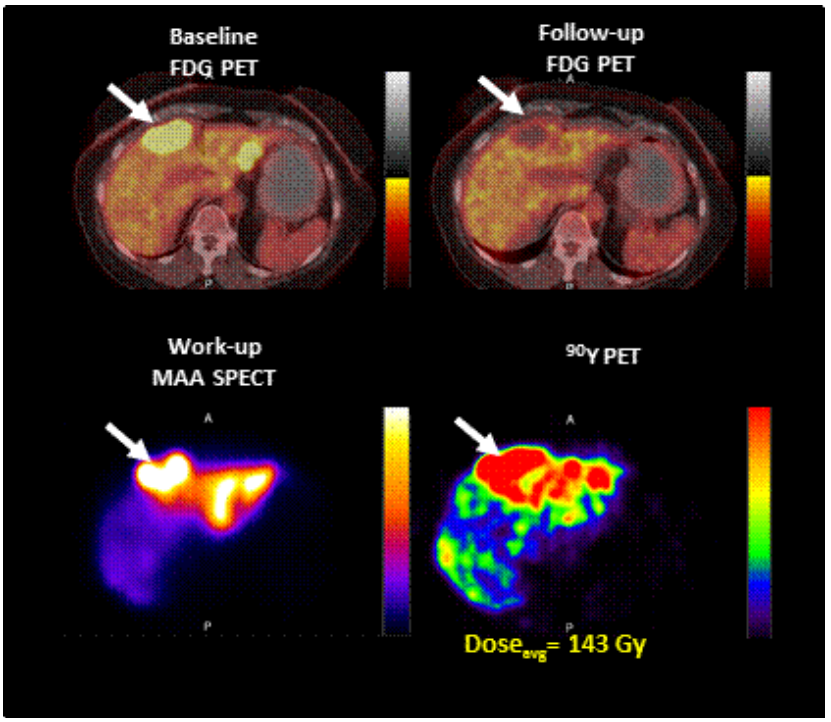

Figure 2. Example of the role of PET imaging in liverdirected treatment for metastatic colorectal carcinoma using $1.8 \mathrm{GBq}$ of $\left[{ }^{90} \mathrm{Y}\right]$-resin microspheres (SIR-Spheres, Sirtex Medical, Australia). The FDG PET scans on the top row show the baseline (left) with increased uptake seen in two predominant lesions and the resolution of the lesions seen 8 weeks after treatment in the follow-up scan (right). The images on the bottom row show the SPECT treatment planning scan (left) using [999mTc]MAA and the distribution of the ${ }^{90} \mathrm{Y}$ microspheres after treatment (right). The ${ }^{90}$ Y PET images are readily converted to dose maps in units of Gy. In this case the predominant lesion was measured to have received 143 Gy averaged over the lesion.

The limited spatial resolution of PET leads to an underestimate of the true concentration of the radiopharmaceutical (or \% Injected Dose per cc) due to the Partial Volume Effect (PVE) in objects under $20 \mathrm{~mm}$ or so in any axis. Table 2 shows the average results for the accuracy of concentration estimation in a PET survey of thirty PET/CT systems from a range of vendors in Australia using the NEMA NU2/IEC Body Phantom containing spheres with a concentration of 8:1 relative to the background. While the single pixel SUV $\mathrm{V}_{\max }$ remained accurate (within $10 \%$ of true value) in objects with a size of just under 3 times the average system spatial resolution (5-6 mm FWHM), the measured concentration for the whole object defined by CT segmentation was underestimated by at least $30 \%$ and was only $50 \%$ in an object diameter of approx. $20 \mathrm{~mm}$. These underestimates would carry through into any dosimetry estimate were mean lesion radiopharmaceutical concentration to be the estimate used. Thus, the PVE remains a major obstacle to PET-based quantification of radiation dose estimates.

Table 2 - Accuracy of maximum and mean radioactivity concentration levels in reconstructed PET images from a site validation exercise surveying $30 \mathrm{PET} / \mathrm{CT}$ scanners in Australia (100\% is the correct value). The values contain data for both ${ }^{18} \mathrm{~F}$ and ${ }^{68} \mathrm{Ga}$. The figure shows a schematic of the phantom used with the locations and sizes (in $\mathrm{mm}$ ) of the spheres contained within plus an example PET image. (Data provided by ARTnet - the Australasian Radiopharmaceutical Trials Network).

\begin{tabular}{|c|c|c|}
\hline $\begin{array}{c}\text { Object Diameter } \\
\text { (mm) }\end{array}$ & $\begin{array}{c}\text { SUV }_{\text {max }} \\
\text { (\%) }\end{array}$ & $\begin{array}{c}\text { SUV }_{\text {mean }} \\
\text { (\%) }\end{array}$ \\
\hline 37 & 102 & 69 \\
\hline 28 & 100 & 61 \\
\hline 22 & 99 & 51 \\
\hline 17 & 93 & 43 \\
\hline 13 & 68 & 28 \\
\hline 10 & 42 & 16 \\
\hline \multicolumn{2}{|c}{} \\
\hline
\end{tabular}
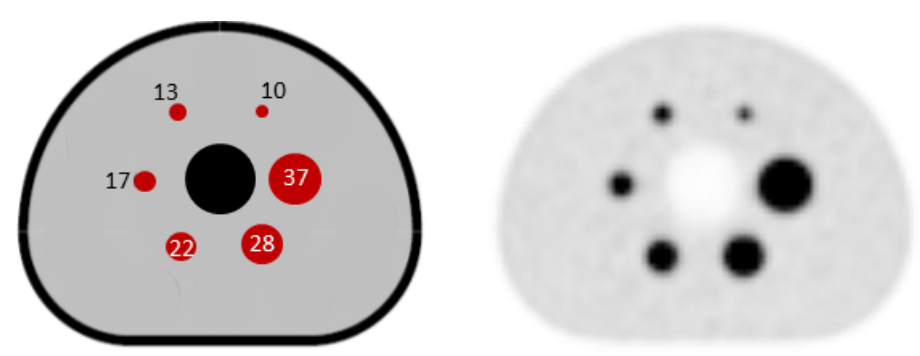

\section{Advances in Science and Technology to Meet Challenges}

Dealing firstly with the radiation dosimetry issue, the recent development of Total Body PET (TB-PET) with vastly increased detection sensitivity will permit high-quality PET imaging at much lower administered radiopharmaceutical levels [1]. This will lead to lower radiation burden to the individuals being imaged. The leaves the CT component as the main contributor to radiation dose in the examination. Here too advances are being made with the adoption in $\mathrm{CT}$ image reconstruction of the 
Ordered Subset Block Iterative methods (OSEM, etc) originally introduced for improving SPECT and PET imaging [143]. Radiation exposure may be reduced by $50 \%$ or more using these reconstruction algorithms. The TB-PET systems also exhibit improved spatial resolution due to improved Time-ofFlight performance of their digital detectors and timing resolution and this will improve the radioactivity concentration estimation accuracy as well.

SPECT remains challenged by extremely limited spatial resolution and, as most of the therapeutic radiopharmaceuticals are $\beta / \gamma$ emitters (i.e., non-PET), improved reconstruction methods will be needed to improve the radiation dosimetry that the gamma camera and SPECT/CT alone can measure.

Finally, the dosimetry that we employ today is very basic and a deeper understanding of the radiobiological effects of radionuclide therapy are required. In particular, the differences between the "slow release" of low dose-rate $\alpha$ or $\beta$ - particles from decaying nuclei for therapy is a vastly different situation to treatment with 3-6 $\mathrm{MV}_{\mathrm{p}} \mathrm{X}$-rays at $\mathrm{cGy} / \mathrm{sec}$ from a modern linear accelerator used in radiotherapy. Early comparisons show up to a 10-fold difference in certain biological parameters for cell survival with the high-energy X-rays being far more damaging to cells [144].

\section{Concluding Remarks}

Nuclear Medicine functional imaging is enjoying a dramatic increase in acceptance into clinical medicine and is providing demonstrable, transformational changes in outcomes for patients, especially those with certain cancers. The boundaries between SPECT and PET, diagnosis and therapy, and imaging assessments that are combined with other modalities are becoming blurred. The role of quantitative PET in treatment planning and dosimetry will likely increase using newer radionuclides with longer half-lives. The greatest challenge today with the tools at hand is to generate the evidential base on which to guide future diagnostic pathways and tailored treatment for each individual. 


\section{The role of $A l$ in quantitative PET Joyita Dutta \\ Department of Electrical and Computer Engineering, University of Massachusetts Lowell}

\section{Status}

Recent advances in machine learning and artificial intelligence (Al), particularly the emergence of innovative deep neural network architectures, have been transformative for medical imaging. Specifically, for PET imaging, where accurate quantitative interpretation has been both a pressing need and a lingering challenge, Al has assumed a growing role in improving image quantitation and facilitating image interpretation. The former category includes image reconstruction and image processing/restoration techniques, while the latter spans target detection and radiomics as illustrated in Figure 1. The two key confounding factors that compromise PET image quality and quantitation are spatial resolution and noise. Consequently, deblurring and denoising have been the primary motivations for developing Al-based approaches for PET image processing and reconstruction. Albased PET image reconstruction techniques fall under three categories: (1) data-driven, end-to-end approaches that perform a domain transform receiving raw data (sinogram) as the input and generating images as the output [145], (2) model-driven techniques that unroll the iterative reconstruction process flow by using the network layers as individual iterations for image updates [146], and (3) penalty-based approaches which preserve the model-based iterative reconstruction format (and hence exploit the strengths of known physical and statistical models) while incorporating population-level learned information into a deep-learning-based image model (i.e., a prior or penalty function) [147]. Al-based solutions for accurate attenuation correction (in the case of PET/MR) and efficient scatter estimation have also facilitated PET image reconstruction [148]. Besides image reconstruction, deep neural networks that leverage anatomical information have been successfully used to restore PET images through image-domain processing. These methods include denoising strategies for low-count PET images with poor signal-to-noise ratio [149] and super-resolution strategies for PET images with poor spatial resolution [150]. Neural networks are also increasingly being employed to facilitate accurate interpretation of PET images. These applications include detection/classification problems, e.g., automated detection of lesions in PET/CT [151] and lesion segmentation. Deep learning techniques have also permeated the broader field of radiomics, which involves high-throughput extraction of quantitative metrics from medical images and enabled automatic feature discovery obviating the need for an intermediate lesion segmentation step.

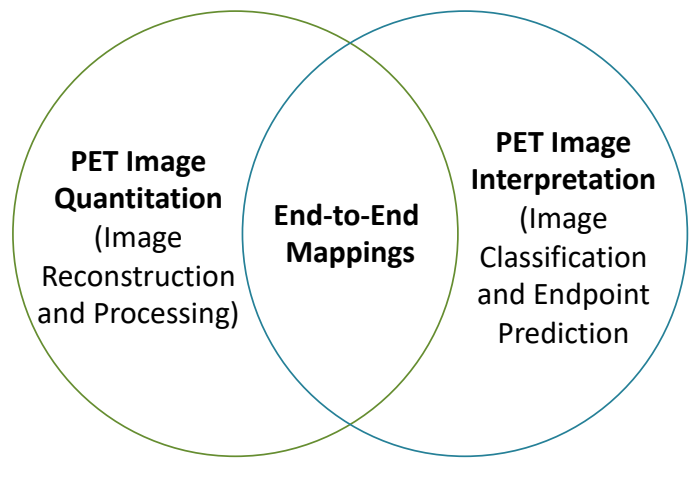

Figure 2: Quantitative interpretation of PET images 


\section{Current and Future Challenges}

Despite the success and rapid expansion of deep learning in applications surrounding quantitative PET imaging, some key challenges restricting the wider adoption of many of these approaches are:

(1) Data Size Limitations: The first challenge centres around the need for PET image repositories with data volume and variety to impart robustness to deep learning models. Many of the approaches described previously rely on popular convolutional architectures (e.g., the U-Net) trained in supervised mode, which means the training process requires paired input-output combinations. The very high accuracy of supervised learning techniques, however, is contingent on the datasets for final use being very similar to those used for training often limiting their wider use. In the context of PET imaging, this not only implies the need for data based on the same tracer type but also data with similar tracer dose, scanner geometry, scan duration, etc. Unlike CT or MR scans, PET images have spatially non-uniform resolution and noise characteristics making the training phase design challenging. Even with a fixed tracer injection dose, the uptake and biodistribution can greatly vary across individuals and lead to a high degree of variation in the noise level.

(2) Model Interpretability: The second challenge is posed by the "black box" nature of neural networks. While, in general, representation learning paradigms are capable of end-to-end learning and could, at least in principle, be used to predict clinical outcome measures directly from raw patient data, the relative complexity of these models and the inability to generate simple explanations of their process flow often impedes clinical translation and adoption as decision-making tools.

\section{Advances in Science and Technology to Meet Challenges}

Advances in the science and technology needed to meet the challenges of quantitative PET fall into two broad categories for which the current pace of innovation in today's Al landscape is among the fastest:

(1) Advances in Unsupervised Learning Techniques for Image Estimation and Classification: Recognizing the need for learning techniques that can utilize unlabelled datasets, lately there has been a surge in unsupervised, semi-supervised, and self-supervised learning techniques in all domains of Al research. In the context of image estimation, there is a need for learning techniques that do not rely on paired training image sets. Sophisticated generative adversarial network (GAN) architectures, such as the cycle-consistent GAN (CycleGAN) and variants, have led to innovative solutions to the PET image denoising [152] and super-resolution [150] problems. Fig. 2 shows a comparison of denoising performance of UNet, GAN, and CycleGAN. The availability of such new learning approaches and network designs that allow input and output (target) images used for network training to be derived from different image pools will impart flexibility and access to larger data pools from distinct cohorts to be used for training. Advances in image classification techniques that obviate the need for manual labelling of training images will also lead to robust, high-throughput, automated lesion detection.

(2) Advances in Explainable Techniques for Image Interpretation: Quantitative PET imaging currently plays a vital role in diagnostics, staging, and therapeutic evaluation for diseases with applications spanning oncology, neurology, cardiology, and other fields. With the increasingly prominent role of $\mathrm{Al}$ in clinical decision support systems, end-to-end mapping techniques based on PET datasets are expected to be on the rise. Higher levels of transparency are required in these cases to explain machine decisions. Techniques such as saliency maps and attention mechanisms have led to improved model understanding in various Al problems. 
Future advances in these areas will catalyse the clinical translation of end-to-end learning systems that use PET datasets as inputs either standalone or with information from imaging and non-imaging data sources.
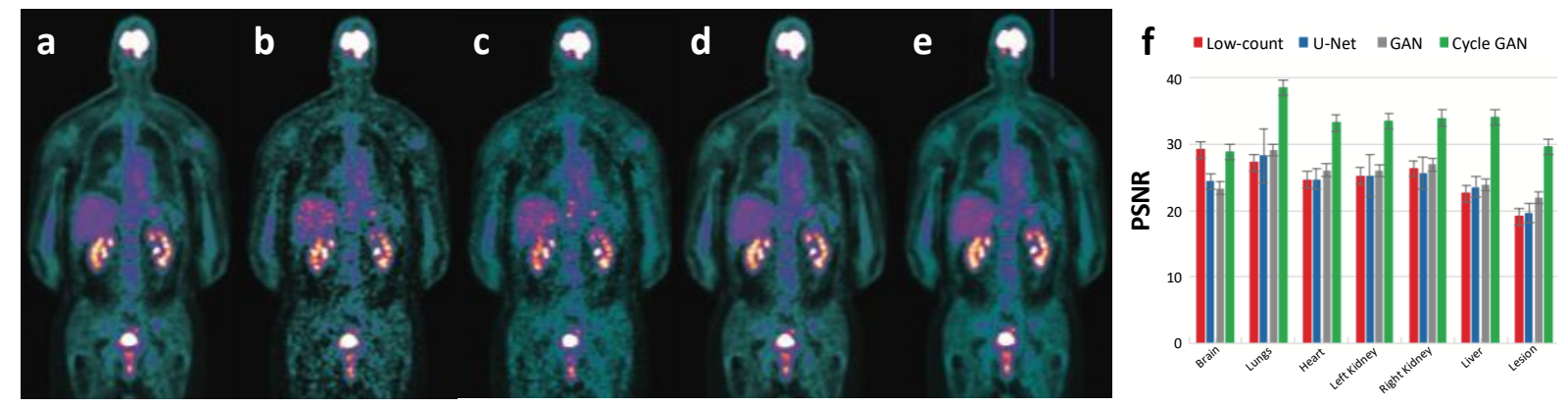

Figure 3: Comparison of denoising performance of different neural networks: (a) Full-count (b) low-count, (c) U-Net denoised, (d) GAN denoised, and (e) CycleGAN denoised images representing coronal slices from a whole-body clinical PET scan. (f) Peak signal-to-noise ratio (PSNR) comparison for the noisy and denoised images using the full-count image as the ground truth. (Adapted from [153]).

\section{Concluding Remarks}

Al has already had a profound influence on all domains of quantitative PET, including estimation and classification problems, where traditional physics and statistics-based modelling fall short. These complex models have successfully tackled the uncertainties in image processing posed by the spatially variant and iteration-dependent noise and resolution characteristics of PET images that traditional approaches have struggled with. They have offered new avenues for incorporating anatomical information to improve PET quantitation. Finally, they have enabled end-to-end image-based prediction of clinical endpoints. While initially developed for static PET images, these methods are beginning to be adopted for dynamic PET datasets at a fast pace. Clinical translation of Al approaches, which are usually validated using image-based metrics such as bias, variance, peak signal-to-noise ratio, etc., will require increased emphasis on task-based validation. In the upcoming years, the integration of Al-based image quality improvements and Al-based clinical decision making will go hand in hand toward revolutionizing precision medicine.

\section{Acknowledgements}

This work was supported in part by the NIH grant K01AG050711. 


\section{Epilogue}

Steven R Meikle1, Terry Jones ${ }^{2}$, Simon R Cherry ${ }^{3,2}$,

1 Sydney School of Health Sciences, The University of Sydney

2 Department of Radiology, University of California, Davis

3 Department of Biomedical Engineering, University of California, Davis

Positron Emission Tomography (PET) is the most specific, sensitive, and quantitative means for undertaking molecular imaging in humans. It will be seen from the contributions to this Roadmap, that the underlying technologies and methodologies that form the overall PET data are destined to increase the sensitivity and specificity of PET derived molecular images. While this will reinforce those clinical disciplines currently using PET, for example in the brain and oncology, it will also provide opportunities to widen the use of PET outside of those areas. An example of this is molecular imaging of the human body as a system. This would enable the means to study interactions between the brain and the rest of the body as well as the kinetics of a drug's distribution and its functional response across the whole body. A more poignant example is that it offers the means to help research the impact of the body's functional reactions to infections such as the COVID-19 virus. This devastating disease is presenting as a complex, highly varied pathophysiology, arising from the by no means fully understood malfunctioning of the body's immune response.

To reinforce such future developments, it is important that there is in place a corresponding path to ensure the emerging data can be presented in a quantitative form. The scientific method rests on reporting the results of investigations in a quantitative manner that can be comprehended by the scientific community. The quantitative format fosters those clinical scientists, unfamiliar with the intricacies of the underlying methodology of PET, to ask clinical scientific questions using this unique investigative tool. On translating the developed PET methodology to healthcare, reporting of functional imaging data in a quantitative form is by far the most impacting and robust means for such communication.

This Roadmap is, by definition, forward looking. We invited leading researchers in all the subdisciplines of PET to offer a glimpse into the unknown future, drawing on their personal perspectives on the current state of the field and their experience. If only half of their predictions become reality, the decade ahead will be very exciting. Indeed, there likely are future realities none of us can anticipate that will be even more impactful. It is clear that we are on the cusp of a significant expansion in the applications space. However, if the field is to fully exploit these new opportunities, it will need to rediscover its quantitative DNA and address a host of new challenges.

Many of these new and expanded applications involve imaging and quantifying sparse targets with potentially high levels of non-specific background, such as receptors in the spine and gut, circulating macrophages or activated T cells. To meet these exacting demands, new radiotracers with high specificity and molar activity will be required. Also, low statistics datasets will present themselves as we aim to reduce the levels of administered radioactivity to minimise radiation absorbed doses to the more radiation sensitive populations. We also need to reduce the time it takes to identify the most promising candidate imaging biomarkers and bring them more quickly to practical utilisation in research laboratories and clinics. 
Despite impressive gains in sensitivity arising from the introduction of Total Body PET and the opportunities arising from PET/MRI, emerging applications will continue to push PET instrumentation towards new limits of performance. There remains considerable room to further improve the time-offlight resolution of PET scanners through faster detectors and electronics and, as a consequence, we can expect effective sensitivity of these systems to continue increasing over the coming decade. Likewise, the spatial resolution of whole body tomographs is still some way short of the theoretical limits for the most common positron emitters and there are almost unlimited opportunities to make further gains through the design of dedicated, application-specific PET systems, in particular for the brain. We also need to continue exploring novel ways to correct for motion in all organs of the body, scatter, randoms and partial volume effects, and more fully exploit the synergies between PET and MRI.

A relatively safe prediction is that the new decade will be the decade of big data, arising not only from the greatly enhanced sensitivity of PET scanners, but also from complementary imaging modalities, omics platforms and other relevant clinical outcome measures. The challenges include: how to optimise the use of information in this multidimensional data space, how to reliably and reproducibly estimate biologically relevant parameters at the voxel level and their uncertainties, and how best to exploit the power of artificial intelligence to help us achieve these goals. It is also important to consider how we standardise and harmonise protocols so that our results can be replicated and how we make our technology more globally accessible. Considering that many of the potential new applications involve imaging multiple organs and tissues simultaneously, there will be specific challenges for image reconstruction and tracer kinetic modelling, such as robust direct quantitative estimation of kinetic parameters in multiple organs and tissues, each with potentially different (or multiple) input functions, kinetic compartmentalization, heterogeneity, and metabolite profiles. Similarly, more sophisticated modelling of micro-dosimetry and radiobiological effects is needed to harness the opportunities arising from the expanding range of theranostic agents, thus enabling more effective, personalised treatment planning.

Finally, several contributors highlighted the need to direct more resources into education and training of the next generation of PET scientists. The need is particularly acute in radiochemistry and tracer kinetic modelling. These two highly complementary areas of specialisation in our field continue to be the major bottlenecks in bringing new, well-characterised radiotracers to the clinic which are amenable to robust, reproducible, and efficient estimation of biologically important outcome measures. We also need to place more emphasis on communicating the opportunities PET provides to our colleagues in cognate disciplines, especially those who have not previously used it in their research or clinical disciplines and on lowering the cost of PET technology to increase accessibility while addressing the needs of developing countries and remote communities.

The future of quantitative PET is extremely bright. There are rich seams of opportunity to be tapped and a vast catalogue of challenging problems to solve. Biomedical physicists will play an essential part, but collaboration across disciplines will be key to the success and ultimate impact on human health of the directions outlined in this roadmap. 


\section{References}

[1] Badawi R D, Shi H, Hu P, Chen S, Xu T, Price P M, Ding Y, Spencer B A, Nardo L, Liu W, Bao J, Jones T, Li H and Cherry S R 2019 First human imaging studies with the EXPLORER total-body PET scanner J. Nucl. Med. 60 299-303

[2] Pantel A R, Viswanath V, Daube-Witherspoon M E, Dubroff J G, Muehllehner G, Parma M J, Pryma D A, Schubert E K, Mankoff D A and Karp J S 2020 PennPET Explorer: Human imaging on a whole-body imager J. Nucl. Med. 61 144-51

[3] Gallezot J-D, Lu Y, Naganawa M and Carson R E 2019 Parametric Imaging with PET and SPECT IEEE Trans. Radiat. Plasma Med. Sci. 4 1-23

[4] Houser M C and Tansey M G 2017 The gut-brain axis: is intestinal inflammation a silent driver of Parkinson's disease pathogenesis? npj Park. Dis. 3

[5] Kitai T and Tang W H W 2018 Gut microbiota in cardiovascular disease and heart failure Clin. Sci. 132 85-91

[6] Li T, Ao E C I, Lambert B, Brans B, Vandenberghe S and Greta S P 2017 Quantitative imaging for targeted radionuclide therapy dosimetry - technical review Theranostics 7 4551-65

[7] Boellaard R 2009 Standards for PET Image Acquisition and Quantitative Data Analysis J Nucl Med 50 11-20

[8] Fahey F, Kinahan P, Doot R, Kocak M, Thurston H and Poussaint T 2010 Variability in PET quantitation within a multicenter consortium. Med Phys 37 3660-6

[9] Gundacker S, Turtos R M, Kratochwil N and Pots R H 2020 Experimental time resolution limits of modern SiPMs and TOF-PET detectors exploring different scintillators and Cherenkov emission Experimental time resolution limits of modern SiPMs and TOF-PET detectors exploring different scintillators and Cherenkov em Phys Med Biol 65025001

[10] Visvikis D, Cheze Le Rest C, Jaouen V and Hatt M 2019 Artificial intelligence, machine (deep) learning and radio(geno)mics: definitions and nuclear medicine imaging applications Eur. J. Nucl. Med. Mol. Imaging 46 2630-7

[11] Jones T and Rabiner E A 2012 The development, past achievements, and future directions of brain PET J. Cereb. Blood Flow Metab. 32 1426-54

[12] Henstridge C M, Tzioras M and Paolicelli R C 2019 Glial contribution to excitatory and inhibitory synapse loss in neurodegeneration Front. Cell. Neurosci. 13 1-26

[13] Banati R B 2003 Neuropathological imaging: in vivo detection of glial activation as a measure of disease and adaptive change in the brain Br. Med. Bull. 65 121-31

[14] Liu G J, Middleton R J, Hatty C R, Kam W W Y, Chan R, Pham T, Harrison-Brown M, Dodson E, Veale K and Banati R B 2014 The 18 kDa translocator protein, microglia and neuroinflammation Brain Pathol. 24 631-53

[15] Banati R B, Middleton R J, Chan R, Hatty C R, Wai-Ying Kam W, Quin C, Graeber M B, Parmar A, Zahra D, Callaghan P, Fok S, Howell N R, Gregoire M, Szabo A, Pham T, Davis E and Liu G J 2014 Positron emission tomography and functional characterization of a complete PBR/TSPO knockout Nat. Commun. 5 1-12

[16] Villemagne V L, Doré V, Burnham S C, Masters C L and Rowe C C 2018 Imaging tau and amyloid$\beta$ proteinopathies in Alzheimer disease and other conditions Nat. Rev. Neurol. 14 225-36

[17] Morris G P, Clark I A and Vissel B 2018 Questions concerning the role of amyloid- $\beta$ in the definition, aetiology and diagnosis of Alzheimer's disease Acta Neuropathol. 136 663-89

[18] Leuzy A, Chiotis K, Lemoine L, Gillberg P G, Almkvist O, Rodriguez-Vieitez E and Nordberg A 2019 Tau PET imaging in neurodegenerative tauopathies-still a challenge Mol. Psychiatry 24 1112-34 
[19] Kas A, Migliaccio R and Tavitian B 2020 A future for PET imaging in Alzheimer's disease Eur. J. Nucl. Med. Mol. Imaging 47 231-4

[20] Luurtsema G, Elsinga P, Dierckx R, Boellaard R and van Waarde A 2016 PET Tracers for Imaging of ABC Transporters at the Blood-Brain Barrier: Principles and Strategies Curr Pharm Des. 22 5779-85

[21] Kiernan M C, Vucic S, Cheah B C, Turner M R, Eisen A, Hardiman O, Burrell J R and Zoing M C 2011 Amyotrophic lateral sclerosis Lancet 377 942-55

[22] Freund P, Friston K, Thompson A J, Stephan K E, Ashburner J, Bach D R, Nagy Z, Helms G, Draganski B, Mohammadi S, Schwab M E, Curt A and Weiskopf N 2016 Embodied neurology: An integrative framework for neurological disorders Brain 139 1855-61

[23] Stroman P W, Wheeler-Kingshott C, Bacon M, Schwab J M, Bosma R, Brooks J, Cadotte D, Carlstedt T, Ciccarelli O, Cohen-Adad J, Curt A, Evangelou N, Fehlings M G, Filippi M, Kelley B J, Kollias S, Mackay A, Porro C A, Smith S, Strittmatter S M, Summers P and Tracey I 2014 The current state-of-the-art of spinal cord imaging: Methods Neuroimage 84 1070-81

[24] Song T A, Chowdhury S R, Yang F and Dutta J 2020 PET image super-resolution using generative adversarial networks Neural Networks 125 83-91

[25] Friedman A A, Letai A, Fisher D E and Flaherty K T 2015 Precision medicine for cancer with next-generation functional diagnostics Nat. Rev. Cancer 15 747-56

[26] Mankoff D A, Farwell M D, Clark A S and Pryma D A 2017 Making molecular imaging a clinical tool for precision oncology: A review JAMA Oncol. 3 695-701

[27] Kinahan P E, Perlman E S, Sunderland J J, Subramaniam R, Wollenweber S D, Turkington T G, Lodge M A, Boellaard R, Obuchowski N A and Wahl R L 2020 The QIBA profile for FDG PET/CT as an imaging biomarker measuring response to cancer therapy Radiology 294 647-57

[28] Loke K S H, Padhy A K, Ng D C E, Goh A S W and Divgi C 2011 Dosimetric Considerations in Radioimmunotherapy and Systemic Radionuclide Therapies: A Review World J. Nucl. Med. 10 $122-38$

[29] Peterson L M, O'Sullivan J, Wu Q, Novakova-Jiresova A, Jenkins I, Lee J H, Shields A, Montgomery S, Linden H M, Gralow J, Gadi V K, Muzi M, Kinahan P, Mankoff D and Specht J M 2018 Prospective study of serial18F-FDG PET and18F-fluoride PET to predict time to skeletalrelated events, time to progression, and survival in patients with bone-dominant metastatic breast cancer J. Nucl. Med. 59 1823-30

[30] Wahl R L, Jacene H, Kasamon $Y$ and Lodge M A 2009 From RECIST to PERCIST: Evolving considerations for PET response criteria in solid tumors J. Nucl. Med. $\mathbf{5 0}$

[31] Dunnwald L K, Doot R K, Specht J M, Gralow J R, Ellis G K, Livingston R B, Linden H M, Gadi V K, Kurland B F, Schubert E K, Muzi M and Mankoff D A 2011 PET tumor metabolism in locally advanced breast cancer patients undergoing neoadjuvant chemotherapy: Value of static versus kinetic measures of fluorodeoxyglucose uptake Clin. Cancer Res. 17 2400-9

[32] Linden H M, Stekhova S A, Link J M, Gralow J R, Livingston R B, Ellis G K, Petra P H, Peterson L M, Schubert E K, Dunnwald L K, Krohn K A and Mankoff D A 2006 Quantitative fluoroestradiol positron emission tomography imaging predicts response to endocrine treatment in breast cancer J. Clin. Oncol. 24 2793-9

[33] Kuker R, Sztejnberg M and Gulec S 2017 I-124 Imaging and dosimetry Mol. Imaging Radionucl. Ther. 26 66-73

[34] Linden H, Stekhova S, Link J, Gralow J, Livingston R, Ellis G, Petra P, Peterson L, Schubert E, Dunnwald L, Krohn K and Mankoff D 2006 Quantitative fluoroestradiol positron emission tomography imaging predicts response to endocrine treatment in breast cancer J Clin Oncol 24 
2793-9

[35] Cherry S R, Badawi R D, Karp J S, Moses W W, Price P and Jones T 2017 Total-body imaging: Transforming the role of positron emission tomography Sci. Transl. Med. 1-4

[36] El Naqa I, Pandey G, Aerts H, Chien J T, Andreassen C N, Niemierko A and Ten Haken R K 2018 Radiation Therapy Outcomes Models in the Era of Radiomics and Radiogenomics: Uncertainties and Validation Int. J. Radiat. Oncol. Biol. Phys. 102 1070-3

[37] Jones T and Townsend D 2017 History and future technical innovation in positron emission tomography J. Med. Imaging 4011013

[38] Mellman I, Coukos G and Dranoff G 2011 Cancer immunotherapy comes of age Nature 480 480-9

[39] Friston K J, Holmes A P, Worsley K J, Poline J-P, Frith C D and Frackowiak R S J 1994 Statistical parametric maps in functional imaging: A general linear approach Hum. Brain Mapp. 2 189210

[40] Cherry S R, Jones T, Karp J S, Qi J, Moses W and Badawi R D 2017 Total-Body PET: Maximizing Sensitivity To Create New Opportunities for Clinical Research and Patient Care J. Nucl. Med. jnumed.116.184028

[41] Catana C 2019 Development of dedicated brain PET imaging devices: Recent advances and future perspectives J. Nucl. Med. 60 1044-52

[42] Lecoq P 2017 Pushing the Limits in Time-of-Flight PET Imaging IEEE Trans. Radiat. Plasma Med. Sci. $1473-85$

[43] Zhang X, Xie Z, Berg E, Judenhofer M S, Liu W, Xu T, Ding Y, Lv Y, Dong Y, Deng Z, Tang S, Shi H, Hu P, Chen S, Bao J, Li H, Zhou J, Wang G, Cherry S R, Badawi R D and Qi J 2019 Total-Body Dynamic Reconstruction and Parametric Imaging on the UEXPLORER J. Nucl. Med. jnumed.119.230565

[44] Wang G and Qi J 2015 PET image reconstruction using kernel method IEEE Trans. Med. Imaging 34 61-71

[45] Ashburner J, Haslam J, Taylor C, Cunningham V and Jones T 1996 A cluster analysis approach for the characterization of dynamic PET data

[46] Walker S M, Lim I, Lindenberg L, Mena E, Choyke P L and Turkbey B 2020 Positron emission tomography (PET) radiotracers for prostate cancer imaging Abdom. Radiol.

[47] Pollard J, McNeely P and Menda Y 2020 Nuclear Imaging of Neuroendocrine Tumors Surg. Oncol. Clin. N. Am.

[48] Uzuegbunam B C, Librizzi D and Yousefi B H 2020 PET radiopharmaceuticals for Alzheimer's disease and Parkinson's disease diagnosis, the current and future landscape Molecules

[49] Solnes L B, Werner R A, Jones K M, Sadaghiani M S, Bailey C R, Lapa C, Pomper M G and Rowe S P 2020 Theranostics: Leveraging Molecular Imaging and Therapy to Impact Patient Management and Secure the Future of Nuclear Medicine J. Nucl. Med.

[50] Mattsson N, Carrillo M C, Dean R A, Devous M D, Nikolcheva T, Pesini P, Salter H, Potter W Z, Sperling R S, Bateman R J, Bain L J and Liu E 2015 Revolutionizing Alzheimer's disease and clinical trials through biomarkers Alzheimer's Dement. Diagnosis, Assess. Dis. Monit.

[51] Gagnon M K J, Hausner S H, Marik J, Abbey C K, Marshall J F and Sutcliffe J L 2009 Highthroughput in vivo screening of targeted molecular imaging agents Proc. Natl. Acad. Sci. U.S. A. 106 17904-9

[52] Davis R A, Hausner S H and Sutcliffe J L 2019 Peptides as Radiopharmaceutical Vectors Radiopharmaceutical Chemistry pp 137-62 
[53] Cherry S R, Jones T, Karp J S, Qi J, Moses W and Badawi R 2017 Total-Body Pet: Maximizing Sensitivity To Create New Opportunities for Clinical Research and Patient Care J. Nucl. Med. jnumed.116.184028

[54] Cipriano P W, Yoon D, Gandhi H, Holley D, Thakur D, Hargreaves B A, Kennedy D J, Smuck M W, Cheng I and Biswal S 201818 F-FDG PET/MRI in chronic sciatica: Early results revealing spinal and nonspinal abnormalities J. Nucl. Med.

[55] Grapov D, Fahrmann J, Wanichthanarak K and Khoomrung S 2018 Rise of deep learning for genomic, proteomic, and metabolomic data integration in precision medicine Omi. A J. Integr. Biol.

[56] Carpenter K A, Cohen D S, Jarrell J T and Huang X 2018 Deep learning and virtual drug screening Future Med. Chem.

[57] Shao Y, Cherry S R, Farahani K, Meadors K, Siegal S, Silverman R W and Marsden P K 1997 Simultaneous PET and MR imaging Phys. Med. Biol. 42 1965-70

[58] Bai B, Li Q and Leahy R M 2013 Magnetic resonance-guided positron emission tomography image reconstruction Semin. Nucl. Med. 43 30-44

[59] Mehranian A, Belzunce M A, Prieto C, Hammers A and Reader A J 2018 Synergistic PET and SENSE MR Image Reconstruction Using Joint Sparsity Regularization IEEE Trans. Med. Imaging 37 20-34

[60] Mandeville J B, Sander C Y M, Jenkins B G, Hooker J M, Catana C, Vanduffel W, Alpert N M, Rosen B R and Normandin M D 2013 A receptor-based model for dopamine-induced fMRI signal Neuroimage 75 46-57

[61] Alpert N M, Guehl N, Ptaszek L, Pelletier-Galarneau M, Ruskin J, Mansour M C, Wooten D, Ma C, Takahashi K, Zhou Y, Shoup T M, Normandin M D and Fakhri G El 2018 Quantitative in vivo mapping of myocardial mitochondrial membrane potential PLoS One 13 e0190968

[62] Cabello J, Lukas M, Förster S, Pyka T, Nekolla S G and Ziegler S I 2015 MR-based attenuation correction using ultrashort-echo-time pulse sequences in dementia patients J. Nucl. Med. 56 423-9

[63] Sekine T, Buck A, Delso G, Ter Voert E E G W, Huellner M, Veit-Haibach P and Warnock G 2016 Evaluation of atlas-based attenuation correction for integrated PET/MR in human brain: Application of a head atlas and comparison to true ct-based attenuation correction J. Nucl. Med. 57 215-20

[64] Leynes A P, Yang J, Wiesinger F, Kaushik S S, Shanbhag D D, Seo Y, Hope T A and Larson P E Z 2018 Zero-echo-time and dixon deep pseudo-CT (ZeDD CT): Direct generation of pseudo-CT images for Pelvic PET/MRI Attenuation Correction Using Deep Convolutional Neural Networks with Multiparametric MRI J. Nucl. Med. 59 852-8

[65] Huang C, Ackerman J L, Petibon Y, Normandin M D, Brady T J, El Fakhri G and Ouyang J 2014 Motion compensation for brain PET imaging using wireless MR active markers in simultaneous PET-MR: Phantom and non-human primate studies Neuroimage 91 129-37

[66] Petibon Y, Sun T, Han P K, Ma C, Fakhri G El and Ouyang J 2019 MR-based cardiac and respiratory motion correction of PET: Application to static and dynamic cardiac 18F-FDG imaging Phys. Med. Biol. 64

[67] Kennedy J A, Israel O, Frenkel A, Bar-Shalom R and Haim Azhari 2006 Super-resolution in PET imaging IEEE Trans. Med. Imaging 25 137-47

[68] Yuan H, Wilks M Q, El Fakhri G, Normandin M D, Kaittanis C and Josephson L 2017 Heatinduced-radiolabeling and click chemistry: A powerful combination for generating multifunctional nanomaterials PLoS One 12 e0172722 
[69] Lewellen T K 1998 Time-of-flight PET Semin. Nucl. Med. 28 268-75

[70] Surti S and Karp J S 2016 Advances in time-of-flight PET Phys. Medica 32 12-22

[71] Pan T, Einstein S A, Kappadath S C, Grogg K S, Lois Gomez C, Alessio A M, Hunter W C, El Fakhri G, Kinahan P E and Mawlawi O R 2019 Performance evaluation of the 5-Ring GE Discovery MI $\mathrm{PET} / \mathrm{CT}$ system using the national electrical manufacturers association NU 2-2012 Standard Med. Phys. 46 3025-33

[72] Van Sluis J, De Jong J, Schaar J, Noordzij W, Van Snick P, Dierckx R, Borra R, Willemsen A and Boellaard R 2019 Performance characteristics of the digital biograph vision PET/CT system J. Nucl. Med. 60 1031-6

[73] Rausch I, Ruiz A, Valverde-Pascual I, Cal-González J, Beyer T and Carrio I 2019 Performance evaluation of the VereoS PET/CT system according to the NEMA NU2-2012 standard J. Nucl. Med. 60 561-7

[74] Surti S 2015 Update on time-of-flight PET imaging J. Nucl. Med. 56 98-105

[75] Cates J W and Levin C S 2016 Advances in coincidence time resolution for PET. Phys. Med. Biol. $612255-64$

[76] Frach T, Degenhardt C, Jülich F, Prescher G, De Gruyter R, Schmitz A and Ballizany R 2009 The Digital Silicon Photomultiplier - Principle of Operation and Intrinsic Detector Performance

[77] Borghi G, Tabacchini V and Schaart D R 2016 A $32 \mathrm{~mm} \times 32 \mathrm{~mm} \times 22 \mathrm{~mm}$ monolithic LYSO:Ce detector with dual-sided digital photon counter readout for ultrahigh-performance TOF-PET and TOF-PET/MRI Phys. Med. Biol 61 4929-4949

[78] Cates J W, Gundacker S, Auffray E, Lecoq P and Levin C S 2018 Improved single photon time resolution for analog SiPMs with front end readout that reduces influence of electronic noise. Phys. Med. Biol. 63185022

[79] Lecoq P, Morel C, Prior J, Visvikis D, Gundacker S, Auffray E, Krizan P, Turtos R M, Thers D, Charbon E, Varela J, de La Taille C, Rivetti A, Breton D, Pratte J-F, Nuyts J, Surti S, Vandenberghe S, Marsden P K, Parodi K, Benlloch J M and Benoit M 2020 Roadmap toward the 10 ps time-offlight PET challenge Phys. Med. Biol. in press (accepted 20 May 2020)

[80] Kay S M 1993 Fundamentals of statistical signal processing, Volume 1: Estimation theory (Prentice Hall)

[81] Gundacker S, Auffray E, Pauwels K and Lecoq P 2016 Measurement of intrinsic rise times for various $L(Y) S O$ and LuAG scintillators with a general study of prompt photons to achieve 10 ps in TOF-PET Phys Med Biol 61 2802-37

[82] Poon J K, Dahlbom M L, Moses W W, Balakrishnan K, Wang W, Cherry S R and Badawi R D 2012 Optimal whole-body PET scanner configurations for different volumes of LSO scintillator: a simulation study. Phys. Med. Biol. 57 4077-94

[83] Zhang X, Cherry S R, Xie Z, Shi H, Badawi R D and Qi J 2020 Subsecond total-body imaging using ultrasensitive positron emission tomography Proc. Natl. Acad. Sci. 201917379

[84] Viergever M A, Maintz J B A, Klein S, Murphy K, Staring M and Pluim J P W 2016 A survey of medical image registration - under review Med. Image Anal. 33 140-4

[85] Merlin T, Visvikis D, Fernandez P and Lamare F 2018 Dynamic PET image reconstruction integrating temporal regularization associated with respiratory motion correction for applications in oncology Phys Med Biol 63045012

[86] Gong K, Berg E, Cherry S R and Qi J 2020 Machine Learning in PET: From Photon Detection to Quantitative Image Reconstruction Proc. IEEE 108 51-68

[87] Rothfuss H, Panin V, Moor A, Young J, Hong I, Michel C, Hamill J and Casey M 2014 LSO background radiation as a transmission source using time of flight Phys. Med. Biol. 59 5483- 
500

[88] Kuntner C and Stout D 2014 Quantitative preclinical PET imaging: Opportunities and challenges Front. Phys. 2 1-12

[89] Gonzalez A J, Sanchez F and Benlloch J M 2018 Organ-Dedicated Molecular Imaging Systems IEEE Trans. Radiat. Plasma Med. Sci. 2 388-403

[90] Parodi K and Polf J C 2018 In vivo range verification in particle therapy Med. Phys. 45 e103650

[91] Liang J, Da Silva A, Han C, Neylon J, Amini A, Sampath S, Liu A and Wong J 2019 Biology-guided Radiotherapy for Lung SBRT Reduces Planning Target Volumes and Organs at Risks Doses Int. J. Radiat. Oncol. Biol. Phys. 105254

[92] Liyanaarachchi M R, Shimazoe K, Takahashi H, Kobayashi E, Nakagawa K and Sakuma I 2020 Prototype detector for intraoperative PET-laparoscope system with a multi-layer movable detector Nucl. Instruments Methods Phys. Res. Sect. A Accel. Spectrometers, Detect. Assoc. Equip. 958162788

[93] Aubry N, Auffray E, Mimoun F B, Brillouet N, Bugalho R, Charbon E, Charles O, Cortinovis D, Courday P, Cserkaszky A, Damon C, Doroud K, -M Fischer J, Fornaro G, Fourmigue J M, Frisch B, Fürst B, Gardiazabal J, Gadow K, Garutti E, Gaston C, Gil-Ortiz A, Guedj E, Harion T, Jarron P, Kabadanian J, Lasser T, Laugier R, Lecoq P, Lombardo D, Mandai S, Mas E, Meyer T, Mundler O, Navab N, Ortigão C, Paganoni M, Perrodin D, Pizzichemi M, Prior J O, Reichl T, Reinecke M, Rolo M, -C Schultz-Coulon H, Schwaiger M, Shen W, Silenzi A, Silva J C, Silva R, Somlai Schweiger I, Stamen R, Traub J, Varela J, Veckalns V, Vidal V, Vishwas J, Wendler T, Xu C, Ziegler S and Zvolsky M 2013 EndoTOFPET-US: A novel multimodal tool for endoscopy and positron emission tomography J. Instrum. 8 C04002

[94] Mohammadi I, Castro I F C, Correia P M M, Silva A L M and Veloso J F C A 2019 Minimization of parallax error in positron emission tomography using depth of interaction capable detectors: methods and apparatus Biomed. Phys. Eng. Express 5062001

[95] Vandenberghe S, Mikhaylova E, D'Hoe E, Mollet P and Karp J S 2016 Recent developments in time-of-flight PET EJNMMI Phys. 3 1-30

[96] Nakano T, Sakai M, Torikai K, Suzuki Y, Takeda S, Noda S, Yamaguchi M, Nagao Y, Kikuchi M, Odaka H, Kamiya T, Kawachi N, Watanabe S, Arakawa K and Takahashi T 2020 Imaging of 99m Tc-DMSA and 18 F-FDG in humans using a Si / CdTe Compton camera Phys Med Bio/ 65 05LT01

[97] Yoshida E, Tashima H, Nagatsu K, Tsuji A, Kamada K, Parodi K and Yamaya T 2020 Whole gamma imaging: a new concept of PET combined with Compton imaging Phys. Med. Biol. in press (accepted 29 April 2020)

[98] Toramatsu C, Yoshida E, Wakizaka H, Mohammadi A, Ikoma Y, Tashima H, Nishikido F, Kitagawa A, Karasawa K, Hirano Y and Yamaya T 2018 Washout effect in rabbit brain: in-beam PET measurements using 10C, 11C and 150 ion beams Biomed. Phys. Eng. Express 4035001

[99] Conti M 2010 Why is TOF PET reconstruction a more robust method in the presence of inconsistent data? Phys. Med. Biol. 56 155-68

[100] Vandenberghe S, Daube-Witherspoon M E, Lewitt R M and Karp J S 2006 Fast reconstruction of 3D time-of-flight PET data by axial rebinning and transverse mashing Phys. Med. Biol. 51 1603-21

[101] Ahn S, Cheng L, Shanbhag D D, Qian H, Kaushik S S, Jansen F P and Wiesinger F 2018 Joint estimation of activity and attenuation for PET using pragmatic MR-based prior: application to clinical TOF PET/MR whole-body data for FDG and non-FDG tracers Phys. Med. Biol. 6345006

[102] Defrise M, Rezaei A and Nuyts J 2012 Time-of-flight PET data determine the attenuation 
sinogram up to a constant Phys. Med. Biol. 57 885-99

[103] Rezaei A, Schramm G, Van Laere K and Nuyts J 2019 Estimation of Crystal Timing Properties and Efficiencies for the Improvement of (Joint) Maximum-Likelihood Reconstructions in TOFPET IEEE Trans. Med. Imaging 39 952-63

[104] Watson C C, Hu J and Zhou C 2020 Double Scatter Simulation for More Accurate Image Reconstruction in Positron Emission Tomography IEEE Trans. Radiat. Plasma Med. Sci. 4 57084

[105] Carson R E and Kuo P H 2019 Brain-Dedicated Emission Tomography Systems: A Perspective on Requirements for Clinical Research and Clinical Needs in Brain Imaging IEEE Trans. Radiat. Plasma Med. Sci. 3 254-61

[106] Ren S, Lu Y, Bertolli O, Thielemans K and Carson R E 2019 Event-by-event non-rigid data-driven PET respiratory motion correction methods: comparison of principal component analysis and centroid of distribution Phys. Med. Biol. 64165014

[107] Ilisie V, Giménez-Alventosa V, Moliner L, Sánchez F, González A J, Rodríguez-Álvarez M J and Benlloch J M 2018 Building blocks of a multi-layer PET with time sequence photon interaction discrimination and double Compton camera Nucl. Instruments Methods Phys. Res. Sect. A Accel. Spectrometers, Detect. Assoc. Equip. 895 74-83

[108] Karp J S, Viswanath V, Geagan M J, Muehllehner G, Pantel A R, Parma M J, Perkins A E, Schmall J P, Werner M E and Daube-Witherspoon M E 2020 PennPET Explorer: Design and preliminary performance of a whole-body imager J. Nucl. Med. 61 136-43

[109] Herzog H, Tellmann L, Fulton R, Stangier I, Kops E R, Bente K, Boy C, Hurlemann R and Pietrzyk $\mathrm{U} 2005$ Motion artifact reduction on parametric PET images of neuroreceptor binding J. Nucl. Med. 46 1059-65

[110] Jiao J, Searle G E, Schnabel J A and Gunn R N 2015 Impact of image-based motion correction on dopamine D3/D2 receptor occupancy-comparison of groupwise and frame-by-frame registration approaches EJNMMI Phys. 2 1-15

[111] Bloomfield P M, Spinks T J, Reed J, Schnorr L, Westrip A M, Livieratos L, Fulton R and Jones T 2003 The design and implementation of a motion correction scheme for neurological PET Phys. Med. Biol. 48 959-78

[112] Rahmim A, Dinelle K, Cheng J C, Shilov M A, Segars W P, Lidstone S C, Blinder S, Rousset O G, Vajihollahi H, Tsui B M W, Wong D F and Sossi V 2008 Accurate event-driven motion compensation in high-resolution PET incorporating scattered and random events IEEE Trans. Med. Imaging 27 1018-33

[113] Kyme A Z, Angelis G I, Eisenhuth J, Fulton R R, Zhou V, Hart G, Popovic K, Akhtar M, Ryder W J, Clemens K, Balleine B, Parmar A, Pascali G, Perkins G and Meikle S R 2019 Open-field PET: Simultaneous brain functional imaging and behavioural response measurements in freely moving small animals Neuroimage 188 92-101

[114] Kyme A Z, Zhou V W, Meikle S R and Fulton R R 2008 Real-time 3D motion tracking for small animal brain PET. Phys. Med. Biol. 53

[115] Olesen O V, Paulsen R R, Højgaard L, Roed B and Larsen R 2012 Motion tracking for medical imaging: A nonvisible structured light tracking approach IEEE Trans. Med. Imaging 31 79-87

[116] Liu C, Alessio A M and Kinahan P E 2011 Respiratory motion correction for quantitative PET/CT using all detected events with internal-external motion correlation Med. Phys. 38 2715-23

[117] Rakvongthai $Y$ and El-Fakhri G 2017 Magnetic Resonance-based Motion Correction for Quantitative PET in Simultaneous PET-MR Imaging PET Clin 12 321-7

[118] Kesner A, Schmidtlein C R and Kuntner C 2019 Real-time data-driven motion correction in PET 
Eur. J. Nucl. Med. Mol. Imaging 6 1-6

[119] Blume M, Martinez-Möller A, Keil A, Navab N and Rafecas M 2010 Joint reconstruction of image and motion in gated positron emission tomography IEEE Trans Med Imaging 29 1892906

[120] Rezaei A, Deroose C M, Vahle T, Boada F and Nuyts J 2018 Joint reconstruction of activity and attenuation in time-of-flight PET: A quantitative analysis J. Nucl. Med. 59 1630-5

[121] Kolbitsch C, Neji R, Fenchel M, Schuh A, Mallia A, Marsden P and Schaeffter T 2018 Joint cardiac and respiratory motion estimation for motion-corrected cardiac PET-MR Phys. Med. Biol. 64 15007

[122] Li T, Zhang M, Qi W, Asma E and Qi J 2020 Motion correction of respiratory-gated PET images using deep learning based image registration framework Phys. Med. Biol.

[123] Zanderigo F, Mann J J and Ogden R T 2017 A hybrid deconvolution approach for estimation of in vivo non-displaceable binding for brain PET targets without a reference region PLoS One 12 $1-29$

[124] Gunn R N, Slifstein M, Searle G E and Price J C 2015 Quantitative imaging of protein targets in the human brain with PET Phys. Med. Biol. 60 R363-411

[125] Ceccarini J, Liu H, Van Laere K, Morris E D and Sander C Y 2020 Methods for Quantifying Neurotransmitter Dynamics in the Living Brain With PET Imaging Front. Physiol. 11 1-8

[126] Dutta J, Leahy R and Li Q 2013 Non-local means denoising of dynamic PET images. PLoS One 8 e81390

[127] Rahmim A, Lodge M A, Karakatsanis N A, Panin V Y, Zhou Y, McMillan A, Cho S, Zaidi H, Casey M E and Wahl R L 2019 Dynamic whole-body PET imaging: principles, potentials and applications Eur. J. Nucl. Med. Mol. Imaging 46 501-18

[128] Zanotti-Fregonara P, Chen K, Liow J S, Fujita M and Innis R B 2011 Image-derived input function for brain PET studies: Many challenges and few opportunities J. Cereb. Blood Flow Metab. 31 1986-98

[129] McCluskey S P, Plisson C, Rabiner E A and Howes O 2020 Advances in CNS PET: the state-ofthe-art for new imaging targets for pathophysiology and drug development Eur. J. Nucl. Med. Mol. Imaging 47 451-89

[130] Sharma R and Aboagye E 2011 Development of radiotracers for oncology - The interface with pharmacology Br. J. Pharmacol. 163 1565-85

[131] Leahy R, Boellaard R and Zaidi H 2018 Whole-body parametric PET imaging will replace conventional image-derived PET metrics in clinical oncology Med. Phys. 45 5355-8

[132] Knudsen G M, Ganz M, Appelhoff S, Boellaard R, Bormans G, Carson R E, Catana C, Doudet D, Gee A D, Greve D N, Gunn R N, Halldin C, Herscovitch P, Huang H, Keller S H, Lammertsma A A, Lanzenberger R, Liow J S, Lohith T G, Lubberink M, Lyoo C H, Mann J J, Matheson G J, Nichols T E, Nørgaard M, Ogden T, Parsey R, Pike V W, Price J, Rizzo G, Rosa-Neto P, Schain M, Scott P J $H$, Searle $G$, Slifstein $M$, Suhara $T$, Talbot $P S$, Thomas A, Veronese $M$, Wong D F, Yaqub $M$, Zanderigo F, Zoghbi S and Innis R B 2020 Guidelines for the content and format of PET brain data in publications and archives: A consensus paper J. Cereb. Blood Flow Metab.

[133] Pan L, Cheng C, Haberkorn U and Dimitrakopoulou-Strauss A 2017 Machine learning-based kinetic modeling: a robust and reproducible solution for quantitative analysis of dynamic PET data Phys. Med. Biol. 62 3566-81

[134] Boellaard R, Delgado-Bolton R, Oyen W J G, Giammarile F, Tatsch K, Eschner W, Verzijlbergen F J, Barrington S F, Pike L C, Weber W A, Stroobants S, Delbeke D, Donohoe K J, Holbrook S, Graham M M, Testanera G, Hoekstra O S, Zijlstra J, Visser E, Hoekstra C J, Pruim J, Willemsen A, Arends B, Kotzerke J, Bockisch A, Beyer T, Chiti A, Krause B J, Boellaard R, Hoekstra O S, 
Delgado-Bolton R, Oyen W J G, Visser E, Giammarile F, Tatsch K, Eschner W, Verzijlbergen F J, Barrington S F, Pike L C, Weber W A, Stroobants S, Delbeke D, Donohoe K J, Israel B, Holbrook S, Graham M M, Testanera G and Chiti : A 2015 FDG PET/CT: EANM procedure guidelines for tumour imaging: version 2.0 Eur J Nucl Med Mol Imaging 42 328-54

[135] Kaalep A, Huisman M, Sera T, Vugts D and Boellaard R 2018 Feasibility of PET/CT system performance harmonisation for quantitative multicentre 89Zr studies EJNMMI Phys. 5 1-8

[136] Sunderland J J and Christian P E 2015 Quantitative PET/CT Scanner Performance Characterization Based Upon the Society of Nuclear Medicine and Molecular Imaging Clinical Trials Network Oncology Clinical Simulator Phantom J Nucl Med 56 145-52

[137] Ly J, Minarik D, Edenbrandt L, Wollmer P and Trägårdh E 2019 The use of a proposed updated EARL harmonization of 18F-FDG PET-CT in patients with lymphoma yields significant differences in Deauville score compared with current EARL recommendations EJNMMI Res. 9 65

[138] Namias M and Jeraj R 2019 Patient and scanner-specific variable acquisition times for wholebody PET/CT imaging Phys. Med. Biol. 64205013

[139] Galavis P E, Hollensen C, Jallow N, Paliwal B and Jeraj R 2010 Variability of textural features in FDG PET images due to different acquisition modes and reconstruction parameters Acta Oncol. (Madr). 49 1012-6

[140] Velasquez L, Boellaard R, Kollia G, Hayes W, Hoekstra O, Lammertsma A and Galbraith S 2009 Repeatability of 18F-FDG PET in a multicenter phase I study of patients with advanced gastrointestinal malignancies J Nucl Med $\mathbf{5 0}$ 1646-54

[141] Hatt M, Lee J A, Schmidtlein C R, Naqa I El, Caldwell C, De Bernardi E, Lu W, Das S, Geets X, Gregoire V, Jeraj R, MacManus M P, Mawlawi O R, Nestle U, Pugachev A B, Schöder H, Shepherd T, Spezi E, Visvikis D, Zaidi $H$ and Kirov A S 2017 Classification and evaluation strategies of autosegmentation approaches for PET: Report of AAPM task group No. 211 Med. Phys. 44 e1-42

[142] Bailey D L and Willowson K P 2014 Quantitative SPECT/CT: SPECT joins PET as a quantitative imaging modality Eur. J. Nucl. Med. Mol. Imaging 41

[143] Hudson H M and Larkin R S 1994 Accelerated image reconstruction using ordered subsets of projection data IEEE Trans Med Imag MI-13 601-9

[144] Gholami Y H, Willowson K P, Forwood N J, Harvie R, Hardcastle N, Bromley R, Ryu H J, Yuen S, Howell V M, Kuncic Z and Bailey D L 2018 Comparison of radiobiological parameters for 90Y radionuclide therapy (RNT) and external beam radiotherapy (EBRT) in vitro EJNMMI Phys. $\mathbf{5}$

[145] Häggström I, Schmidtlein C R, Campanella G and Fuchs T J 2019 DeepPET: A deep encoderdecoder network for directly solving the PET image reconstruction inverse problem Med. Image Anal. 54 253-62

[146] Gong K, Wu D, Li Q, Kim K, Yang J, El Fakhri G and Seo Y 2019 EMnet: an unrolled deep neural network for PET image reconstruction Medical Imaging 2019: Physics of Medical Imaging vol 10948, ed H Bosmans, G-H Chen and T Gilat Schmidt (SPIE) p 185

[147] Kim K, Wu D, Gong K, Dutta J, Kim J H, Son Y D, Kim H K, El Fakhri G and Li Q 2018 Penalized PET Reconstruction Using Deep Learning Prior and Local Linear Fitting IEEE Trans. Med. Imaging 37 1478-87

[148] Yang J, Park D, Gullberg G T and Seo Y 2019 Joint correction of attenuation and scatter in image space using deep convolutional neural networks for dedicated brain 18F-FDG PET Phys. Med. Biol. 6475019

[149] Liu C-C and Qi J 2019 Higher SNR PET image prediction using a deep learning model and MRI image Phys. Med. Biol. 64115004 
[150] Song T A, Chowdhury S R, Yang F and Dutta J 2020 PET image super-resolution using generative adversarial networks Neural Networks 125 83-91

[151] Perk T, Bradshaw T, Chen S, Im H, Cho S, Perlman S, Liu G and Jeraj R 2018 Automated classification of benign and malignant lesions in 18F-NaF PET/CT images using machine learning Phys. Med. Biol. 63225019

[152] Cui J, Gong K, Guo N, Wu C, Meng X, Kim K, Zheng K, Wu Z, Fu L, Xu B, Zhu Z, Tian J, Liu H and Li Q 2019 PET image denoising using unsupervised deep learning Eur. J. Nucl. Med. Mol. Imaging 46 2780-9

[153] Lei Y, Dong X, Wang T, Higgins K, Liu T, Curran W J, Mao H, Nye J A and Yang X 2019 Wholebody PET estimation from low count statistics using cycle-consistent generative adversarial networks Phys. Med. Biol. 64215017 